Project No. 10-938

\title{
Modeling Solute Thermokinetics in LiCl-KCl Molten Salt for Nuclear Waste Separation
}

\section{Fuel Cycle R\&D}

Dr. Dane Morgan

In collaboration with: North Carolina State University

Kimberly Gray, Federal POC Guy Fredrickson, Technical POC

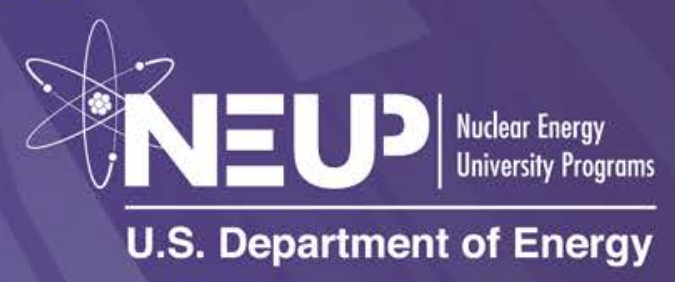




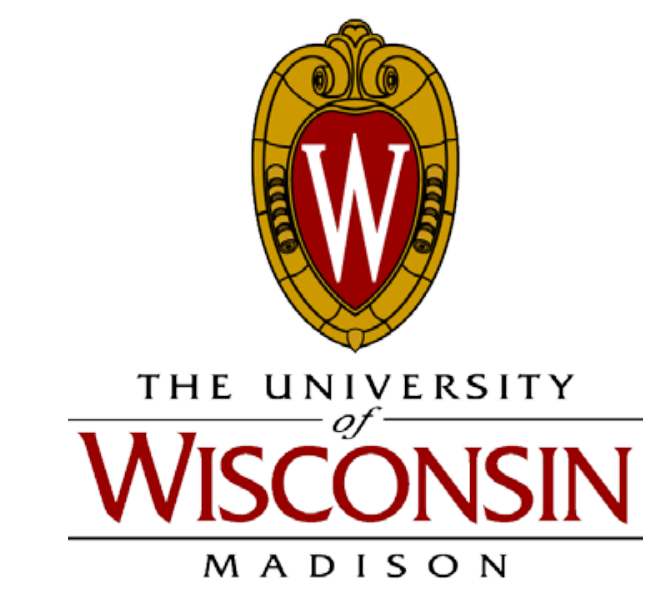

\section{NC STATE UNIVERSITY}

\section{Modeling Solute Thermokinetics in LiCl-KCl Molten Salt for Nuclear Waste Separation (NEUP 10-418)}

Project Investigators: Dane Morgan (University of Wisconsin) and Jacob Eapen (North Carolina State University)

Post-Doctoral Research Associates: Amelia Bengtson, Hyo On Nam, Saumitra Saha, Ridwan Sakidja, Katharina Vörtler (University of Wisconsin) and Brahmananda Chakraborty (North Carolina State University)

Graduate Student: Jin Wang (North Carolina State University)

University of Wisconsin-Madison

NEUP Final Report

Date: September $30^{\text {th }}, 2013$ 


\section{TABLE OF CONTENTS}

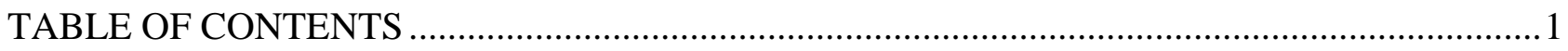

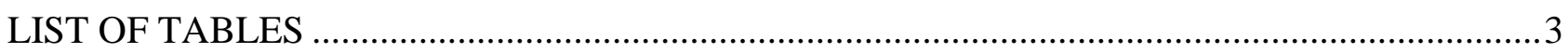

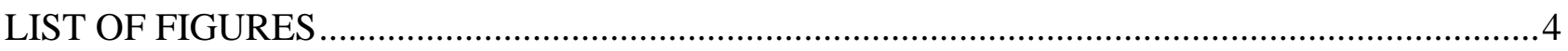

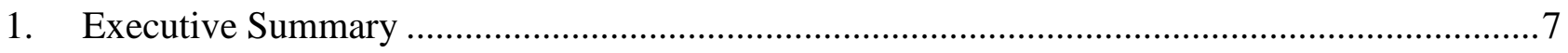

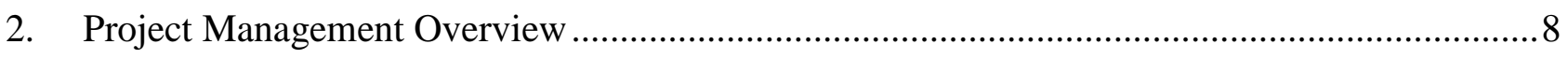

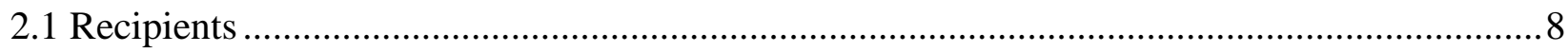

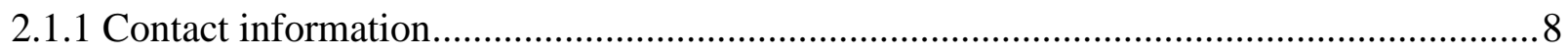

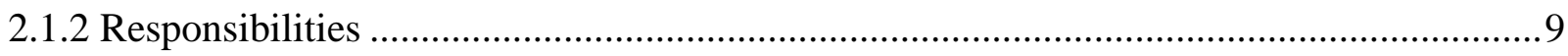

2.3 Project Schedule (Gantt chart) .........................................................................................

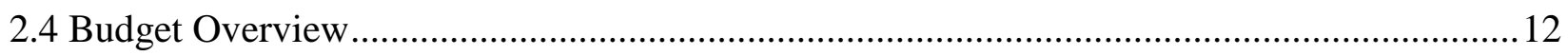

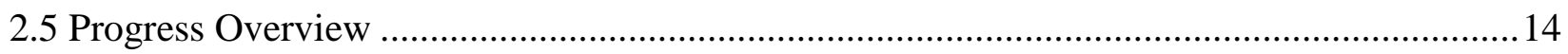

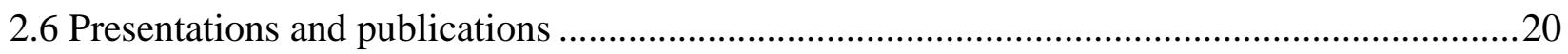

3. Technical Report from North Carolina State University (Task 3) ...........................................21

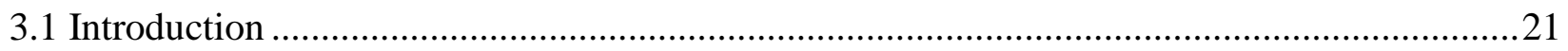

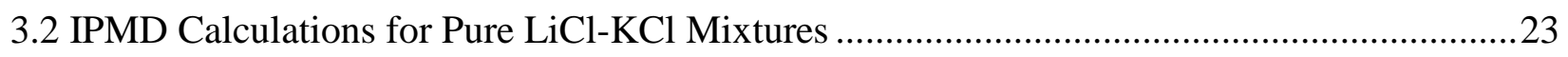

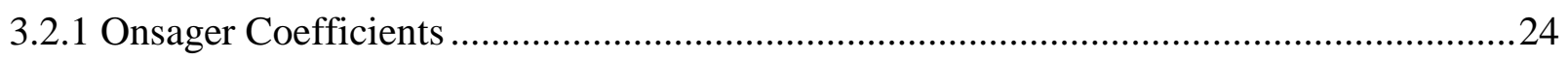

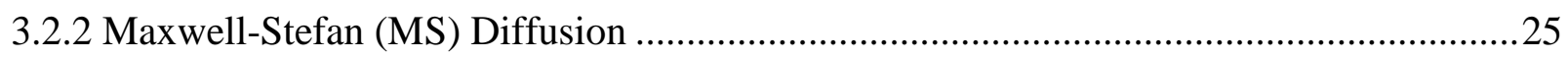

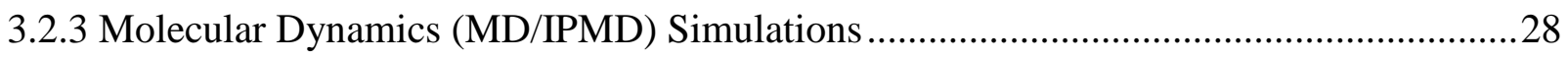

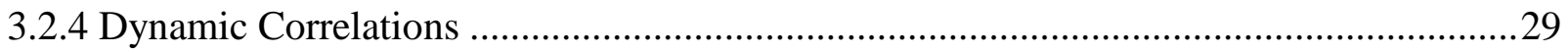

3.2.5 Negative and Divergent Maxwell-Stefan (MS) Diffusivities ...........................................32

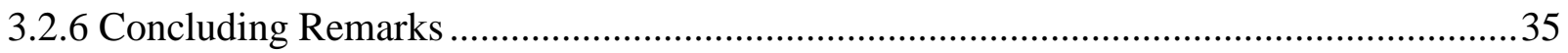

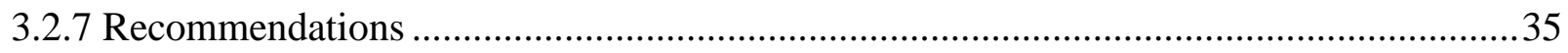

3.3 Free Energy Simulations Development/Analysis .............................................................36

3.3.1 The Two-Phase Thermodynamic (2PT) Method ………………….................................36

3.3.2 Partial Volumes and Mixture Properties .........................................................................4

3.3.3 Molecular Dynamics Simulations ...............................................................................

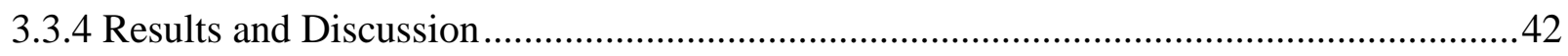

3.3.4.1 Numerical convergence of the fluidicity parameter .......................................................42

3.3.4.2 Benchmarking of 2PT free energy with thermodynamic integration (TI) method ..........43

3.3.4.3 Free energy and entropy change across melting in $\mathrm{KCl}$................................................45

3.3.4.4 Molar free energy and entropy of eutectic LiCl-KCl mixture .........................................4

3.3.5 Concluding Remarks .................................................................................................4

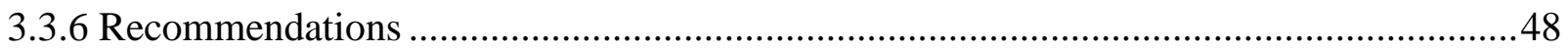


3.4 Prediction of Properties with Sr/La/U solute ...............................................................49

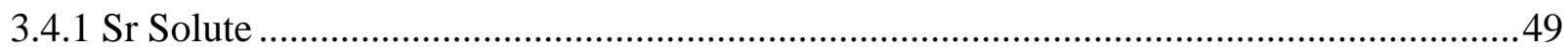

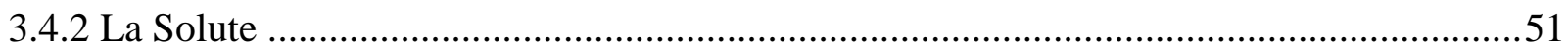

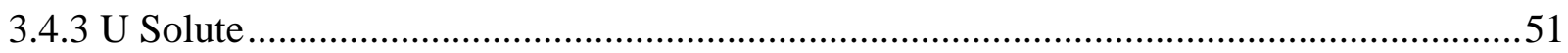

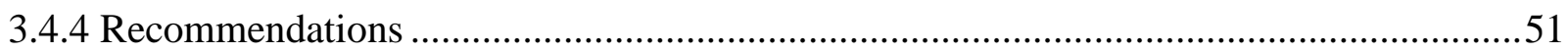

4. Technical Report from the University of Wisconsin-Madison (Task 1 \& 2)..........................52

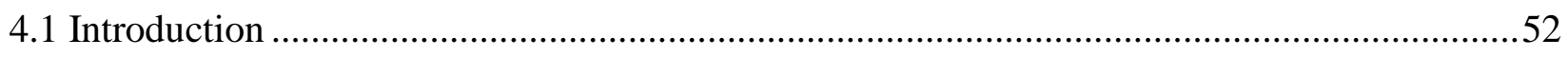

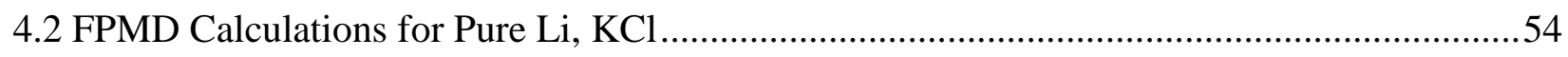

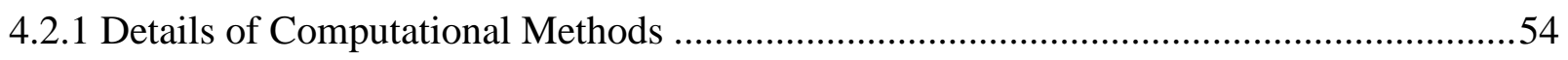

4.2.2 Testing Convergence for FPMD Simulations ...........................................................5

4.2.2.1 Creating converged liquid structures.........................................................................5

4.2.2.2 Testing convergence with simulation time ................................................................58

4.2.2.3 Testing convergence with unit cell size ..................................................................59

4.2.3 Results for Pure LiCl-KCl Salts ........................................................................60

4.2.3.1 Equilibrium volumes, thermal expansion, bulk modulus ...........................................60

4.2.3.2 Self-diffusion coefficients ...........................................................................................64

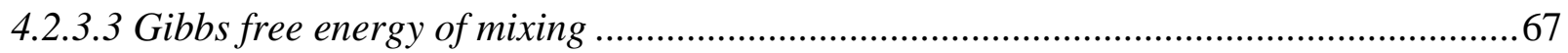

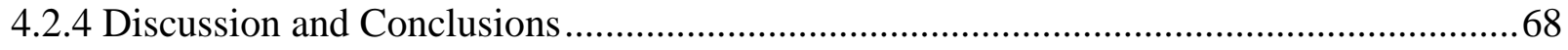

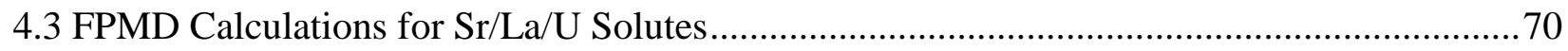

4.3.1 Local Structure Surrounding Solutes in LiCl-KCl ..................................................70

4.3.1.1 Local structure analysis for $\mathrm{Sr} / \mathrm{La} / \mathrm{U}$ solutes.........................................................70

4.3.1.2 Local structure analysis for other solutes $(\mathrm{Eu} / \mathrm{Nd} / \mathrm{Ce} / \mathrm{Zr})$........................................71

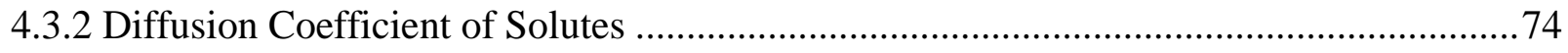

4.3.3 Standard Redox Potential for Solutes in Molten LiCl-KCl Eutectic Salt ........................75

4.4 Interatomic Potential Development for Sr/La/U Solutes ................................................79

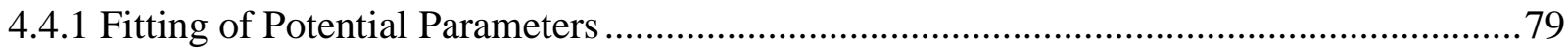

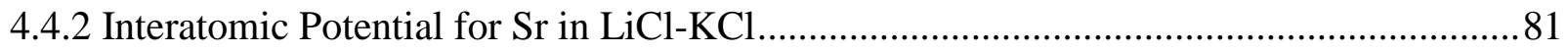

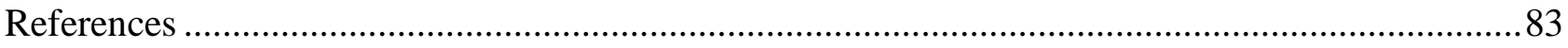




\section{LIST OF TABLES}

Table 3.1. The 2PT results of Sr solute system at $773 \mathrm{~K}$ and $0 \mathrm{~Pa}$. A total of 990 ions are used with

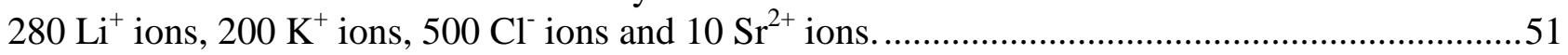

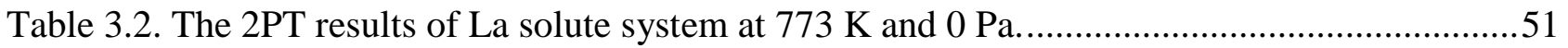

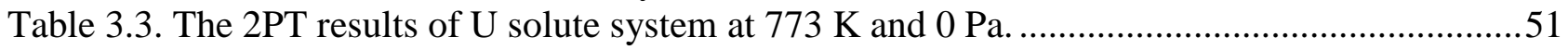

Table 4.1. Equilibrium volume $\left(\mathrm{V}_{0}\right)$ from FPMD with 216 atoms. Simulations were run at multiple volumes for the NVT ensemble. $\mathrm{V}$, $\mathrm{P}$ were fit to a Murnaghan equation of state to obtain $\mathrm{V}_{0}$ (Experimental $\mathrm{V}_{0}$ : Janz et al. (1979) [116] (extrapolated for some temperatures)).....

Table 4.2. Coefficient of thermal expansion, $\alpha_{V}$ at $1096 \mathrm{~K}$ from FPMD with 216 atoms.

Table 4.3. Self-diffusion coefficients for $\mathrm{Li}, \mathrm{Cl}$ and $\mathrm{K}$ in $\mathrm{LiCl}, \mathrm{KCl}$ and eutectic LiCl-KCl. Mean squared displacement (MSD) were calculated at the volume closest to equilibrium volume from the set of FPMD simulations for the EOS analysis. Each simulation was performed with the NVT ensemble and 216 atoms for 6 ps. MSD analysis started after the first 200 time steps (for equilibration). The remaining time steps were divided into 4 equal blocks. The MSD vs. time curve was fit for all but the initial 25 steps of each block in order to avoid the quadratic region.

Table 4.4. First-peak radius and height, and first-shell coordination numbers for solutes and F pairs in LiCl-KCl.

Table 4.5. Estimated diffusion coefficients of solutes in the $\mathrm{LiCl}-\mathrm{KCl}$ at $773 \mathrm{~K}$ from FPMD simulations. Error ranges represent one sigma standard error of the mean from 9 simulations. .75 Table 4.6 Calculated redox potential of several redox couples in molten LiCl-KCl salt at 773K with the predicted redox potential from the thermodynamic database [133]. .78

Table 4.7. Parameters for Tosi-Fumi potential for the LiCl-KCl system with Sr 


\section{LIST OF FIGURES}

Figure 2.1. Schedule and timeline of project (from proposal). There are three main tasks for this project (black bars), each with individual subcomponents (blue bars). Progress on all milestones and tasks were reported using the language of this Gantt chart. Dark shading denotes the percent of the task that is complete; light shading indicates the amount of the task remaining.

Figure 3.1. Short-ranged BHM interactions for $\mathrm{LiCl}-\mathrm{KCl}$ mixture. (Inset) The interactions for the

$\mathrm{Li}$ group ( $\mathrm{Li} \mathrm{Li}, \mathrm{Li}-\mathrm{K}$ and $\mathrm{Li}-\mathrm{Cl}$ ) on a magnified scale.

Figure 3.2. (a) Temporal variation of diffusive flux correlation, $\Psi(t)$ at $773 \mathrm{~K}$. Insets show the normalized diffusive flux correlations for $\mathrm{Li}-\mathrm{Li}, \mathrm{Li}-\mathrm{Cl}$ and $\mathrm{K}-\mathrm{K}$; (b) Temporal variation of diffusive flux correlation, $\Psi(t)$ at $1200 \mathrm{~K}$. Insets show the normalized diffusive flux correlations for $\mathrm{Li}-\mathrm{Li}, \mathrm{Li}-\mathrm{Cl}$ and $\mathrm{K}-\mathrm{K}$.

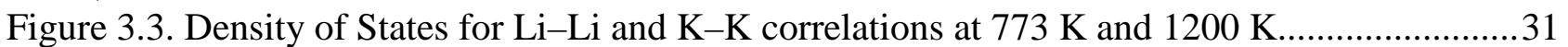

Figure 3.4. Onsager coefficients ( $\Lambda$ matrix elements) at eutectic composition for different temperatures. The broken straight lines serve as guide to the eye.

Figure 3.5. MS diffusivities for different temperatures at eutectic composition. (Inset) $\mathrm{D}_{\mathrm{Li}-\mathrm{Cl}}$ and

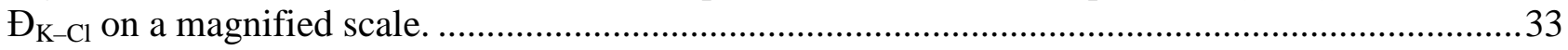

Figure 3.6. Entropy constraint [Eqn. (3.2.20)] showing a non-negative entropy production rate......34 Figure 3.7. MS diffusivities for different compositions at 1043 K. (Inset) Experimental MS diffusivities in an ion exchange membrane (data from [62]).

Figure 3.8. Relative error in reconstructing the fluidicity parameter for the $\mathrm{K}^{+}$ions in molten $\mathrm{KCl} .43$ Figure 3.9. Comparison of absolute (molar) free energy of molten $\mathrm{KCl}$ from the 2PT and TI [18] methods at a temperature of $1100 \mathrm{~K}$ and a density of $20 \mathrm{~nm}^{-3}$....

Figure 3.10. Molar free energy change across melting of $\mathrm{KCl}$ with the 2PT method. (Inset) Entropy jump from 2PT and comparison to NIST standard entropy data (open circles) [78].

Figure 3.11. Density of states (DoS): total (left column), solid-like (HO, middle column), and gaslike (HS, right column) for $\mathrm{Li}^{+}$(top row) and $\mathrm{K}^{+}$ions (bottom row) at different temperatures ( $773 \mathrm{~K}$ to $1300 \mathrm{~K}$ ). The DoS plots for different temperatures - $773 \mathrm{~K}, 850 \mathrm{~K}, 950 \mathrm{~K}, 1043 \mathrm{~K}, 1100 \mathrm{~K}, 1150$ $\mathrm{K}, 1200 \mathrm{~K}, 1300 \mathrm{~K}$ ) - are off-set by 40 ps for visual clarity....

Figure 3.12. Free energy/entropy for $\mathrm{LiCl}-\mathrm{KCl}$ mixture at eutectic composition $\left(x_{\mathrm{KCL}}=0.42\right) \ldots \ldots . .47$ Figure 3.13. Mass-weighted velocity autocorrelation (JACF) for the Sr solute system. (Inset) JACF for $\mathrm{Sr}^{2+}$ ions. A total of 990 ions are used with $280 \mathrm{Li}^{+}$ions, $200 \mathrm{~K}^{+}$ions, $500 \mathrm{Cl}^{-}$ions and $10 \mathrm{Sr}^{2+}$ ions.

Figure 3.14. The density of states (DoS) of the Sr solute system at $773 \mathrm{~K}$ and 0 pressure. The total DoS $(G)$ is shown in black, the hard sphere phase $(H S)$ in red and the harmonic oscillator (Solid) in blue. A total of 990 ions are used with $280 \mathrm{Li}^{+}$ions, $200 \mathrm{~K}^{+}$ions, $500 \mathrm{Cl}^{-}$ions and $10 \mathrm{Sr}^{2+}$ ions.....50 Figure 4.1. Comparison of FPMD computational choices at $1096 \mathrm{~K}$ in VASP. All simulations were run with 64 atoms. PBE, PBEsol and LDA are different choices controlling the exchange correlation within a density functional theory calculation. EXP is experimental data from Janz et al. [116] either at $1096 \mathrm{~K}$ or extrapolated to $1096 \mathrm{~K}$.

Figure 4.2. Structural parameters for the 64-atom $\mathrm{LiCl}$ structure. (a) FPMD calculations started from solid, crystalline structure and run at $1000 \mathrm{~K}$ for $18 \mathrm{ps}$. Long-range order is not lost, suggesting the material is not molten. (b) Classical IPMD simulations using the LAMMPS code run for 100 ps. (c) FPMD calculations starting from liquid structure equilibrated in LAMMPS from part (b) run for $6 \mathrm{ps}$. .58

Figure 4.3. Convergence of (a) temperature (b) pressure (c) total energy and (d) self-diffusion coefficients with simulation time. Simulations were run for 6, 12, 20, 50, 100, and 200 ps. All simulations were run as NVT with IPMD (using LAMMPS) for LiCl with 64 atoms at $1096 \mathrm{~K}$ with 
a timestep of 2 fs. All simulations started from the same converged IPMD simulation that would be used to start a VASP (FPMD) simulation. Desired simulation times for VASP are 5-10 ps. Errors were calculated from the autocovariance function. Averaging started after 0.5 ps so as to use only the equilibrated region.

Figure 4.4. Convergence of (a) volume (b) pressure (c) energy and (d) temperature with unit cell size in LiCl. Simulations were run with IPMD with the method outlined in the Computational methods section (NVE, crude barostat, NPT $(\mathrm{P}=0)$ for 100,000 steps, NVT for 100,000 steps). Volumes and errors in (a) come from NPT simulation analyzed over last $90 \%$ of data set. (b, c, d) results are from NVT simulations where the last $90 \%$ of simulation was analyzed. Errors are from analytical method.

Figure 4.5. Pressure-volume curves from VASP FPMD simulations with the NVT ensemble for of

(a) $\mathrm{LiCl}$ (b) $\mathrm{KCl}$ and (c) $\mathrm{LiCl}-\mathrm{KCl}$ with the eutectic composition. Experimental volumes are from (*extrapolated from) Janz et al. [116].

Figure 4.6. Volume vs. temperature for (a) $\mathrm{LiCl}$ (b) $\mathrm{KCl}$ and (c) $\mathrm{LiCl}-\mathrm{KCl}$ with the eutectic composition at multiple temperatures. $\mathrm{LiCl}-\mathrm{KCl}$ volumes are plotted per $\mathrm{Cl}$ atom. EXP1 = Janz et al. (1979) [116]. EXP2 = Van Artsdalen and Yaffe (1955) [120], EXP3 = Ito et al. (2001) [121].

Dashed lines indicate the experimental error between the experimental datasets.

Figure 4.7. Bulk modulus vs. temperature for the LiCl-KCl system.

Figure 4.8. Self-diffusion coefficients from FPMD simulations for (a) $\mathrm{Li}$ in $\mathrm{LiCl}$ (b) $\mathrm{Cl}$ in $\mathrm{LiCl}$ (c) $\mathrm{K}$ in $\mathrm{KCl}$ and (d) $\mathrm{Cl}$ in $\mathrm{KCl}$. Experimental values for $\mathrm{LiCl}$ are from Lenke et al. [122] and Janz et al. [116] and references within Lantelme and Turq [88] and Morgan and Madden [123]. Computational IPMD values for LiCl are taken from Lantelme and Turq [88], Caccamo and Dixon [89], and Morgan and Madden [123] and references within. Experimental values for $\mathrm{KCl}$ are from Morgan and Madden [123] and Janz [124] and references within. Computational IPMD values for KCl are from Lantelme and Turq [88], Caccamo and Dixon [89], Hazebroucq et al. [98], and Morgan and Madden [123]. All simulations were run with FPMD in VASP at the volume closest to equilibrium (LiCl: $23.76 \AA^{3}$ /atom at $1096 \mathrm{~K}, 23.15 \AA^{3}$ /atom at $1000 \mathrm{~K}, 22.55 \AA^{3}$ /atom at $900 \mathrm{~K}$; KCl: 44.74 $\AA^{3}$ /atom at $1200 \mathrm{~K}, 42.88 \AA^{3}$ /atom at $1096 \mathrm{~K}$ ) with the NVT ensemble and 216 atoms for 6 ps. Analysis started after the first 200 time steps (for equilibration). The remaining time steps were divided into 4 equal blocks. The MSD vs. time curve was fit for all but the initial 25 steps of each block in order to avoid the quadratic region.

Figure 4.9. Self-diffusion coefficients from FPMD simulations for (a) Li, (b) $\mathrm{Cl}$ and (c) $\mathrm{K}$ in eutectic LiCl-KCl. All simulations were run with FPMD in VASP at the volume closest to equilibrium (31.26 $\AA^{3}$ /atom at $1096 \mathrm{~K}, 31.26 \AA^{3}$ /atom at $1000 \mathrm{~K}$, and $29.31 \AA^{3}$ /atom at $\left.800 \mathrm{~K}\right)$ with the NVT ensemble and 216 atoms for 6 ps. Analysis started after the first 200 time steps (for equilibration). The remaining time steps were divided into 4 equal blocks. The MSD vs. time curve was fit for all but the initial 25 steps of each block in order to avoid the quadratic region. Results are plotted with IPMD simulations from the literature: Lantelme and Turq [88] and Caccamo and Dixon [89]

Figure 4.10. Gibbs free energy at $1096 \mathrm{~K}$. The fit to experimental data (dashed line) comes from the FTsalt - FACT salt database (FactSage) [125].

Figure 4.11. Snapshots of molecular dynamics simulation trajectory, (a) FPMD simulation of the $\mathrm{LiCl}-\mathrm{KCl}$ with $\mathrm{SrCl}_{2}$ showing bonding between $\mathrm{Sr}$ and $\mathrm{Cl}$ ions; (b) local structure of La from the FPMD simulation of the $\mathrm{LiCl}-\mathrm{KCl}$ with $\mathrm{LaCl}_{3}$; (c) local structure of $\mathrm{U}$ from the FPMD simulation of the $\mathrm{LiCl}-\mathrm{KCl}$ with $\mathrm{UCl}_{3}$; (d) local structure of $\mathrm{U}$ from the FPMD simulation of the $\mathrm{LiCl}-\mathrm{KCl}$ with $\mathrm{UCl}_{4}$ .70

Figure 4.12. Radial distribution functions (RDF) of the $\mathrm{LiCl}-\mathrm{KCl}$ (64 atoms) with (a) $\mathrm{SrCl}_{2}$; (b) $\mathrm{LaCl}_{3}$; (c) $\mathrm{UCl}_{3}$; (d) $\mathrm{UCl}_{4}$ between each solute and $\mathrm{Cl}$ ions. 
Figure 4.13. Snapshots of molecular dynamics simulation trajectory, (a) FPMD simulation of the $\mathrm{LiCl}-\mathrm{KCl}$ with $\mathrm{EuCl}_{3}$ showing bonding between Eu and $\mathrm{Cl}$ ions; (b) local structure of $\mathrm{Nd}$ from the FPMD simulation of the $\mathrm{LiCl}-\mathrm{KCl}$ with $\mathrm{NdCl}_{3}$; (c) local structure of Ce from the FPMD simulation of the $\mathrm{LiCl}-\mathrm{KCl}$ with $\mathrm{CeCl}_{3}$; and (d) local structure of $\mathrm{Zr}$ from the FPMD simulation of the LiCl$\mathrm{KCl}$ with $\mathrm{ZrCl}_{4}$

Figure 4.14 Radial distribution functions (RDF) of the LiCl-KCl with (a) $\mathrm{EuCl}_{3}$; (b) $\mathrm{CeCl}_{3}$; (c) $\mathrm{NdCl}_{3}$; (d) $\mathrm{ZrCl}_{4}$ between each solute and $\mathrm{Cl}$ ions. 73 Figure 4.15. Estimated diffusion coefficients of solutes in the $\mathrm{LiCl}-\mathrm{KCl}$ at $773 \mathrm{~K}$. Square marks are $2+$, circles are 3+, and triangles are 4+ ions. Experimental diffusion coefficients (diamonds) are also shown for comparison. Error bars represent one sigma standard error of the mean from 9 simulations. .74

Figure 4.16. Comparison of diffusion coefficient of $\mathrm{La}\left(3^{+}\right)$from FPMD (Red rectangular with standard error) with the experimental measurements [129] ...................................................75 Figure 4.17 Comparison of calculated redox potential of several redox couples in molten LiCl-KCl salt at 773K by FPMD (y-axis) with the (a) experimental redox potential and the (b) predicted redox potential from the thermodynamic database for pure solid form of chlorides [133]. Red dashed lines are showing $\pm 0.5 \mathrm{~V}$ range from the guide line. The error bars represent the two sigma standard error of the mean. .77

Figure 4.18. Comparison between Cartesian force components on individual $\mathrm{K}$ atoms (unit is $\mathrm{meV} / \AA$ ).

Figure 4.19. Comparison between Cartesian force components on individual $\mathrm{Sr}$ atoms (unit is $\mathrm{meV} / \AA$ ). 80

Figure 4.20. Comparison of RDFs of Sr-X showing a reasonable agreement between the results from the IPMD (solid line) and the FPMD (dashed line)

Figure 4.21. Comparison of estimated diffusion coefficients from IPMD (squares) and the FPMD (circles). The error represents the one sigma standard error .82 


\section{Executive Summary}

This project has developed first-principles based molecular modeling and simulation approaches to predict fundamental thermokinetic properties of dissolved actinides and fission products in molten salts. Present nuclear waste separation methods are controlled by solute and salt properties that are difficult and expensive to measure experimentally for the wide range of relevant salt mixtures. The simulation approaches developed in this work can provide essential data from first-principles, thereby reducing the number of required experiments. More broadly, the modeling is of value for interpreting experimental results, guiding new experiments, validating analytical models, and for optimizing waste separation by enabling rapid and inexpensive exploration of new salt configurations and operating conditions.

The properties predicted with the modeling are density, which is used to assess the amount of dissolved material in the salt mixtures, diffusion, which can control rates of material transport during separation, and solute activity, which determines total solubility and reduction potentials used during electrorefining. The work has focused on $\mathrm{LiCl}-\mathrm{KCl}$ salts, which are used for treating spent EBR-II fuel. Studies were performed using $\mathrm{LiCl}, \mathrm{KCl}$, and $\mathrm{LiCl}-\mathrm{KCl}$ eutectic composition (58 mol\% $\mathrm{LiCl}, 42 \mathrm{~mol} \% \mathrm{KCl}$ ) salts. The work focused on $\mathrm{La}, \mathrm{Sr}$, and $\mathrm{U}$ impurities (defined as solutes in this document), which are chosen to include the important distinct categories of lanthanides, alkali earths, and actinides, respectively.

The modeling methods use first-principles and first-principles derived interatomic potential based simulations, primarily using molecular dynamics. Results were validated against existing literature, including some data developed in a parallel ongoing experimental effort at the lead-PIs institution, UW Madison. Although this work is focused on a particular set of systems, the approaches and tools developed will enable prediction of separations relevant salt and solute properties for a wide variety of mixtures.

Objective: Develop and validate molecular modeling approaches to predict density, species diffusion, and solute activity (through free energy) for $\mathrm{La}, \mathrm{Sr}$, and $\mathrm{U}$ in $\mathrm{LiCl}-\mathrm{KCl}$ eutectic salts at different solute concentrations $(0-5 \mathrm{~mol} \%)$ and temperatures $\left(400^{\circ} \mathrm{C}-600^{\circ} \mathrm{C}\right)$.

The work consists of 3 main tasks

- Task 1: First-principles molecular dynamics (FPMD) modeling of salt-solute thermokinetics

- Task 2: Interatomic potential development

- Task 3: Interatomic potential molecular dynamics (IPMD) modeling of salt-solute thermokinetics. 


\section{Project Management Overview}

\subsection{Recipients}

\subsubsection{Contact information}

University of Wisconsin-Madison, 1509 University Ave., Department of Materials Science and Engineering, Madison, WI, 53706

North Carolina State University, 1114 Burlington Engineering Labs, Nuclear Engineering Department, Raleigh, NC, 27695

\section{Principle Investigators}

\begin{tabular}{|l|l|l|l|}
\hline Name & Contact Information & Title & Location \\
\hline Dane Morgan & $\begin{array}{l}\text { 608.265.5879 } \\
\text { ddmorgan@wisc.edu }\end{array}$ & $\begin{array}{l}\text { Associate Professor, } \\
\text { Lead PI }\end{array}$ & $\begin{array}{l}\text { University of Wisconsin-Madison, } \\
\text { Department of Materials Science } \\
\text { and Engineering }\end{array}$ \\
\hline Jacob Eapen & $\begin{array}{l}\text { 919.515.5952 } \\
\text { jacob.eapen@ncsu.edu }\end{array}$ & $\begin{array}{l}\text { Assistant Professor, } \\
\text { co- PI }\end{array}$ & $\begin{array}{l}\text { North Carolina State University, } \\
\text { Department of Nuclear } \\
\text { Engineering }\end{array}$ \\
\hline
\end{tabular}

Postdocs and Graduate Students

\begin{tabular}{|l|l|l|l|}
\hline Name & Contact Information & Title & Location \\
\hline $\begin{array}{l}\text { Amelia (Amy) } \\
\text { Bengtson }\end{array}$ & akbengtson@wisc.edu & $\begin{array}{l}\text { Postdoctoral Research } \\
\text { Associate }\end{array}$ & University of Wisconsin-Madison \\
\hline Saumitra Saha & ssaha3@wisc.edu & $\begin{array}{l}\text { Postdoctoral Research } \\
\text { Associate }\end{array}$ & University of Wisconsin-Madison \\
\hline Hyo On Nam & hnam9@wisc.edu & $\begin{array}{l}\text { Postdoctoral Research } \\
\text { Associate }\end{array}$ & University of Wisconsin-Madison \\
\hline Ridwan Sakidja & rsakidja@facstaff.wisc.edu & Assistant Scientist & University of Wisconsin-Madison \\
\hline Katharina Vörtler & vörtler@wisc.edu & $\begin{array}{l}\text { Postdoctoral Research } \\
\text { Associate }\end{array}$ & University of Wisconsin-Madison \\
\hline $\begin{array}{l}\text { Brahmananda } \\
\text { Chakraborty }\end{array}$ & bchakra@ncsu.edu & $\begin{array}{l}\text { Postdoctoral Research } \\
\text { Associate }\end{array}$ & North Carolina State University \\
\hline Jin Wang & jwang22@ncsu.edu & $\begin{array}{l}\text { Third Year Graduate } \\
\text { Student (Major: } \\
\text { Nuclear Engineering) }\end{array}$ & North Carolina State University \\
\hline
\end{tabular}




\subsubsection{Responsibilities}

\begin{tabular}{|l|l|}
\hline Name & $\begin{array}{l}\text { Primary } \\
\text { Responsibilities }\end{array}$ \\
\hline $\begin{array}{l}\text { Dane } \\
\text { Morgan* }\end{array}$ & $\begin{array}{l}\text { Task 1, Task 2 } \\
\text { Coordinates overall effort and is point of contact for DOE }\end{array}$ \\
\hline Jacob Eapen & Task 3 \\
\hline
\end{tabular}

\begin{tabular}{|l|l|l|}
\hline Name & $\begin{array}{l}\text { Primary } \\
\text { Responsibilities }\end{array}$ & Tasks for Project \\
\hline $\begin{array}{l}\text { Amelia (Amy) } \\
\text { Bengtson }\end{array}$ & Task 1 \& 2 & $\begin{array}{l}\text { She worked on FPMD calculations for Task 1\& 2 and finished } \\
\text { calculations on pure LiCl, KCl, and LiCl-KCl. }\end{array}$ \\
\hline $\begin{array}{l}\text { Saumitra } \\
\text { Saha }\end{array}$ & $\begin{array}{l}\text { different molten salt } \\
\text { system }\end{array}$ & $\begin{array}{l}\text { Saha was not funded from the work and played a supporting } \\
\text { role. He worked on FPMD calculations in a different molten } \\
\text { salt system. }\end{array}$ \\
\hline $\begin{array}{l}\text { Hyo On } \\
\text { Nam }\end{array}$ & Task 1 \& 2 & $\begin{array}{l}\text { Nam continued first-principles work when Bengtson, Saumitra, } \\
\text { and Sakidja departed. He has been the lead researcher and } \\
\text { written the bulk of reports in the final 9 months of the project. }\end{array}$ \\
\hline $\begin{array}{l}\text { Ridwan } \\
\text { Sakidja }\end{array}$ & Task 2 & $\begin{array}{l}\text { Sakidja worked on interatomic potential development for Sr in } \\
\text { LiCl-KCl. }\end{array}$ \\
\hline $\begin{array}{l}\text { Katharina } \\
\text { Vörtler }\end{array}$ & $\begin{array}{l}\text { Primary focus on } \\
\text { different molten salt } \\
\text { system; Some effort } \\
\text { on Tasks 1 \& 2 }\end{array}$ & $\begin{array}{l}\text { Vörtler worked on FPMD calculations primarily in a different } \\
\text { molten salt system, but assisted with some studies for this } \\
\text { project. Nam and Vörtler communicated regularly about FPMD } \\
\text { in molten salts. }\end{array}$ \\
\hline $\begin{array}{l}\text { Brahmananda } \\
\text { Chakraborty }\end{array}$ & Task 3 & $\begin{array}{l}\text { Chakraborty worked on the development and analysis of } \\
\text { multicomponent diffusion and free energy analysis in salt } \\
\text { mixtures with solutes. }\end{array}$ \\
\hline $\begin{array}{l}\text { Jin } \\
\text { Wang }\end{array}$ & Task 3 & $\begin{array}{l}\text { Wang worked on development and analysis of free energy } \\
\text { methods suitable for salt mixtures with solutes. }\end{array}$ \\
\hline
\end{tabular}

The large group met monthly via skype to discuss results, technical details, concerns, and future plans. Members at the same institution communicated regularly. 


\subsection{Resources Overview}

Commercial Codes used:

- VASP-MD (Vienna Ab-Initio Simulation Package): First-principles molecular dynamics (FPMD) simulations that use density functional theory to describe atomic interactions. FPMD calculations are considered more accurate than IPMD as the interactions of electrons are explicitly modeled through density functional theory.

- LAMMPS: Interatomic potential molecular dynamics (IPMD) code that uses empirically fit interatomic potentials to describe the interactions between atoms. Used to create starting melt $(\mathrm{Li}, \mathrm{K}) \mathrm{Cl}$ structures for FPMD calculations.

- DL-POLY-4: IPMD code. DL_POLY is a general purpose classical molecular dynamics simulation software developed at Daresbury Laboratory by I.T. Todorov and W. Smith. It has several interatomic potentials and is capable of handling complex salts with appropriate modifications. Additional multi-component routines needed are diffusion calculations, which entail code modification (in Fortran 90).

- $\quad$ POTFIT, a free code that fits potentials from ab-initio data.

\section{Routines:}

We have written scripts in python to analyze and extract energy and diffusion data from VASP and LAMMPS. Some routines have been developed in Fortran 90 and incorporated in MD package to compute multi-component diffusion coefficients. Free energy calculations are performed with inhouse codes which have been tested and benchmarked.

High-performance computing clusters:

- Local clusters at the University of Wisconsin-Madison (UW-Madison)

- NSF supercomputing resources: Teragrid Ranger/Stampede cluster (UW-Madison)

- Idaho National Lab HPC (UW-Madison)

- NC State High Performance Computing Center (HPC) (NCSU) 


\subsection{Project Schedule (Gantt chart)}

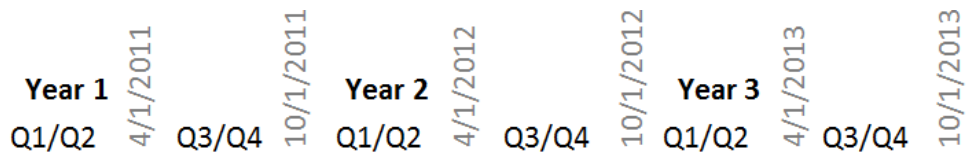

TASK 1: FPMD MODELING

(a) FPMD calculations for pure $\mathrm{Li}, \mathrm{KCl}$

(b) FPMD calculations for Sr solute

(c) FPMD calculations for La solute

(d) FPMD calculations for $U$ solute

TASK 2: INTERATOMIC POTENTIAL DEVELOPMENT

(a) Development of potential for $\mathrm{Sr}$

(b) Development of potential for La

(c) Development of potential for $U$

TASK 3: IPMD MODELING

(a) IPMD calculations for pure $\mathrm{Li}, \mathrm{KCl}$ (validate FPMD)

(b) Free energy simulations development/analysis

(c) Prediction of properties with Sr solute

(d) Prediction of properties with La solute

(e) Prediction of properties with $U$ solute
$99 \%$ complete

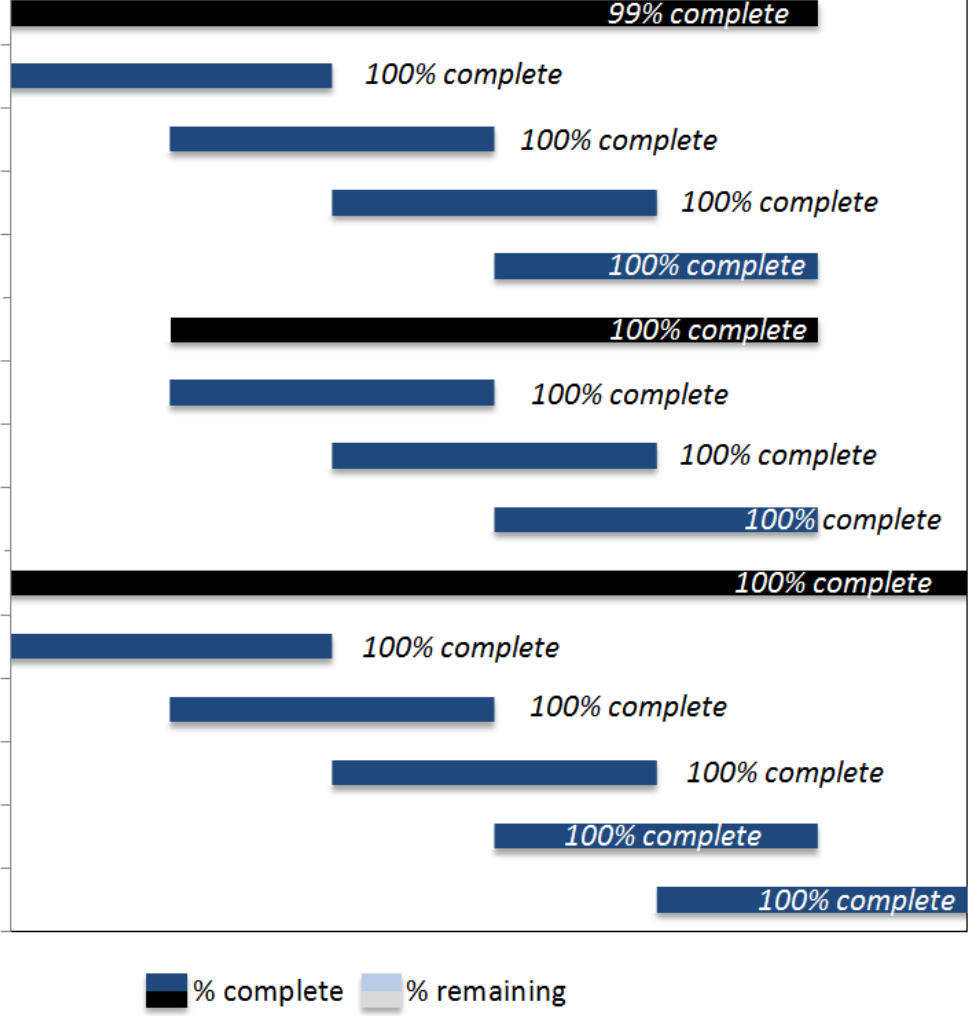

Figure 2.1. Schedule and timeline of project (from proposal). There are three main tasks for this project (black bars), each with individual subcomponents (blue bars). Progress on all milestones and tasks were reported using the language of this Gantt chart. Dark shading denotes the percent of the task that is complete; light shading indicates the amount of the task remaining. 


\subsection{Budget Overview}

FY 2011

\begin{tabular}{|c|c|c|c|c|c|}
\hline \multirow{4}{*}{$\begin{array}{r}\text { Total Approved } \\
\text { Total Actual Expenses } \\
\text { Total Under Budget }\end{array}$} & Q1 & Q2 & Q3 & Q4 & Y1 Totals \\
\hline & $\$ 54,007.25$ & $\$ 54,007.25$ & $\$ 54,007.25$ & $\$ 54,007.25$ & $\$ 216,029.00$ \\
\hline & $\$ 1,729.27$ & $\$ 28,382.81$ & $\$ 29,453.98$ & $\$ 43,913.08$ & $\$ 103,479.14$ \\
\hline & $\$ 52,277.98$ & $\$ 25,624.44$ & $\$ 24,553.27$ & $\$ 10,094.17$ & $\$ 112,549.86$ \\
\hline \multirow{3}{*}{$\begin{array}{l}\text { UW Expected Amount } \\
\text { UW Actual Expenses } \\
\text { UW Under Budget }\end{array}$} & $\$ 29,894.29$ & $\$ 29,894.29$ & $\$ 29,894.29$ & $\$ 29,894.29$ & $\$ 119,577.16$ \\
\hline & $\$ 1,729.27$ & $\$ 21,352.42$ & $\$ 19,012.98$ & $\$ 20,543.08$ & $\$ 62,637.75$ \\
\hline & $\$ 28,165.02$ & $\$ 8,541.87$ & $\$ 10,881.31$ & $\$ 9,351.21$ & $\$ 56,939.41$ \\
\hline \multirow{3}{*}{$\begin{array}{l}\text { NCSU Expected Amount } \\
\text { NCSU Actual Expenses } \\
\text { NCSU Under Budget }\end{array}$} & $\$ 24,112.96$ & $\$ 24,112.96$ & $\$ 24,112.96$ & $\$ 24,112.96$ & $\$ 96,451.84$ \\
\hline & $\$ 0.00$ & $\$ 7,030.39$ & $\$ 10,441.00$ & $\$ 23,370.00$ & $\$ 40,841.39$ \\
\hline & $\$ 24,112.96$ & $\$ 17,082.57$ & $\$ 13,671.96$ & $\$ 742.96$ & $\$ 55,610.45$ \\
\hline
\end{tabular}

FY 2012

\begin{tabular}{|c|c|c|c|c|c|}
\hline \multirow{3}{*}{$\begin{array}{r}\text { Total Approved } \\
\text { Total Actual Expenses }\end{array}$} & Q1 & Q2 & Q3 & Q4 & Y2 Totals \\
\hline & $\$ 50,069.06$ & $\$ 50,069.06$ & $\$ 50,069.06$ & $\$ 50,069.06$ & $\$ 200,276.23$ \\
\hline & $\$ 50,355.24$ & $\$ 54,941.99$ & $\$ 102,009.79$ & $\$ 79,545.57$ & $\$ 286,852.59$ \\
\hline \multirow{2}{*}{$\begin{array}{r}\text { Total Over/Under Budget for } \\
\text { Quarter } \\
\text { Total Under Budget }\end{array}$} & $(\$ 286.18)$ & $(\$ 4,872.93)$ & $(\$ 51,940.73)$ & $(\$ 29,476.51)$ & $(\$ 86,576.36)$ \\
\hline & $\$ 112,263.68$ & $\$ 107,390.75$ & $\$ 55,450.01$ & $\$ 25,973.50$ & $\$ 25,973.50$ \\
\hline \multirow{4}{*}{$\begin{array}{r}\text { UW Expected Amount } \\
\text { UW Actual Expenses } \\
\text { Dver/Under Budget for } \\
\text { Quarter } \\
\text { W Total Under Budget }\end{array}$} & $\$ 25,908.50$ & $\$ 25,908.50$ & $\$ 25,908.50$ & $\$ 25,908.50$ & $\$ 103,634.00$ \\
\hline & $\$ 31,742.00$ & $\$ 31,075.00$ & $\$ 53,758.71$ & $\$ 40,437.07$ & $\$ 157,012.78$ \\
\hline & $(\$ 5,833.50)$ & $(\$ 5,166.50)$ & $(\$ 27,850.21)$ & $(\$ 14,528.57)$ & $(\$ 53,378.78)$ \\
\hline & $\$ 51,105.91$ & $\$ 45,939.41$ & $\$ 18,089.20$ & $\$ 3,560.63$ & $\$ 3,560.63$ \\
\hline \multirow{4}{*}{$\begin{array}{r}\text { NCSU Expected Amount } \\
\text { NCSU Actual Expenses } \\
\text { SU Over/Under Budget for } \\
\text { Quarter } \\
\text { NCSU Total Under Budget }\end{array}$} & $\$ 24,160.56$ & $\$ 24,160.56$ & $\$ 24,160.56$ & $\$ 24,160.56$ & $\$ 96,642.23$ \\
\hline & $\$ 18,613.24$ & $\$ 23,866.99$ & $\$ 48,251.08$ & $\$ 39,108.50$ & $\$ 129,839.81$ \\
\hline & $\$ 5,547.32$ & $\$ 293.57$ & $(\$ 24,090.52)$ & $(\$ 14,947.94)$ & $(\$ 33,197.58)$ \\
\hline & $\$ 61,157.77$ & $\$ 61,451.34$ & $\$ 37,360.81$ & $\$ 22,412.87$ & $\$ 22,412.87$ \\
\hline
\end{tabular}


FY 2013

\begin{tabular}{|c|c|c|c|c|c|}
\hline \multirow{3}{*}{$\begin{array}{l}\text { Total Approved } \\
\text { Actual Expenses }\end{array}$} & Q1 & Q2 & Q3 & Q4 ${ }^{*}$ & Y3 Totals \\
\hline & $\$ 49,942.00$ & $\$ 49,942.00$ & $\$ 49,942.00$ & $\$ 49,942.00$ & $\$ 199,768.02$ \\
\hline & $\$ 41,996.79$ & $\$ 77,133.01$ & $\$ 79,446.03$ & $\$ 27,165.69$ & $\$ 225,741.52$ \\
\hline \multirow{2}{*}{$\begin{array}{r}\text { Total Over/Under Budget for } \\
\text { Quarter } \\
\text { Total Under Budget }\end{array}$} & $\$ 7,945.21$ & $(\$ 27,191.01)$ & $(\$ 29,504.03)$ & $\$ 22,776.31$ & $(\$ 25,973.50)$ \\
\hline & $\$ 33,918.72$ & $\$$ \$6,727.71 & $(\$ 22,776.32)$ & $\$ 0.00$ & $\$ 0.00$ \\
\hline \multirow{2}{*}{$\begin{array}{l}\text { UW Expected Amount } \\
\text { UW Actual Expenses }\end{array}$} & $\$ 26,938.00$ & $\$ 26,938.00$ & $\$ 26,938.00$ & $\$ 26,938.00$ & $\$ 107,752.00$ \\
\hline & $\$ 17,402.09$ & $\$ 43,069.01$ & $\$ 38,558.33$ & $\$ 12,283.20$ & $\$ 111,312.63$ \\
\hline \multirow{2}{*}{$\begin{array}{r}\text { UW Over/Under Budget for } \\
\text { Quarter } \\
\text { UW Total Under Budget }\end{array}$} & $\$ 9,535.91$ & $(\$ 16,131.01)$ & $(\$ 11,620.33)$ & $\$ 14,654.80$ & $(\$ 3,560.63)$ \\
\hline & $\$ 13,096.54$ & $(\$ 3,034.47)$ & $(\$ 14,654.80)$ & $\$ 0.00$ & $\$ 0.00$ \\
\hline \multirow{4}{*}{$\begin{array}{r}\text { NCSU Expected Amount } \\
\text { NCSU Actual Expenses } \\
\text { U Over/Under Budget for } \\
\text { Quarter } \\
\text { TCSU Total Under Budget }\end{array}$} & $\$ 23,004.00$ & $\$ 23,004.00$ & $\$ 23,004.00$ & $\$ 23,004.00$ & $\$ 92,016.02$ \\
\hline & $\$ 24,594.70$ & $\$ 34,064.00$ & $\$ 40,887.70$ & $\$ 14,882.49$ & $\$ 114,428.89$ \\
\hline & $(\$ 1,590.70)$ & $(\$ 11,060.00)$ & $(\$ 17,883.70)$ & $\$ 8,121.51$ & $(\$ 22,412.87)$ \\
\hline & $\$ 20,822.18$ & $\$ 9,762.18$ & $(\$ 8,121.52)$ & $\$ 0.00$ & $\$ 0.00$ \\
\hline & & & \multicolumn{3}{|r|}{ Project Totals } \\
\hline & & & \multirow{3}{*}{\multicolumn{2}{|c|}{$\begin{array}{r}\text { Total Approved } \\
\text { Total Actual Expenses } \\
\text { Total Under Budget }\end{array}$}} & $\$ 616,073.25$ \\
\hline & & & & & $\$ 616,073.25$ \\
\hline & & & & & $\$ 0.00$ \\
\hline & & & \multirow{3}{*}{\multicolumn{2}{|c|}{$\begin{array}{l}\text { UW Expected Amount } \\
\text { UW Actual Expenses } \\
\text { UW Total Under Budget }\end{array}$}} & $\$ 330,963.16$ \\
\hline & & & & & $\$ 330,963.16$ \\
\hline & & & & & $\$ 0.00$ \\
\hline & & & \multirow{3}{*}{\multicolumn{2}{|c|}{$\begin{array}{l}\text { NCSU Expected Amount } \\
\text { NCSU Actual Expenses } \\
\text { NCSU Total Under Budget }\end{array}$}} & $\$ 285,110.09$ \\
\hline & & & & & $\$ 285,110.09$ \\
\hline & & & & & $\$ 0.00$ \\
\hline
\end{tabular}

\section{Current Quarter Budget}

UW spending for the current quarter was $\$ 12,283.20$. NCSU spending for the current quarter was $\$ 14,882.49$.

${ }^{*}$ The current quarter budget data (Q4) is expected amount spending by 9/30. The records are not yet all available.

Budget Explanations

All funds have been spent. 


\subsection{Progress Overview}

Task 1a - First-principles molecular dynamics (FPMD) calculations for pure (Li,K)Cl

Researchers: Amelia Bengtson (communication with Saumitra Saha, Hyo On Nam, Ridwan Sakidja)

Description: FPMD calculations were performed in VASP to determine the equation of state values (equilibrium volume, bulk modulus) for solid and molten $\mathrm{LiCl}$, $\mathrm{KCl},(\mathrm{Li}, \mathrm{K}) \mathrm{Cl}$

Status: We have calculated the equilibrium volumes, bulk modulus and selfdiffusion coefficients for 216-atom and 64 atom unit cells of $\mathrm{LiCl}, \mathrm{KCl}$ and the eutectic LiCl-KCl molten salts. Additional error analysis and convergence testing was performed to confirm FPMD results are well converged and the errors are as low as possible.

Issues/Concerns: none at this time

Corrective Actions: none at this time

Planned Completion 10/30/2011 (end of Year 1)

Date:

Percent Complete: $\quad 100 \%$

\section{Task 1b - First-principles molecular dynamics (FPMD) calculations for Sr solute}

Researchers: Hyo On Nam (communication with Katharina Vörtler)

Description: Prediction of properties with the Sr solute from the first-principles molecular dynamics calculations

Status: FPMD calculations were conducted to study the solute $\mathrm{Sr}$ behavior in the $\mathrm{LiCl}-\mathrm{KCl}$ at $773 \mathrm{~K}\left(500^{\circ} \mathrm{C}\right)$. Diffusion coefficient and redox potential of $\mathrm{Sr}$ were estimated and structural analysis were performed. Simulations with other solutes $(\mathrm{Zr}(\mathrm{IV}) / \mathrm{Zr}(0), \quad \mathrm{Eu}(\mathrm{III}) / \mathrm{Eu}(\mathrm{II}), \quad \mathrm{Eu}(\mathrm{II}) / \mathrm{Eu}(0)$, $\mathrm{Nd}(\mathrm{III}) / \mathrm{Nd}(0)$, and $\mathrm{Ce}(\mathrm{III}) / \mathrm{Ce}(0))$ in the $\mathrm{LiCl}-\mathrm{KCl}$ are also analyzed for comparison.

Issues/Concerns: none at this time

Corrective Actions: none at this time

Planned Completion 4/1/2012 (end of Q2, Year 2)

Date:

Percent Complete: $\quad$ 100\% 


\section{Task 1c - First-principles molecular dynamics (FPMD) calculations for La solute}

Researchers: Hyo On Nam (communication with Katharina Vörtler)

Description: Prediction of properties with the La solute from the first-principles molecular dynamics calculations

Status: FPMD calculations were conducted to study the solute La behavior in the $\mathrm{LiCl}-\mathrm{KCl}$ at $773 \mathrm{~K}\left(500^{\circ} \mathrm{C}\right)$. Diffusion coefficient and redox potential of La were estimated and structural analysis were performed. Simulations with other solutes $(\mathrm{Zr}(\mathrm{IV}) / \mathrm{Zr}(0), \quad \mathrm{Eu}(\mathrm{III}) / \mathrm{Eu}(\mathrm{II}), \quad \mathrm{Eu}(\mathrm{II}) / \mathrm{Eu}(0)$, $\mathrm{Nd}(\mathrm{III}) / \mathrm{Nd}(0)$, and $\mathrm{Ce}(\mathrm{III}) / \mathrm{Ce}(0))$ in the $\mathrm{LiCl}-\mathrm{KCl}$ are also analyzed for comparison

Issues/Concerns: none at this time

Corrective Actions: none at this time

Planned Completion 10/01/2012 (end of Year 2)

Date:

Percent Complete: $\quad 100 \%$

\section{Task 1d - First-principles molecular dynamics (FPMD) calculations for U solute}

Researchers: Hyo On Nam (communication with Katharina Vörtler)

Description: Prediction of properties with the U solute from the first-principles molecular dynamics calculations

Status: FPMD calculations were conducted to study the solute $\mathrm{U}$ behavior in the $\mathrm{LiCl}-\mathrm{KCl}$ at $773 \mathrm{~K}\left(500^{\circ} \mathrm{C}\right)$. Diffusion coefficient and redox potential of $\mathrm{U}$ were estimated and structural analysis were performed. Simulations with other solutes $(\mathrm{Zr}(\mathrm{IV}) / \mathrm{Zr}(0), \quad \mathrm{Eu}(\mathrm{III}) / \mathrm{Eu}(\mathrm{II}), \quad \mathrm{Eu}(\mathrm{II}) / \mathrm{Eu}(0)$, $\mathrm{Nd}(\mathrm{III}) / \mathrm{Nd}(0)$, and $\mathrm{Ce}(\mathrm{III}) / \mathrm{Ce}(0))$ in the $\mathrm{LiCl}-\mathrm{KCl}$ are also analyzed for comparison

Issues/Concerns: none at this time

Corrective Actions: none at this time

Planned Completion 4/1/2013 (end of Q2, Year 3)

Date:

Percent Complete: $\quad 100 \%$ 


\section{Task 2a-Interatomic potential development for $\mathrm{Sr}$}

Researchers: Ridwan Sakidja, Hyo On Nam

Description: Develop basic potential for Sr that can be used in interatomic-potential (IPMD) methods.

Status: A Sr model pair potential based on ab-initio calculations has been developed using force-matching algorithm. Developed $\mathrm{Sr}$ potential is validated by comparing the RDFs and self-diffusion coefficients.

Issues/Concerns: none at this time

Corrective Actions: none at this time

Planned Completion 4/1/2012 (end of Q2, Year 2)

Date:

Percent Complete: $\quad 100 \%$

\section{Task 2b-Interatomic potential development for La}

Researchers: Ridwan Sakidja

Description: Develop basic potential for La that can be used in interatomic-potential (IPMD) methods.

Status: Not pursued.

Issues/Concerns: We have discovered that this potential was already developed in the literature (see the reference, J. Phys. Chem. B 112 (2008) 1177-1183 [1])

Corrective Actions: We are no longer planning to develop this potential as it has already been developed in the literature.

Planned Completion 10/1/2012 (end of Year 2)

Date:

Percent Complete: $\quad 100 \%$ 


\title{
Task 2c-Interatomic potential development for $\mathbf{U}$
}

Researchers: Ridwan Sakidja

Description: Develop basic potential for U that can be used in interatomic-potential (IPMD) methods.

Status: Not pursued.

Issues/Concerns: We have discovered that this potential was already developed in the literature (see the reference, J. Phys. Chem. B 112 (2008) 1177-1183 [1])

Corrective Actions: We are no longer planning to develop this potential as it has already been developed in the literature.

Planned Completion 4/1/2013 (end of Q2, Year 3)

Date:

Percent Complete: $\quad 100 \%$

\section{Task 3a - Interatomic potential molecular dynamics (IPMD) calculations for pure (Li,K)Cl (validate FPMD) - Part 1}

Researchers: Amelia Bengtson

Description: The properties of particular interest are equation of state values (equilibrium volume, bulk modulus) for solid and molten LiC-KCl. IPMD calculations are significantly faster than FPMD calculations, and thus are an efficient way to refine input parameter choices, convergence and equilibration of the molecular dynamics calculations.

Status: We have calculated the equilibrium volumes, bulk modulus and selfdiffusion coefficients for $\mathrm{LiCl}, \mathrm{KCl}$ and the eutectic $\mathrm{LiCl}-\mathrm{KCl}$ molten salts using FPMD and IPMD. IPMD calculations were critical in proving FPMD results are converged with respect to unit cell size and simulation time.

\author{
Issues/Concerns: none at this time \\ Corrective Actions: none at this time \\ Planned Completion 10/30/2011 (end of Year 1) \\ Date:
}

Percent Complete: $\quad 100 \%$ 


\section{Task 3a - Interatomic potential molecular dynamics (IPMD) calculations for pure (Li,K)Cl (validate FPMD) - Part 2}

Researchers: Brahmananda Chakraborty and Jin Wang

Description: Diffusion matrix for multicomponent diffusion

Status: Completed

Issues/Concerns: none at this time

Corrective Actions: none at this time

Planned Completion 10/30/2011 (end of Year 1)

Date:

Percent Complete: $\quad 100 \%$

\section{Task 3b - Free Energy Simulations development/analysis}

Researchers: Jin Wang and Brahmananda Chakraborty

Description: Develop mixing free energies to calculate solute activity.

Status: The 2PT method, as described in the proposal, is employed to calculate the free energy for the molten salt system.

Issues/Concerns: none at this time

Corrective Actions: none at this time

Planned Completion 4/1/2012 (end of Q2, Year 2)

Date:

Percent Complete: $\quad 100 \%$

\section{Task 3c - Prediction of properties with the Sr solute}

Researchers: Jin Wang and Brahmananda Chakraborty

Description: Prediction of properties with the Sr solute from the thermodynamics and free energy calculations.

Status: The 2PT method was used to predict the molar free energy, entropy and specific heat of Sr(II) ion in the eutectic LiCl-KCl mixture. Born-HugginsMeyer (BHM) interatomic potential for Sr(II) in the LiCl-KCl developed at UW-Madison was used.

Issues/Concerns: none at this time

Corrective Actions: none at this time

Planned Completion 10/1/2012 (end of Year 2)

Date:

Percent Complete: $\quad$ 100\% 


\section{Task 3d - Prediction of properties with the La solute}

Researchers: Jin Wang and Brahmananda Chakraborty

Description: Prediction of properties with the La solute from the thermodynamics and free energy calculations

Status: The 2PT method was used to predict the molar free energy, entropy and specific heat of $\mathrm{La}(\mathrm{III})$ ion in the eutectic LiCl-KCl mixture. The interatomic potential for $\mathrm{La}(\mathrm{III})$ in the $\mathrm{LiCl}-\mathrm{KCl}$ developed by Salanne et al. (J. Phys. Chem. B 112 (2008) 1177-1183 [1]) was used.

Issues/Concerns: none at this time

Corrective Actions: none at this time

Planned Completion 4/1/2013 (end of Q2, Year 3)

Date:

Percent Complete: $\quad 100 \%$

\section{Task 3e - Prediction of properties with the U solute}

Researchers: Jin Wang and Brahmananda Chakraborty

Description: Prediction of properties with the U solute from the thermodynamics and free energy calculations.

Status: The 2PT method was used to predict the molar free energy, entropy and specific heat of $\mathrm{U}(\mathrm{III})$ ion in the eutectic $\mathrm{LiCl}-\mathrm{KCl}$ mixture. The interatomic potential for U(III) in the LiCl-KCl developed by Salanne et al. (J. Phys. Chem. B 112 (2008) 1177-1183 [1]) was used.

Issues/Concerns: none at this time

Corrective Actions: none at this time

Planned Completion 10/1/2013 (end of Q4, Year 3)

Date:

Percent Complete: $\quad 100 \%$ 


\subsection{Presentations and publications}

Peer-reviewed Publications:

- One journal article is published, one accepted, and two submitted. Two ANS transactions have been published.

o Brahmananda Chakraborty, Jin Wang and Jacob Eapen, Multicomponent diffusion in Molten LiCl-KCl: Dynamical correlations and divergent Maxwell-Stefan diffusivities, Physical Review E 87, 052312 (2013).

o Jin Wang, Brahmananda Chakraborty and Jacob Eapen, Free energy of molten salts with the 2PT method, accepted for publication, Physical Chemistry Chemical Physics (2013).

o A. Bengtson, H. Nam, D. Morgan et al., First-principles molecular dynamics modeling of the LiCl-KCl molten salt system, submitted, Computational Materials Science.

o H. Nam, A. Bengtson, D. Morgan, Redox condition in molten salt and solute behavior: A first-principles molecular dynamics study, submitted, Journal of Nuclear Materials.

o Brahmananda Chakraborty, Jin Wang and Jacob Eapen, Multicomponent diffusion in Molten LiCl-KCl Mixture, ANS Transactions, 106, 1284 (2012).

o Jin Wang and Jacob Eapen, Free energy of molten salt (KCl) for reprocessing applications, ANS Transactions, 108, 451 (2013).

Talks:

- Invited international talk at NUMAT in Japan.

o A. Bengtson, H.O. Nam, R. Sakidja, S. Saha, D. Morgan, First Principles Molecular Dynamics Modeling of Solute Thermokinetics in Molten Salts for Nuclear Energy Applications, NuMat 2012: Nuclear Materials conference, 21-25 October 2012, Osaka, Japan 


\title{
3. Technical Report from North Carolina State University (Task 3)
}

\author{
Researchers: Jin Wang, Brahmananda Chakraborty and Jacob Eapen
}

This section reports on results from Task 3

\subsection{Introduction}

The separation of actinides and fission products is a central step in closing the nuclear fuel cycle. The advantage of having a closed fuel cycle includes a reduction of high-level nuclear waste and the optimal utilization of the fissionable isotopes. While aqueous-based (wet) processes are wellestablished there is a current interest for high temperature pyrometallurgical processes [2-4] that utilize molten salts. In the pyrometallurgical recycling process, the spent nuclear fuel is dissolved in a molten salt followed by the extraction of actinides. Originally developed for spent metallic fuel from the fast reactors, pyro processes can be also used for spent fuel from the current light water reactors (LWRs) using electroreduction, electrorefining, and pyropartitioning; they are also regarded to be more proliferation-resistant than the traditional wet processes. Molten mixtures of $\mathrm{LiCl}$ and $\mathrm{KCl}$ are strong candidates for pyrometallurgical recycling process [2-7] as well as in other industrial chemical processes [8]. In particular, an eutectic mixture of $\mathrm{LiCl}-\mathrm{KCl}$ is attractive as a solvent because of its lower melting point (626K) relative to that of the constituent salts (883K for $\mathrm{LiCl}$ and $1043 \mathrm{~K}$ for $\mathrm{KCl}$ ).

Absolute thermodynamic properties [9-15] are needed for optimizing the back-end of the nuclear fuel cycle, which entails the crucial step of separation of actinides from the spent nuclear fuel, followed by the development of new fuel through recycling. The term 'absolute' is used in the sense that the thermodynamic properties can be directly evaluated through the partition function for a given state in the canonical ensemble; there is however, an arbitrary reference energy which is associated with the potential energy of the system.

Reprocessing of nuclear fuel involves a large number of fissionable actinides [2, 7] as well as nonfissionable fission products; the separation processes typically depend on the chemical activity of the species, which in turn depends on the relative free energy [4]. Thermodynamic integration (TI), which is the method of choice for a variety of applications [11, 14, 16-19], especially with molecular dynamics (MD) simulations, may not be the most optimal choice for nuclear reprocessing applications given the inordinate number of species that are targeted for separation. In several instances, TI can be limited by the optimal choice of the integration path that is not known a priori, and is also constrained in having a reverse path that implicitly excludes a first order phase change. Another challenge arises in proscribing appropriate reference systems, which can allow computation of absolute thermodynamic properties. While TI can successfully predict free energy differentials with actinide transmutation [4], integration paths typically require trajectories that span over $1 \mathrm{~ns}$, which is expensive by current standards. A problem of a different nature may arise with actinide dissolution in molten salts - depending on the volume fraction, the interaction strength and the thermodynamic state, the dissolved species may form glassy states or even undergo a first-order liquid-liquid phase transition [20-24], which is somewhat poorly understood currently. Very strong attractive interactions have shown to result in amorphous transition in a model nano-colloidal system with a volume fraction as low as 5\% [25]. Thus it would be profitable to identify a 
methodology that can obviate some or all of the aforesaid possible drawbacks for systems with large number of species in complex liquid forms.

Originally developed by Lin, Blanco and Goddard [26], the two-phase thermodynamic (2PT) model has recently gained wide attention and success in predicting absolute thermodynamic properties for several types of fluids $[9,10,12,13,15,27-30]$. Motivated by the approach of evaluating the partition function in the solid state using idealized harmonic oscillators, the central idea behind the 2PT methodology is to construct the partition function of a complex liquid state through a superposition of solid-like and gas-like vibrational density of states (DoS). An exact representation of the idealized states can be derived by treating the gas-like state through hard-sphere interactions with weighting functions provided by the Carnahan-Starling equation of state [31], and assuming the solid-like state to be a collection of independent harmonic oscillators. The thermodynamic properties of a liquid system thus can be shown to be a linear combination of the properties of a set of harmonic oscillators (solid-like) and a set of hard spheres (gas-like).

In this investigation, we show that the absolute thermodynamic properties of $\mathrm{LiCl} / \mathrm{KCl}$ molten salts with and without actinide solutes can be accurately determined from the 2PT method. The 2PT free energy is in excellent agreement with that determined from the thermodynamic integration (TI) method, with relative errors of $1 \%$ or less, typically. The melting point of ionic $\mathrm{KCl}(1025 \mathrm{~K})$ compares favorably with the experimental data $(1041 \mathrm{~K})$; the entropy of the melt phase also show good conformity with the recommended data from NIST. We then determine the absolute free energy and entropy which are currently unknown for a eutectic mixture of $\mathrm{LiCl}$ and $\mathrm{KCl}$ for temperatures ranging from $773 \mathrm{~K}$ to $1300 \mathrm{~K}$. From the partitioning of the DoS, which is uniquely possible in the 2PT method, we show that the $\mathrm{Li}^{+}$ions in the LiCL-KCl mixture are dominantly solid-like even at temperatures as high as $1300 \mathrm{~K}$. For chemical and nuclear applications the solidlike states can potentially impose practical limits, for example, to the amount of dissolved species that can be practically accommodated during reprocessing. Finally, we evaluate the free energies of dissolved $\mathrm{U}, \mathrm{La}$ and $\mathrm{Sr}$ in eutectic $\mathrm{LiCl}-\mathrm{KCl}$ mixtures. 


\subsection{IPMD Calculations for Pure LiCl-KCl Mixtures}

The LiCl-KCl mixture has been investigated in the past both experimentally and computationally, however, the multicomponent diffusional properties which are critical to the nuclear and chemical applications are currently not known in great detail. Collective effects in multicomponent systems give rise to interesting behavior such as the Chemla effect in melts and solutions - the faster mobility of the larger ions relative to the smaller ions above a critical concentration of the larger ions [32-36], and vanishingly small charge transport which can arise from strong anion-cation binding [37, 38]. Though the principles of multicomponent diffusion are well-established [39, 40], experimental data and analyses of ternary and higher order systems - see [41, 42] for recent work, in general, are limited in comparison to simpler binary systems [43].

At a theoretical level, the Maxwell-Stefan (MS) framework [44, 45] may offer several advantages such as fewer number of diffusion coefficients, empirical correlation to self-diffusivities that aid in model development, relatively smaller dependence on concentrations, and in most cases, a symmetric, quasi-positive, diffusion matrix [46]. In the recent past, MS framework has been applied in several theoretical, computational and experimental analyses [47-55]. Since experiments can only measure the Fickian diffusivities, MS diffusivities can be transformed to Fickian using the thermodynamic matrix that arises from the non-ideality of the multi-component system [45, 56, 57]. Following Kirkwood and Buff [58], the thermodynamic matrix is expressible in terms of integrals of the (partial) radial distribution functions of the species. Fickian experimental data can also be transformed to MS diffusivities, however, owing to the smaller number of MS diffusivities for ternary and higher-order systems, only the transformation from MS to Fickian is well-posed while the reverse transformation is over-determined [50].

In the MS framework the driving force is regarded to be counterbalanced by molecular friction which is proportional to the relative velocity between the species. We adopt a mole-fraction $(x)$ based formalism and evaluate the MS diffusivities of $\mathrm{LiCl}-\mathrm{KCl}$ mixture at different temperatures and compositions from the Onsager dynamical matrix $(\boldsymbol{\Lambda})$ that is evaluated from molecular dynamics (MD) simulations using the Green-Kubo time-correlation approach. The dynamical correlations show that the $\mathrm{Li}-\mathrm{Li}$ and $\mathrm{Li}-\mathrm{Cl}$ ion pairs show a conspicuous cage effect or backscattering at $773 \mathrm{~K}$ which surprisingly remains strong with increasing temperature. In contrast, both $\mathrm{K}-\mathrm{K}$ and $\mathrm{Cl}-\mathrm{Cl}$ correlations show less pronounced backscattering which diminishes as temperature is increased. Finally from the Onsager dynamical matrix we evaluate the MS diffusivities for different temperatures $(773 \mathrm{~K}-1200 \mathrm{~K}, 1 \mathrm{bar})$ and compositions $\left(x_{\mathrm{KCl}}=0.42-0.63\right)$. For the positive $\mathrm{K}-\mathrm{Li}$ ion pair we observe a theoretically consistent but unusual change of sign and divergent-like behavior for the MS diffusivity even though Onsager coefficients change relatively smoothly across the different temperatures and compositions. Negative MS diffusivities have been experimentally observed in the past for electrolytic solutions; the current results portray an analogous behavior where MS diffusivity of the like-like ion tends to diverge at a critical composition (at the same temperature and pressure), and at a critical temperature (at the same composition and pressure). The diffusivities of the like-unlike ions in contrast show only minor variation across the critical temperature and composition which is consistent with the experimental data on compositions for ionic systems. We further verify the thermodynamic consistency by showing that the MS diffusivities for the $\mathrm{K}-\mathrm{Li}$ ion pair correspond to a non-negative entropy production at all temperatures and compositions. We conclude by discussing the possible implications of the observed behavior from the simulations. 


\subsubsection{Onsager Coefficients}

The linear phenomenological theory postulates that the diffusive fluxes in an $n$-component system are linear homogeneous functions of thermodynamic forces as given by [39, 40]

$\hat{\mathbf{J}} \equiv \boldsymbol{\Lambda \mathbf { X }}$

where $\hat{\mathbf{J}}$ and $\hat{\mathbf{X}}$ are the diffusive flux and thermodynamic force vectors, respectively, and $\boldsymbol{\Lambda}$ is the Onsager matrix containing the phenomenological coefficients. For describing the diffusion fluxes, we have adopted a mole based framework where the molar flux is defined relative to a reference velocity $\hat{\mathbf{V}}^{\bullet}$ as

$$
\hat{\mathbf{J}}_{i}^{\bullet}=c_{i}\left(\hat{\mathbf{U}}_{i}-\hat{\mathbf{V}}^{\bullet}\right)=c x_{i}\left(\hat{\mathbf{U}}_{i}-\hat{\mathbf{V}}^{\bullet}\right) \quad(i=1,2, \ldots, n)
$$

where $x_{i}, c_{i}$ and $\hat{\mathbf{U}}_{i}$ are the mole fraction, molar density and velocity, respectively, of the $i^{\text {th }}$ species. Two different reference frames are followed in this analysis; the first one is used for defining the macroscopic (molar) flux which is defined relative to the mean molar velocity as given in Eqn. (3.2.3) in the formulation of MS diffusion theory.

$$
\hat{\mathbf{V}}^{\bullet}=\sum_{i=1}^{n} x_{i} \hat{\mathbf{U}}_{i}
$$

The second frame is for describing the microscopic flux used in the molecular dynamics (MD) simulations for evaluating the Onsager coefficients. For equilibrium MD simulations the most natural frame is barycentric as momentum conservation leads to a constant center-of-mass velocity which is then set to zero to prevent a rigid body translation of the simulation cell. The Onsager coefficients $\left(\Lambda_{i k}\right)$ are evaluated from the linear response theory using equilibrium MD simulations [59]. First the microscopic diffusion (molar) flux of $k^{\text {th }}$ species relative to the reference velocity $\mathbf{V}^{\mathbf{0}}$ is defined as

$$
\mathbf{j}_{k}^{0}(\mathbf{r}, t)=\sum_{l=1}^{N_{k}}\left[\mathbf{u}_{k}^{l}(t)-\mathbf{V}^{0}(t)\right] \delta\left(\mathbf{r}(t)-\mathbf{r}_{l}(t)\right)
$$

where $l$ denote the atom/ion index, and $N_{k}$ is the total number of atoms/ions for the $k^{\text {th }}$ species. The superscript 0 signifies the barycentric reference frame against which the microscopic molar flux is defined, and the delta function serves to localize the atoms within an arbitrarily small region in space $(\Omega)$. Integrating over all $\Omega$, the volume-averaged, microscopic flux can be expressed as

$$
\mathfrak{I}_{k}^{0}(t)=\int_{\Omega} \mathbf{j}_{k}(\mathbf{r}, t)=\sum_{l=1}^{N_{k}}\left[\mathbf{u}_{k}^{l}(t)-\mathbf{V}^{0}(t)\right]
$$

The volume averaged diffusive flux thus is simply the sum of the atom velocities relative to the barycentric velocity which is constrained to be zero. From the linear response theory [40], the Onsager coefficients can be evaluated as time correlations of flux fluctuations [59]. The elements of the Onsager dynamical matrix can be shown to be 


$$
\Lambda_{i k}^{0}=\frac{1}{3 N} \int_{0}^{\infty} d t\left\langle\Im_{i}^{0}(t) \Im_{k}^{0}(0)\right\rangle \equiv \frac{1}{3 N} \int_{0}^{\infty} d t \Psi_{i k}^{0}(t)
$$

where $N$ is the total number of atoms/ions in the system, and $\Psi(t)$ denotes the ensemble average of the diffusive molar flux correlation. The Onsager matrix elements can be also determined from the atomic displacements as

$$
\Lambda_{i k}^{0}=\frac{1}{6 N} \lim _{t \rightarrow \infty} \frac{d}{d t}\left\langle\left[\mathbf{r}_{i}^{0}(t)-\mathbf{r}_{i}^{0}(0)\right] \cdot\left[\mathbf{r}_{k}^{0}(t)-\mathbf{r}_{k}^{0}(0)\right]\right\rangle
$$

The expression in Eqn. (3.2.7) which is known as the Einstein form is formally equivalent to that given in Eqn. (3.2.6) which is known as the Green-Kubo form. Even though only $(n-1) \times(n-1)$ Onsager phenomenological coefficients are independent, we determine all the $n \times n$ Onsager coefficients as they can be useful for diffusion model development [56]. In the barycentric frame, the following relationships exist among the Onsager coefficients

$$
\sum_{i=1}^{n} M_{i} \Lambda_{i k}^{0}=0
$$

and $\Lambda_{i k}^{0}=\Lambda_{k i}^{0}$

where $M$ denotes the molar mass. Eqn. (3.2.8) can be derived from the conservation of mass while Eqn. (3.2.9) is justified from the microscopic reversibility, or equivalently, from the principle of detailed balance, first espoused by Onsager [60, 61].

The thermodynamic force is usually expressed in terms of the gradient of the chemical potential ( $\boldsymbol{\mu})$. The Gibbs-Duhem equation given by [40]

$$
\sum_{i=1}^{n} x_{i}\left(\nabla \mu_{i}\right)_{T, p}=0
$$

provides a functional relationship between the species chemical potentials. Eqns. (3.2.8) and (3.2.10) are used to reduce the dimensionality of the Onsager matrix and the set of MS diffusion equations.

\subsubsection{Maxwell-Stefan (MS) Diffusion}

First proposed by Maxwell and extended by Stefan, the MS approach assumes a driving force ( $\hat{\mathbf{x}}$ ) which is proportional to the relative velocity between the species in the system [44]. We have adopted the framework developed by Krishna and Wesselingh [45] where the thermodynamic force is expressed as 
$-\frac{\nabla \mu_{i}}{R T}=\sum_{\substack{k=1 \\ k \neq i}}^{n} \frac{x_{k}}{\bigoplus_{i k}}\left(\hat{\mathbf{U}}_{i}-\hat{\mathbf{U}}_{k}\right)$

where $\bigoplus_{i k}$ is defined to be the MS diffusivity (having the common units of $\mathrm{L}^{2} / \mathrm{T}$ ). The above equation can be expressed in terms of molar fluxes relative to the mean molar velocity as [45]

$-\frac{x_{i}}{R T} \nabla \mu_{i}=\sum_{\substack{k=1 \\ k \neq i}}^{n} \frac{1}{c \bigoplus_{i k}}\left(x_{k} \mathbf{J}_{i}^{\bullet}-x_{i} \mathbf{J}_{k}^{\bullet}\right)$

and recast into a matrix form as

$\hat{\mathbf{X}}^{\bullet}=\mathbf{B} \hat{\mathbf{J}}^{\bullet}$

where $\hat{\mathbf{X}}^{\bullet}$ and $\hat{\mathbf{J}}^{\bullet}$ are vectors of dimension (n-1), and $\mathbf{B}$ is a (n-1) by $(n-1)$ matrix, the elements of which are given by [55]

$B_{i j}=\delta_{i j}\left(\frac{x_{i}}{\bigoplus_{i n}}+\sum_{\substack{k=1 \\ k \neq i}}^{n} \frac{x_{k}}{\bigoplus_{i k}}\right)+\left(1-\delta_{i j}\right) x_{i}\left(\frac{1}{\bigoplus_{i n}}-\frac{1}{\bigoplus_{i j}}\right)$

where $\delta_{i j}$ is the Kronecker delta function. Eqn. (3.2.13) can be now inverted to give

$\hat{\mathbf{J}}^{\bullet}=\mathbf{B}^{-1} \hat{\mathbf{X}}^{\bullet} \equiv \hat{\Lambda}^{\bullet} \hat{\mathbf{X}}^{\bullet}$

From MD simulations the Onsager matrix elements are evaluated in a barycentric frame while the above equation needs the Onsager matrix elements in the mean molar velocity frame. After the appropriate transformation and dimension reduction from $n$ to $(n-1), \mathbf{K}$ ( $\equiv \mathbf{B}^{-1}$ ) matrix elements for a ternary system can be expressed in terms of Onsager coefficients as [55]

$$
\begin{aligned}
& K_{11}=\left(1-x_{1}\right)\left(\frac{\Lambda_{11}}{x_{1}}-\frac{\Lambda_{13}}{x_{3}}\right)-x_{1}\left(\frac{\Lambda_{21}}{x_{1}}-\frac{\Lambda_{23}}{x_{3}}+\frac{\Lambda_{31}}{x_{1}}-\frac{\Lambda_{33}}{x_{3}}\right) \\
& K_{12}=\left(1-x_{1}\right)\left(\frac{\Lambda_{12}}{x_{2}}-\frac{\Lambda_{13}}{x_{3}}\right)-x_{1}\left(\frac{\Lambda_{22}}{x_{2}}-\frac{\Lambda_{23}}{x_{3}}+\frac{\Lambda_{32}}{x_{2}}-\frac{\Lambda_{33}}{x_{3}}\right) \\
& K_{21}=\left(1-x_{2}\right)\left(\frac{\Lambda_{21}}{x_{1}}-\frac{\Lambda_{23}}{x_{3}}\right)-x_{2}\left(\frac{\Lambda_{11}}{x_{1}}-\frac{\Lambda_{13}}{x_{3}}+\frac{\Lambda_{31}}{x_{1}}-\frac{\Lambda_{33}}{x_{3}}\right) \\
& K_{22}=\left(1-x_{2}\right)\left(\frac{\Lambda_{22}}{x_{2}}-\frac{\Lambda_{23}}{x_{3}}\right)-x_{2}\left(\frac{\Lambda_{12}}{x_{2}}-\frac{\Lambda_{13}}{x_{3}}+\frac{\Lambda_{32}}{x_{2}}-\frac{\Lambda_{33}}{x_{3}}\right)
\end{aligned}
$$

From the definition of MS diffusivities, the elements of the $\mathbf{B}$ matrix can be written as 


$$
\mathbf{B}=\left[\begin{array}{ll}
\frac{x_{1}}{Ð_{13}}+\frac{x_{2}}{Ð_{12}}+\frac{x_{3}}{Ð_{13}} & x_{1}\left(\frac{1}{Ð_{13}}-\frac{1}{Ð_{12}}\right) \\
x_{2}\left(\frac{1}{Ð_{23}}-\frac{1}{Ð_{12}}\right) & \frac{x_{1}}{Ð_{21}}+\frac{x_{2}}{Ð_{23}}+\frac{x_{3}}{Ð_{23}}
\end{array}\right]
$$

And finally, the MS diffusivities for ternary systems can be expressed as

$$
\begin{aligned}
& \bigoplus_{12}=\left[B_{11}-\left(1+\frac{x_{3}}{x_{1}}\right) B_{12}\right]^{-1} \\
& \bigoplus_{23}=\left[B_{22}+\left(\frac{x_{1}}{x_{2}}\right) B_{21}\right]^{-1} \\
& \bigoplus_{13}=\left[B_{11}+\left(\frac{x_{2}}{x_{1}}\right) B_{12}\right]^{-1}
\end{aligned}
$$

Using MD simulations we have first computed the Onsager $\boldsymbol{\Lambda}$ matrix elements followed by the evaluation of the matrix elements of $\mathbf{B}$ from $\mathbf{K}^{-1}$ which are then used to compute the MS diffusivities using Eqn. (3.2.18). The MS diffusivities are symmetric and they exist only for different species. They can however, be state dependent, and as shown later they can also tend to diverge and become negative for $\mathrm{LiCl}-\mathrm{KCl}$ mixtures at certain critical thermodynamic states.

The entropy production is used to verify whether the observed diffusivities are consistent with the II law of thermodynamics. The entropy production rate per unit volume is given by [45]

$$
\dot{\sigma}=c R \sum_{\substack{i=1 \\ i>k}}^{n} \frac{x_{i} x_{k}}{\bigoplus_{i k}}\left(\hat{\mathbf{U}}_{i}-\hat{\mathbf{U}}_{k}\right)^{2}
$$

where $R$ is the universal gas constant. Based on the above the following inequality holds for all thermodynamic states [62]

$$
\begin{array}{r}
\beta_{a b}=\sum_{\substack{i=1 \\
i \neq a}}^{n}\left(\frac{x_{i} x_{a}}{\bigoplus_{i a}}\right) \sum_{\substack{j=1 \\
j \neq b}}^{n}\left(\frac{x_{j} x_{b}}{\bigoplus_{j b}}\right)-\left(\frac{x_{a} x_{b}}{\bigoplus_{a b}}\right)^{2} \geq 0 \\
a, b=(1, \ldots, n), a \neq b
\end{array}
$$

Thus the number of constraints is equal to the number of MS diffusion coefficients; for a ternary system there are just three constraints. We will show later that the entropy remains positive even for state points where negative MS diffusivities are observed, and in the limiting case of divergent $(+\infty$, $-\infty) \mathrm{MS}$ diffusivities for the $\mathrm{K}-\mathrm{Li}$ ion pair. 


\subsubsection{Molecular Dynamics (MD/IPMD) Simulations}

Dynamical correlations and Onsager coefficients are computed using custom modules incorporated in DL-POLY [63], a classical molecular dynamics program. The molecular system consists of 4096 ions interacting through a long-ranged electrostatic potential and a Born-Mayer-Huggins (BHM) short-ranged potential. Originally developed by Fumi and Tosi [64], the rigid-ion potential is able to predict several thermodynamic, structural and transport properties of molten $\mathrm{LiCl}-\mathrm{KCl}$ mixtures $[35,65]$ and other alkali halides [64] with reasonable accuracy. Linear response theory is generally found to be tractable for evaluating many-body diffusional and thermal transport properties [35, 65]. The effect of polarization however, is important for multicomponent systems as illustrated in [6669] although it tends to diminish for monovalent systems such as $\mathrm{LiCl}-\mathrm{KCl}$. The functional form of short-ranged part of the BHM potential is given by

$$
\Phi_{i j}^{s h o r t}=B_{i j} e^{-\alpha r_{i j}}-\frac{C_{i j}}{r_{i j}^{6}}-\frac{D_{i j}}{r_{i j}^{8}}
$$

where $r_{i j}$ is the interionic separation between two ions, $i$ and $j$, and $\alpha, B, C$ and $D$ are constants [35, 65]. While the first term corresponds to the electron cloud repulsion, the second and third terms account for the dipole-dipole and the dipole-quadrupole dispersion interactions, respectively.

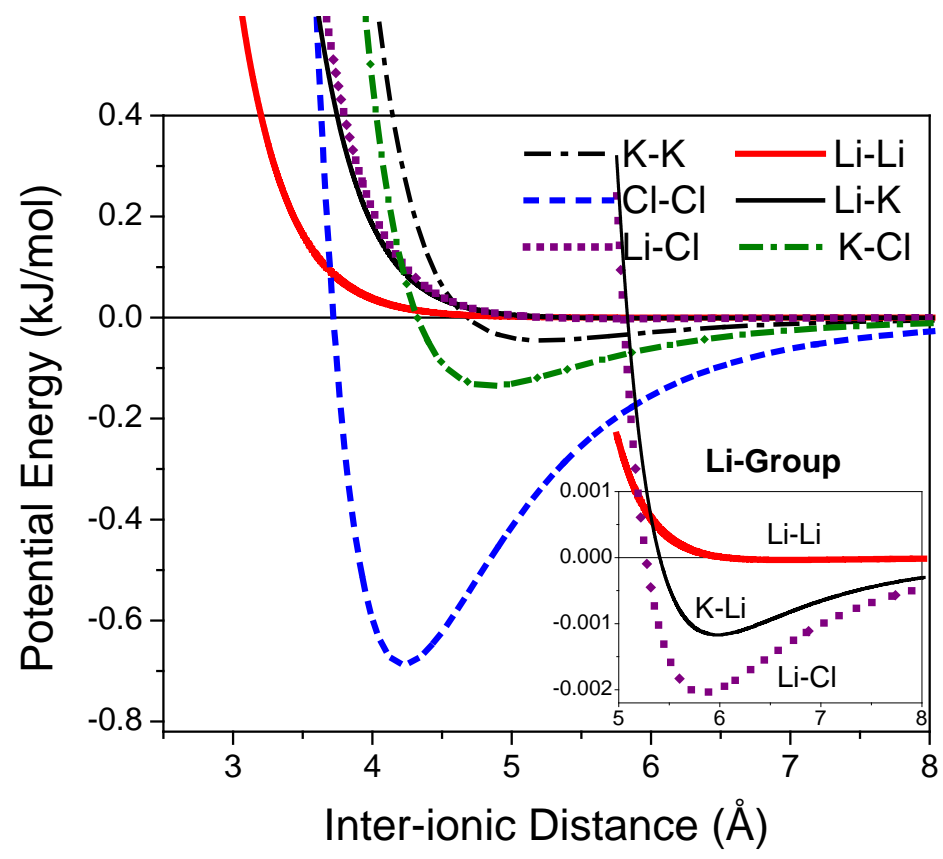

Figure 3.1. Short-ranged BHM interactions for $\mathrm{LiCl}-\mathrm{KCl}$ mixture. (Inset) The interactions for the $\mathrm{Li}$ group ( $\mathrm{Li} \mathrm{Li}, \mathrm{Li}-\mathrm{K}$ and $\mathrm{Li}-\mathrm{Cl}$ ) on a magnified scale.

As seen from Figure 3.1, the strongest short-ranged interaction (with the deepest attractive well) is between the $\mathrm{Cl}$ ions while the weakest is between the $\mathrm{Li}$ ions. In general, the interactions among the $\mathrm{Li}$ group of atoms (see inset) is significantly weaker than those for $\mathrm{K}$ and $\mathrm{Cl}$ ions. 
MD simulations are performed with 4096 ions in a cubic cell with periodic boundary conditions (PBC). The system is first equilibrated in an NPT ensemble (Nosé-Hoover) for 600-700 ps followed by another $\sim 300 \mathrm{ps}$, typically, for collecting the dynamical correlations in the NVE ensemble. Both the velocity correlations and the Einstein form are computed which are averaged over 3000 independent (overlapped) sets for each state point [70]. The Onsager coefficients are further averaged over 8-12 independent runs (with a total of $\sim 6$ ns for each state point) to improve the statistics of the correlations. The equations of motion are numerically integrated by the Velocity Verlet algorithm using a time step of $1 \mathrm{fs}$. The electrostatic interactions are modeled by Ewald sum with a cut-off radius of $24 \AA$. The simulations are performed at different temperatures and compositions at a constant pressure of 1 bar.

\subsubsection{Dynamic Correlations}

We will now discuss the temporal behavior of the diffusive flux correlations, the integral of which corresponds to the Onsager phenomenological coefficients. Figure 3.2 depicts the correlations for eutectic composition $\left(x_{\mathrm{KCl}}=0.42\right)$ at two different temperatures, $773 \mathrm{~K}$ and $1200 \mathrm{~K}$, respectively. 


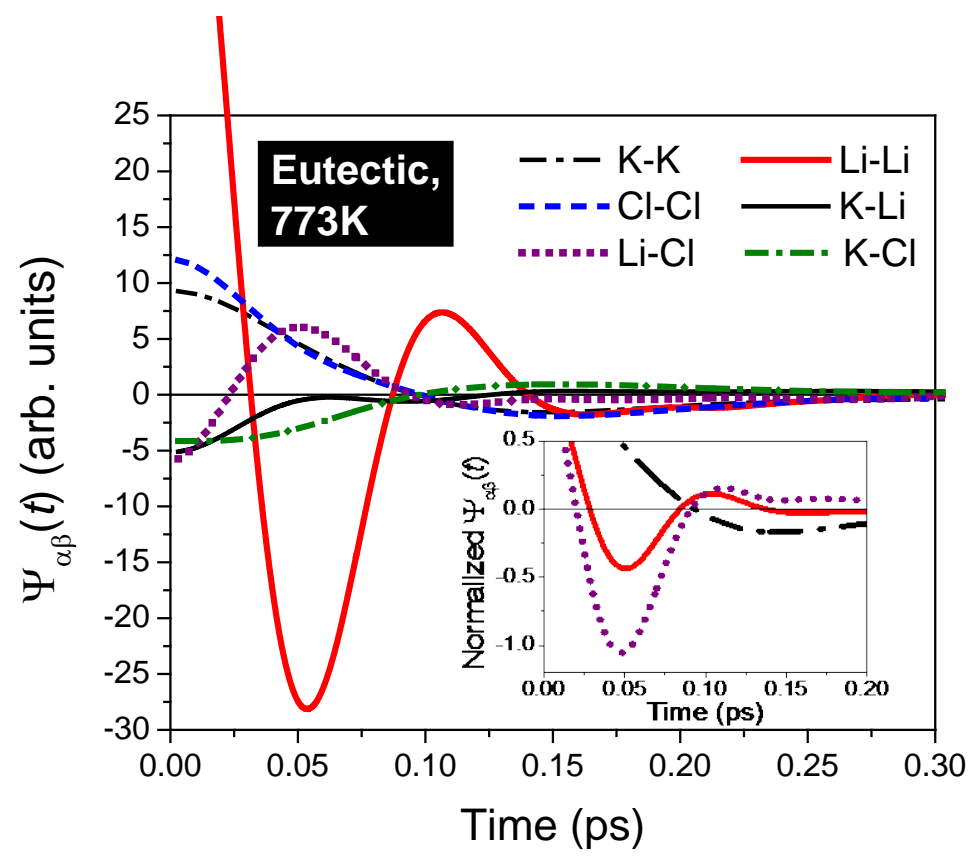

(a)

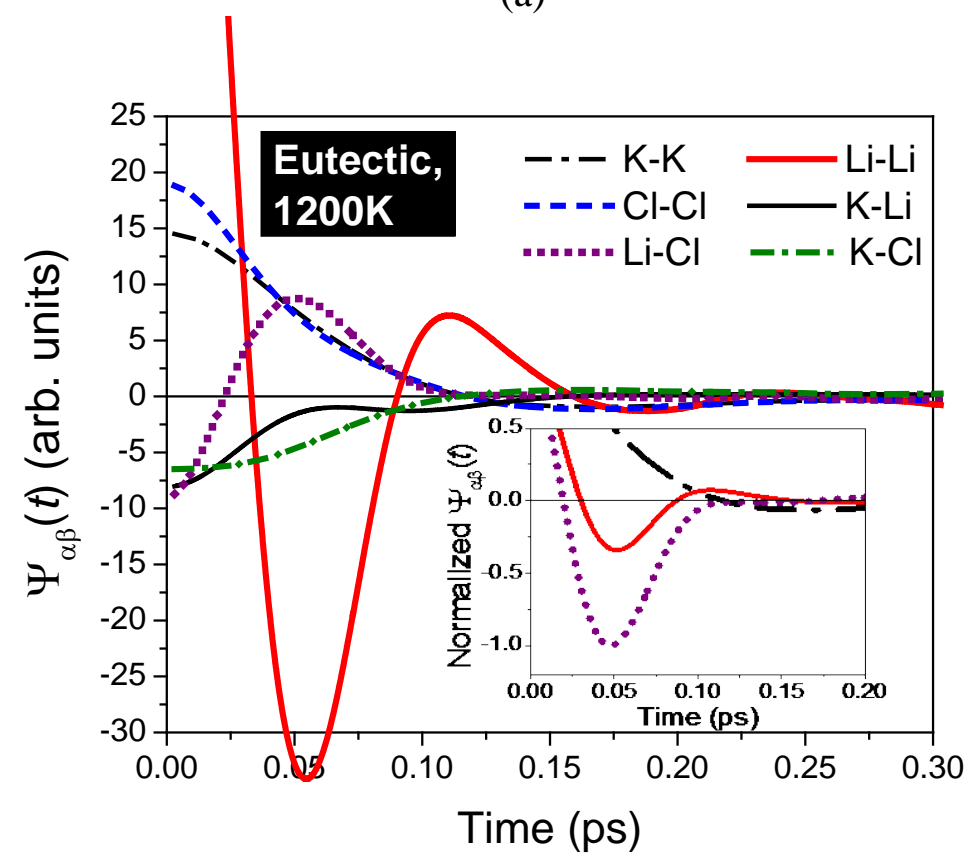

(b)

Figure 3.2. (a) Temporal variation of diffusive flux correlation, $\Psi(t)$ at $773 \mathrm{~K}$. Insets show the normalized diffusive flux correlations for $\mathrm{Li}-\mathrm{Li}, \mathrm{Li}-\mathrm{Cl}$ and $\mathrm{K}-\mathrm{K}$; (b) Temporal variation of diffusive flux correlation, $\Psi(t)$ at $1200 \mathrm{~K}$. Insets show the normalized diffusive flux correlations for $\mathrm{Li}-\mathrm{Li}, \mathrm{Li}-\mathrm{Cl}$ and $\mathrm{K}-\mathrm{K}$.

At a temperature of $773 \mathrm{~K}$ which is approximately $147 \mathrm{~K}$ above the melting point of LiCl-KCl mixture (top panel), all the correlations portray backscattering or cage effect (alternating negative and positive flux correlations) after the initial decay. The strongest backscattering is observed for the $\mathrm{Li}-\mathrm{Li}$ correlation followed by the $\mathrm{Li}-\mathrm{Cl}$ correlation. The correlations for $\mathrm{K}-\mathrm{K}$ and $\mathrm{Cl}-\mathrm{Cl}$ decay almost identically (except at very short times) leading to similar Onsager coefficients. While the 
cage effect diminishes with increasing temperature for $\mathrm{K}-\mathrm{K}$ and $\mathrm{Cl}-\mathrm{Cl}$ correlations (bottom panel), which is expected as higher thermal energy can typically aid in overpowering any entrapment, the correlations for $\mathrm{Li}-\mathrm{Li}$ and $\mathrm{Li}-\mathrm{Cl}$ however, show an interesting endurance of the oscillations.

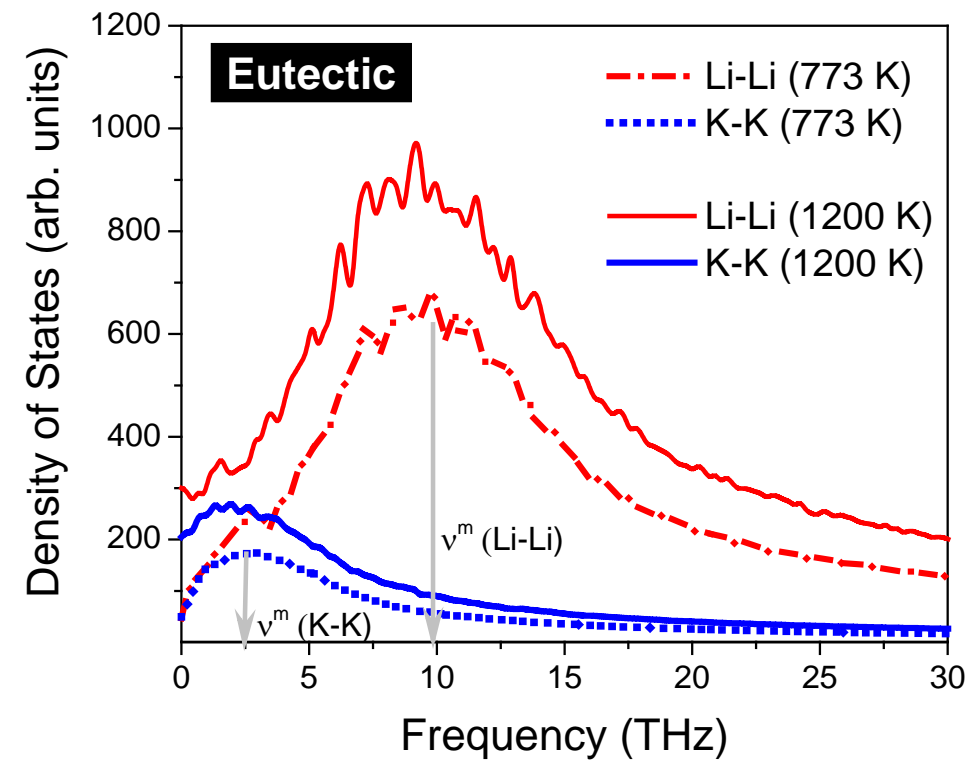

Figure 3.3. Density of States for Li-Li and K-K correlations at $773 \mathrm{~K}$ and $1200 \mathrm{~K}$.

The density of states (DoS) shown in Figure 3.3, which is calculated from the magnitude of the Fourier transform of the diffusive flux correlations, shows that there is a smearing of the peak frequency $\left(v^{\mathrm{m}}=2.5 \mathrm{THz}\right.$, approximately, at $\left.773 \mathrm{~K}\right)$ and a broadening of the low frequencies with increasing temperature for the $\mathrm{K}-\mathrm{K}$ correlation which is consistent with the weakening of the cage dynamics. In contrast, the peak form for the Li-Li correlation remains largely unchanged. Thus the well preserved peak for the $\mathrm{Li}-\mathrm{Li}$ correlation along with only a minor strengthening of the low frequency modes further attest to the persistence of $\mathrm{Li}-\mathrm{Li}$ cage dynamics across the temperatures. A possible reason for this behavior can stem from the intermediate short-ranged order observed in $\mathrm{LiCl}-\mathrm{KCl}$ and other alkali halides mixtures [66]. The short-ranged order in Li-Li is manifested by the presence of a pre-peak at a wave vector in the vicinity of $1 \AA^{-1}$ with the position and width of the pre-peaks unaltered by changes in temperature [66]. The pre-peak is attributed to an incomplete mixing of $\mathrm{LiCl}$ and $\mathrm{KCl}$ at intermediate length scales that promote a homogeneous but disordered $\mathrm{K}-\mathrm{Cl}$ matrix with embedded clusters of Li ions. Thus the Li ions, even though they interact weakly with each other (see Figure 3.1) and have a lower mass relative to the other ions, form a temperature independent, long-lived metastable cage where they bounce back and forth which is manifested as a prolonged and persistent backscattering in the diffusive flux correlations. 


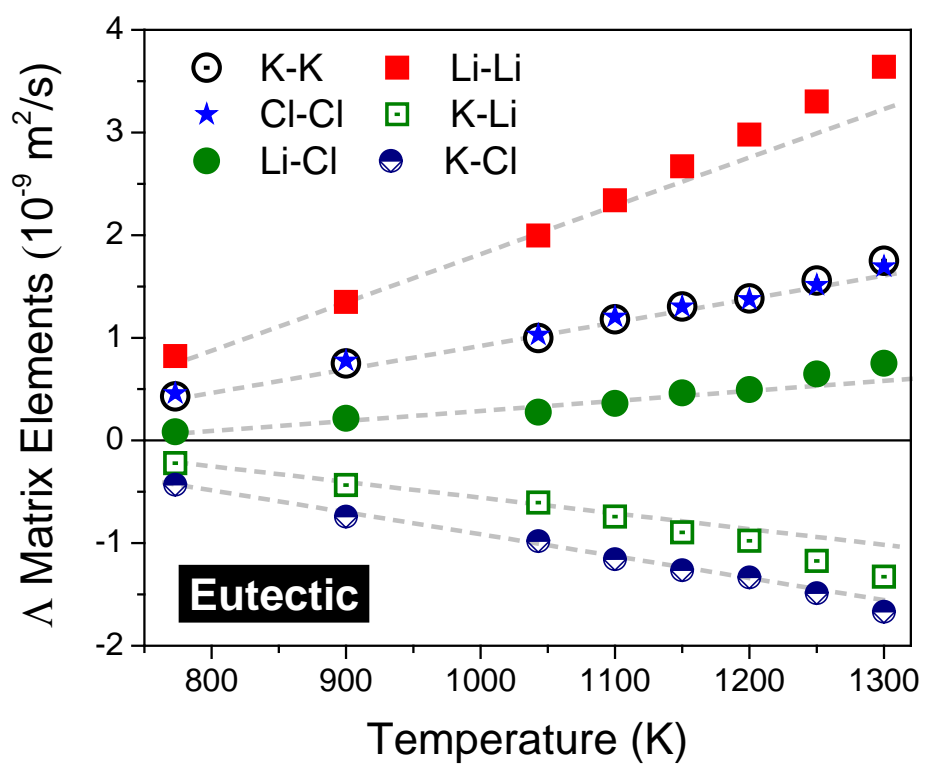

Figure 3.4. Onsager coefficients ( $\Lambda$ matrix elements) at eutectic composition for different temperatures. The broken straight lines serve as guide to the eye.

We will now discuss the temperature variation of the Onsager coefficients which are the time integrals of the diffusive flux correlations as given in Eqn. (3.2.6). Figure 3.4 depicts the six independent Onsager coefficients evaluated with the formally equivalent Einstein form given in Eqn. (3.2.7). Each Onsager coefficient, as stated before, is averaged over 24,000-36,000 independent sets of data. Numerically, the convergence of the Einstein form is observed to be less susceptible to statistical noise unlike the Green-Kubo form in which the flux correlations portray persistent tail fluctuations that impede accurate evaluation of the integrand of the correlation functions. The Einstein form quickly converges to a linear variation in time from which accurate estimates of the slopes [see Eqn. (3.2.7)] can be estimated within 5 ps. As observed from the figure, the Onsager coefficients vary almost linearly with temperature even though a small but systematic change in the slope is noticeable at a temperature of 1100K. Not surprisingly, Li-Li correlation have the highest magnitude followed by the $\mathrm{K}-\mathrm{K}$ and $\mathrm{Cl}-\mathrm{Cl}$ correlations which are nearly identical to each other, as well as the $\mathrm{K}-\mathrm{Cl}$ correlation which is negative at all temperatures. It may be noted that all the Onsager coefficients are $O\left(10^{-9}\right) \mathrm{m}^{2} / \mathrm{s}$ which is expected for typical ions in the liquid state. As shown next, MS diffusivity for the like-like ions can show very large deviation at certain critical temperatures and compositions. 


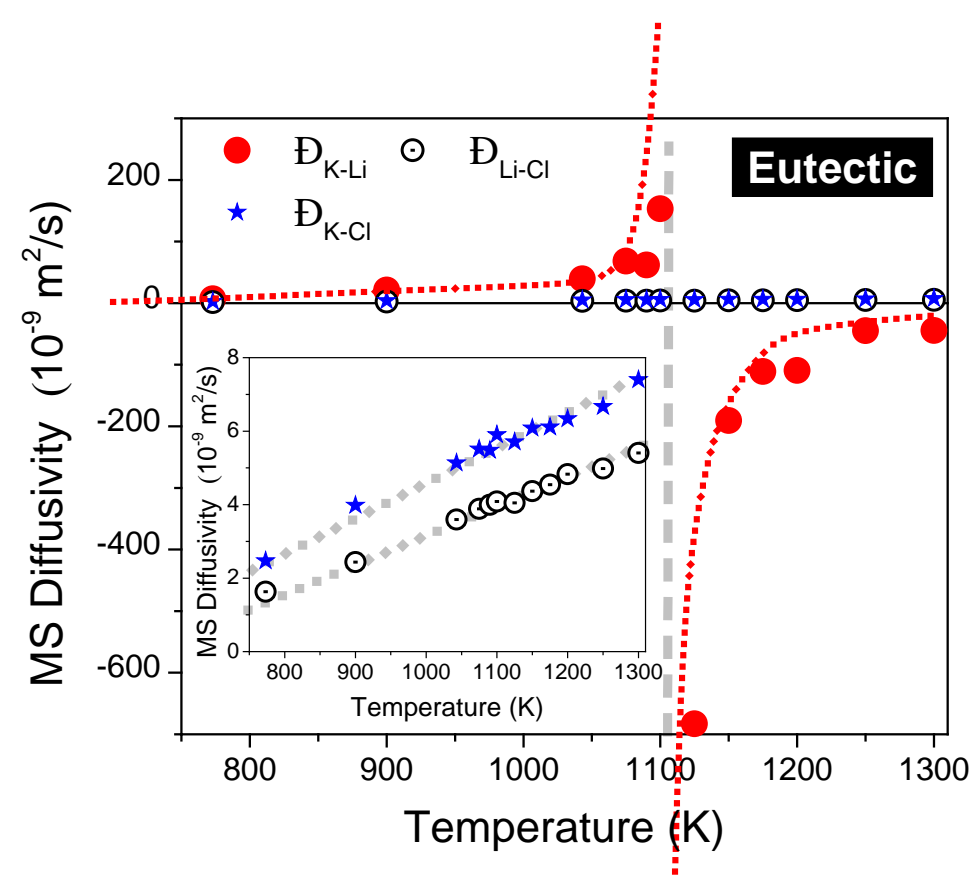

Figure 3.5. MS diffusivities for different temperatures at eutectic composition. (Inset) $\bigoplus_{\mathrm{Li}-\mathrm{Cl}}$ and $\bigoplus_{\mathrm{K}-\mathrm{Cl}}$ on a magnified scale.

Figure 3.5 delineates the variation of MS diffusivities with temperature at eutectic composition. The increase of $\bigoplus_{\mathrm{K}-\mathrm{Li}}$ with increasing temperature followed by a rapid drop and recovery near a temperature of $1100 \mathrm{~K}$ is the most arresting aspect of Figure 3.5. In contrast, $\bigoplus_{\mathrm{Li}-\mathrm{Cl}}$ and $\mathrm{Ð}_{\mathrm{K}-\mathrm{Cl}}$ are rather well-behaved showing a near linear increase over the temperature range (see inset) with a discernible but small kink at $1100 \mathrm{~K}$. Negative and divergent MS diffusivities are theoretically possible when the two terms of the denominators in the MS diffusivity expressions [see Eqn. (3.2.18)] become close to each other. From the variation observed for $\bigoplus_{\mathrm{K}-\mathrm{Li}}$ the temperature which corresponds to diverging $\bigoplus_{\mathrm{K}-\mathrm{Li}}$ is estimated to be $1100 \mathrm{~K}$ (shown by vertical thick broken line). We have further verified that the computed MS diffusivities are consistent with a positive entropy production rate (per unit volume) as portrayed in Figure 3.6. We have further verified that entropy remains non-negative even when $\bigoplus_{\mathrm{K}-\mathrm{Li}}$ is allowed to take any values in the $(+\infty,-\infty)$ range excluding zero within machine precision.

In Figure 3.7 we show the MS diffusivities as a function of composition at $1043 \mathrm{~K}$. As observed with the variation for temperature, the MS diffusivity for the $\mathrm{K}-\mathrm{Li}$ ion pair tends to portray a diverging behavior at a $\mathrm{KCl}$ mole fraction of 0.48 , approximately. The variation of $\bigoplus_{\mathrm{K}-\mathrm{Li}}$ is strikingly similar to those observed in experiments; the inset shows a MS diffusivity data set reconstructed from experimental Fickian diffusion coefficients for like-like ions in an ion exchange membrane system [62]. We have again verified that entropy production rate remains non-negative at all compositions (results not shown). 


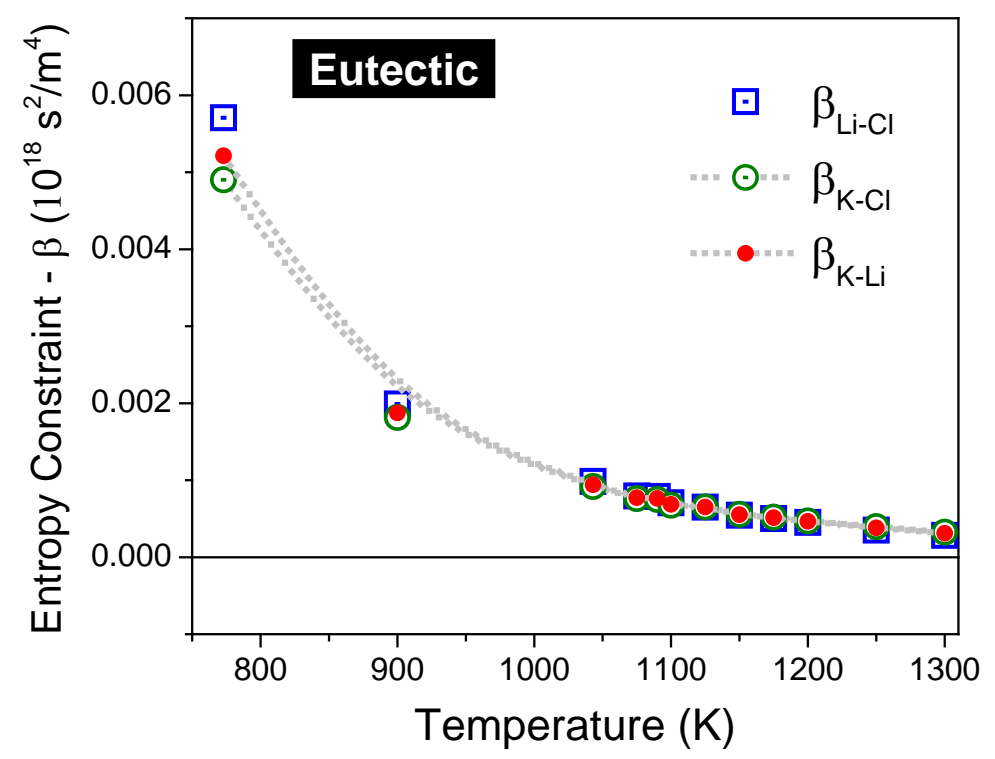

Figure 3.6. Entropy constraint [Eqn. (3.2.20)] showing a non-negative entropy production rate.

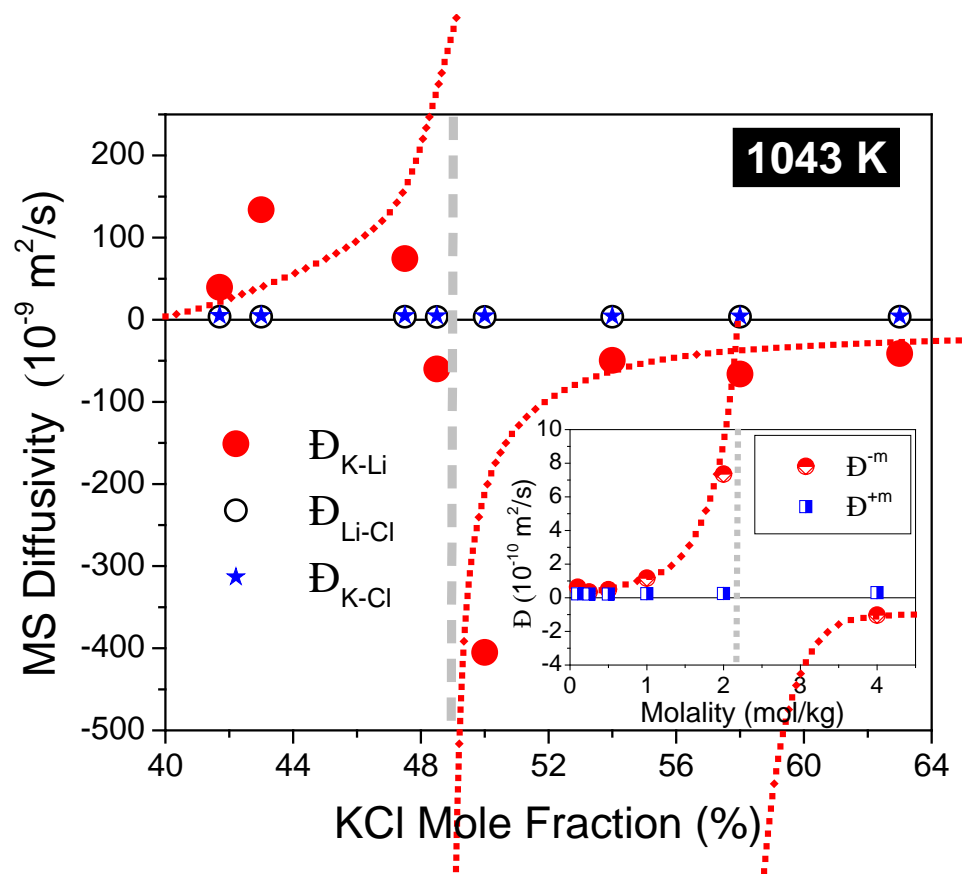

Figure 3.7. MS diffusivities for different compositions at $1043 \mathrm{~K}$. (Inset) Experimental MS diffusivities in an ion exchange membrane (data from [62]). 


\subsubsection{Concluding Remarks}

While experiments and simulations [50, 56, 57] do confirm that MS diffusivities are composition dependent, the current investigation shows that they can also be temperature dependent. Thus, in general, MS diffusivities can be regarded to be strongly dependent on the thermodynamic state which somewhat negates the original motivation for developing the multicomponent MS framework [71]. Further investigations are needed on strongly non-ideal systems to assess the postulated benefits of MS framework in experimental and theoretical analyses. Negative MS diffusivities are somewhat counter-intuitive in the sense that diffusion takes place with a negative friction coefficient. Nevertheless, as observed in the current investigation and in several experiments with electrolytic systems, negative as well as diverging MS diffusivities are within the constraints of the II law of thermodynamics. While negative MS diffusivities occur only for like-like ions in the simulations, as in experiments, a rational physical basis for the negative coefficients has been elusive [72]. To our knowledge the divergence of MS diffusivities has also not gained much attention. From the definition given in Eqn. (3.2.11), a single negative or divergent MS diffusivity does not imply vanishing gradients in the chemical potential. It would however, be interesting to investigate whether a diverging MS diffusivity corresponds to the critical point of the mixture [43] which is also dependent on the thermodynamic state of the system. In principle, the thermodynamic matrix and the non-ideality factors can be evaluated using the KB theory [58] and molecular simulations, even though the evaluation of KB integrals of highly non-ideal, multicomponent systems is non-trivial [57].

\subsubsection{Recommendations}

Given the possibility of encountering the mixture critical point and/or diverging MS diffusivities, it is recommended that users of eutectic salts be aware that some changes in thermophysical properties associated with this behavior might occur at temperatures around 1100K. 


\subsection{Free Energy Simulations Development/Analysis}

In this investigation, we show that the absolute thermodynamic properties of $\mathrm{LiCl} / \mathrm{KCl}$ molten salts with and without actinides can be accurately determined from the 2PT method. The 2PT free energy is in excellent agreement with that determined from the thermodynamic integration (TI) method, with relative errors of $1 \%$ or less, typically. The melting point of ionic $\mathrm{KCl}(1025 \mathrm{~K})$ compares favorably with the experimental data $(1041 \mathrm{~K})$; the entropy of the melt phase also show good conformity with the recommended data from NIST. We then determine the absolute free energy and entropy which are currently unknown for a eutectic mixture of $\mathrm{LiCl}$ and $\mathrm{KCl}$ for temperatures ranging from $773 \mathrm{~K}$ to $1300 \mathrm{~K}$. From the partitioning of the DoS, which is uniquely possible in the 2PT method, we show that the $\mathrm{Li}^{+}$ions in the LiCL-KCl mixture are dominantly solid-like even at temperatures as high as $1300 \mathrm{~K}$. For chemical and nuclear applications the solid-like states can potentially impose practical limits, for example, to the amount of dissolved species that can be practically accommodated during reprocessing. Finally, we evaluate the free energies of dissolved $\mathrm{U}, \mathrm{La}$ and $\mathrm{Sr}$ in Eutectic LiCl-KCl mixtures.

\subsubsection{The Two-Phase Thermodynamic (2PT) Method}

The thermodynamic properties in the canonical ensemble can be evaluated from the canonical partition function $(Q)$; for example, the internal energy $(E)$, entropy $(S)$, specific heat at constant volume $\left(C_{v}\right)$, and the Helmoholtz free energy $(A)$ can be evaluated as [73]

$$
\begin{aligned}
& E=k_{B} T^{2}\left[\frac{\partial}{\partial T}(\ln Q)\right] \\
& S=k_{B}\left[T \frac{\partial}{\partial T}(\ln Q)+\ln Q\right] \\
& C_{v}=\frac{\partial}{\partial T}\left\{k_{B} T^{2}\left[\frac{\partial}{\partial T}(\ln Q)\right]\right\}
\end{aligned}
$$

$$
A=-k_{B} T(\ln Q)
$$

where $k_{B}$ is the Boltzmann constant and $T$ is the absolute temperature. The thermodynamic properties are seldom evaluated through the partition function as it is exactly known only for idealized systems such as harmonic oscillators and ideal gas. Using a normal mode analysis, a solid state can be approximated as a system of non-interacting harmonic oscillators (HO), which in this case the total canonical partition function $\left(Q^{\mathrm{HO}}\right)$ can be expressed as [74]

$Q^{\mathrm{HO}}=\prod_{i=1}^{3 N} q_{i}$ 
where $q_{i}$ is the partition function of the $i^{\text {th }}$ harmonic oscillator mode, and $N$ is the total number of oscillators. The total partition function can be shown to be related to the density of states as [74]

$\ln \left(Q^{\mathrm{HO}}\right)=\int_{0}^{\infty} d v G(v) \ln [q(v)]$

where $G(v)$ is the density of states, which is given by [26]

$G(v)=\frac{4}{k_{B} T} \int_{0}^{\infty} d t \Psi(t) e^{-i 2 \pi v t}$

where $\Psi(t)$ is the mass-weighted velocity autocorrelation function, [73] which can be directly computed from a molecular dynamics (MD) simulation (ab-initio or classical). Since an exact quantum-mechanical expression is known for $q(v)$, the total partition function can be evaluated using Eqs. (3.3.3) and (3.3.4), and the thermodynamic properties such as given by Eqs. (3.3.1a) to (3.3.1d) can be assessed for the solid states with reasonable accuracy [74]. For a liquid state, the system cannot be considered as a collection of harmonic oscillators and a direct application of the above approximation will lead to non-physical results. The 2PT method [26] extends the above idea to the liquid state by assuming a superposition of two idealized states - hard spheres (HS) that correspond to a gas-like state, and harmonic oscillators that correspond to a solid-like state. First it is hypothesized that the total density of states of the system, which is now regarded to be a noninteracting mixture of hard spheres and harmonic oscillators, can be decomposed as

$G_{k}(v)=G_{k}^{\mathrm{HS}}(v)+G_{k}^{\mathrm{HO}}(v)$

where $k$ denotes the different species in the mixture. A key step in the 2PT method is in defining and evaluating a fluidicity parameter $(f)$ given by the following expression for partitioning the system into the idealized states.

$f_{k}=\frac{\int_{0}^{\infty} d v G_{k}^{\mathrm{HS}}(v)}{\int_{0}^{\infty} d v G_{k}(v)}=\frac{3 N_{k}^{\mathrm{HS}}}{3 N_{k}}=\frac{N_{k}^{\mathrm{HS}}}{N_{k}}$

where $N_{k}^{\text {HS }}$ is the effective number of hard sphere atoms or ions of the $k^{\text {th }}$ component of the mixture. Note that the integral of the total density of states over all frequencies is simply $3 N_{k}$. Thus the $3 N_{k}$ degrees of freedom of the $k^{\text {th }}$ component is assumed to be composed of $3 N_{k}^{\text {HS }}$ or equivalently, $3 f_{k} N_{k}$ hard sphere or diffusive degrees of freedom, and $3\left(N_{k}-N_{k}^{\mathrm{HS}}\right)$ or equivalently, $3 N_{k}\left(1-f_{k}\right)$ solid-like or non-diffusive degrees of freedom [29]. With this approximation, the thermodynamic properties are formally expressed as [29]

$$
E_{k}=E_{k}^{0}+k_{B} T\left[\int_{0}^{\infty} d v G_{k}^{\mathrm{HS}}(v) W_{E_{k}}^{\mathrm{HS}}(v)+\int_{0}^{\infty} d v G_{k}^{\mathrm{HO}}(v) W_{E}^{\mathrm{HO}}(v)\right]
$$




$$
\begin{aligned}
& S_{k}=k_{B}\left[\int_{0}^{\infty} d v G_{k}^{\mathrm{HS}}(v) W_{S_{k}}^{\mathrm{HS}}(v)+\int_{0}^{\infty} d v G_{k}^{\mathrm{HO}}(v) W_{E}^{\mathrm{HO}}(v)\right] \\
& C_{v, k}=k_{B}\left[\int_{0}^{\infty} d v G_{k}^{\mathrm{HS}}(v) W_{C_{v, k}}^{\mathrm{HS}}(v)+\int_{0}^{\infty} d v G_{k}^{\mathrm{HO}}(v) W_{C_{v}}^{\mathrm{HO}}(v)\right] \\
& A_{k}=E_{k}^{0}+k_{B} T\left[\int_{0}^{\infty} d v G_{k}^{\mathrm{HS}}(v) W_{A_{k}}^{\mathrm{HS}}(v)+\int_{0}^{\infty} d v G_{k}^{\mathrm{HO}}(v) W_{A}^{\mathrm{HO}}(v)\right]
\end{aligned}
$$

where $W$ is a weight function for the appropriate property, and $E_{k}^{0}$ is the reference energy which is given by [29]

$$
E_{k}^{0}=E_{k}^{\mathrm{MD}}-3 N_{k} k_{B} T\left(1-\frac{f_{k}}{2}\right)
$$

where $E_{k}^{\mathrm{MD}}$ is the total energy from MD simulations for the $k^{\text {th }}$ component. Thus each extensive thermodynamic property, for each mixture component, comprises of two contributions - one from diffusive (hard spheres) degrees of freedom, and the other from vibrating (harmonic oscillator) degrees of freedom. The distinguishing part of the 2PT method is in deriving an exact expression for $G_{k}^{\text {HS }}$ given by [26]

$$
G_{k}^{\mathrm{HS}}(v)=G_{k}(0)\left[1+\left(\frac{\pi G_{k}(0) v}{6 f_{k} N_{k}}\right)^{2}\right]^{-1}
$$

Thus the density of states for the hard sphere phase can be calculated as a function of the fluidicity parameter and $G_{k}(0)$ which is just the zero-frequency value of the system (total) density of states. MD simulations are employed to calculate $G_{k}(v)$ from the velocity autocorrelation function. With a known $G_{k}^{\mathrm{HS}}$, the density of states of the HO phase is then evaluated as $G_{k}^{\mathrm{HO}}(v)=\left[G_{k}(v)-G_{k}^{\mathrm{HS}}(v)\right]$. The weight functions are known exactly for the HO and HS idealized states, and are given by [13, 29]

$$
\begin{aligned}
& W_{E}^{\mathrm{HO}}=\frac{\beta h v}{2}+\frac{\beta h v}{e^{\beta h v}-1} \\
& W_{S}^{\mathrm{HO}}=\frac{\beta h v}{e^{\beta h v}-1}-\ln \left(1-e^{-\beta h v}\right)
\end{aligned}
$$

$$
W_{C_{v}}^{\mathrm{HO}}=\frac{(\beta h v)^{2} e^{\beta h v}}{\left(e^{\beta h v}-1\right)^{2}}
$$




$$
\begin{aligned}
& W_{A}^{\mathrm{HO}}(v)=\ln \left[\frac{\left(1-e^{-\beta h \nu}\right)}{e^{-\beta h \nu / 2}}\right] \\
& W_{E_{k}}^{\mathrm{HS}}(v)=W_{C_{v, k}}^{\mathrm{HS}}(v)=\frac{1}{2} \\
& W_{S_{k}}^{\mathrm{HS}}(v)=\frac{S_{k}^{\mathrm{HS}}(v)}{3 k_{B}} \\
& W_{A_{k}}^{\mathrm{HS}}(v)=\left[W_{E_{k}}^{\mathrm{HS}}(v)-W_{S_{k}}^{\mathrm{HS}}(v)\right]=\left(\frac{1}{2}-\frac{s_{k}^{\mathrm{HS}}(v)}{3 k_{B}}\right)
\end{aligned}
$$

where $\beta^{-1}=k_{B} T, h$ is the Planck constant and $s^{\mathrm{HS}}$ is the entropy of the hard sphere phase (per atom/ion), which in the 2PT model is evaluated as

$$
s_{k}^{\mathrm{HS}}=s_{k}^{\mathrm{IG}}+k_{B}\left\{\ln \left[z_{k}\left(\hat{y}_{k}\right)\right]+\frac{\hat{y}_{k}\left(3 \hat{y}_{k}-4\right)}{\left(1-\hat{y}_{k}\right)^{2}}\right\}
$$

The first term of the above equation denotes the ideal gas entropy, however, weighted only for the HS phase. It is given by [13]:

$$
s_{k}^{\mathrm{IG}}=k_{B}\left\{\frac{5}{2}+\ln \left[\left(\frac{2 \pi m_{k} k_{B} T}{h^{2}}\right)^{3 / 2}\left(\frac{V_{k}}{f_{k} N_{k}}\right)\right]\right\}
$$

where $m_{k}$ and $N_{k}$ are the mass and the total number of ions/atoms of the $k^{\text {th }}$ species, respectively. The term $z_{k} \equiv P V_{k} /\left(N_{k} k_{B} T\right)$ in Eqn. (3.3.12) delineates the compressibility of the hard sphere phase, which is determined from the Carnahan-Starling equation given by

$$
z_{k}\left(\hat{y}_{k}\right)=\frac{1+\hat{y}_{k}+\hat{y}_{k}^{2}-\hat{y}_{k}^{3}}{\left(1-\hat{y}_{k}\right)^{3}}
$$

where $\hat{y}_{k}$ is the weighted hard sphere packing fraction (by the fluidicity parameter) for the $k^{\text {th }}$ component, and is given by $\hat{y}_{k}=f_{k} y_{k}$ where $y_{k}$ denotes the packing fraction which is expressed as

$$
y_{k}=\frac{\pi \rho_{k}\left(\sigma_{k}^{H S}\right)^{3}}{6}
$$

where $\rho_{k}$ is the number density and $\sigma_{k}^{H S}$ is the hard sphere diameter of the $k^{\text {th }}$ species that in turn influences the fluidicity parameter $\left(f_{k}\right)$. In the 2PT methodology it is assumed that the fluidicity 
parameter is simply a ratio of the self diffusivity $\left(D_{k}\right)$ to the hard sphere diffusivity at zero pressure $\left(D_{k}^{\bullet H S}\right)$, which can be written as

$$
f=\frac{D(T, P)}{D_{k}^{\bullet H S}\left(T, P ; \sigma^{H S}\right)}
$$

The self-diffusivity can be evaluated from MD simulations as the time integral of the velocity autocorrelation, and is expressible as

$D_{k}(T, P)=\frac{k_{B} T G_{k}(0)}{12 m_{k} N_{k}}$

where $G_{k}(0)$ is evaluated from the expression

$G_{k}(v)=\frac{4}{k_{B} T} \int_{0}^{\infty} d t \Psi_{k}(t) e^{-i 2 \pi v t}$

We invoke the stationary principle of classical correlation functions [75], and thus only the real part of the Fourier transform needs to be used for calculating the integral in the previous equation. Note that $\Psi(t)$ is the mass-weighted velocity autocorrelation function defined as

$$
\Psi_{k}(t)=m_{k} \sum_{i=1}^{N_{k}} \int_{0}^{\infty} d t\left\langle\mathbf{v}_{k}(t) \cdot \mathbf{v}_{k}(0)\right\rangle=m_{k} \sum_{i=1}^{N_{k}} \int_{0}^{\infty} d t\left\langle\psi_{k}(t)\right\rangle
$$

where $\psi_{k}(t)$ is the velocity autocorrelation function of the $k^{\text {th }}$ component. The hard sphere diffusivity in the limit of zero pressure/density is computed exactly from the Chapman-Enskog's theory, which is given by

$$
D_{k}^{\bullet H S}\left(T, P ; \sigma_{k}^{H S}\right)=\frac{3}{8 \rho_{k}\left(\sigma_{k}^{H S}\right)^{2}}\left(\frac{k_{B} T}{\pi m_{k}}\right)^{1 / 2}
$$

The velocity autocorrelation for HS phase decays exponentially in time and hence the HS diffusivity can be expressed as [26]

$$
D_{k}^{H S}\left(T, f_{k} \rho_{k}\right)=\frac{k_{B} T G_{k}(0)}{12 m_{k} f_{k} N_{k}}
$$

The above expression can be related to the zero-pressure hard sphere diffusivity through the following relationship

$$
D_{k}^{H S}\left(T, f_{k} \rho_{k}\right)=D_{k}^{\bullet H S}\left(T, f_{k} \rho_{k} ; \sigma_{k}^{H S}\right) \frac{4 \hat{y}_{k}}{\left[z\left(\hat{y}_{k}\right)-1\right]}
$$


where $\hat{y}_{k}=f_{k} y$. After a few algebraic steps the following relationship can be established [29]

$2 \Delta_{k}^{-9 / 2} f_{k}^{15 / 2}-6 \Delta_{k}^{-3} f_{k}^{5}-\Delta_{k}^{-3 / 2} f_{k}^{7 / 2}+6 \Delta_{k}^{-3 / 2} f_{k}^{5 / 2}+2 f_{k}-2=0$

where

$\Delta_{k}=\frac{2 G_{k}(0)}{9 N_{k}}\left(\frac{\pi k_{B} T}{m_{k}}\right)^{1 / 2}\left(\frac{N_{k}}{V_{k}}\right)^{1 / 3}\left(\frac{6}{\pi}\right)^{2 / 3}$

Equation (3.3.24) contains the details of the physical state as well as the zero-frequency density of states that can be evaluated from MD simulations. Eqn. (3.3.23) now can be iteratively solved to get the fluidicity parameter $\left(f_{k}\right)$ for each component of the mixture. Once $f$ is known, the density of states and the weight functions of the HS phase can be computed, and the HO density of states can be determined as $G_{k}^{\mathrm{HO}}=\left(G_{k}-G_{k}^{\mathrm{HS}}\right)$. Thus, all the properties given by Eqs. (3.3.7a) to (3.3.7d) can be determined, for each component of the mixture.

\subsubsection{Partial Volumes and Mixture Properties}

In this investigation, the partial volume $\left(V_{k}\right)$ in Eqn. (3.3.24) is calculated from the relative ionic radii, which is as given by

$V_{k}=\frac{\sigma_{k}^{3}}{\sum_{k=1}^{n} x_{k} \sigma_{k}^{3}}\left(\frac{V}{N}\right)$

where $\sigma_{k}$ and $x_{k}$ are the ionic radius and mole fraction, respectively, of the $k^{\text {th }}$ component. The Kirkwood and Buff (KB) theory [58] gives a better approximation for partial volumes but it entails the evaluation of somewhat ill-convergent KB integrals. We have used a fixed ionic radius of 0.152 $\mathrm{nm}, 0.167 \mathrm{~nm}$ and $0.09 \mathrm{~nm}$ for $\mathrm{K}^{+}, \mathrm{Cl}^{-}$and $\mathrm{Li}^{+}$ions, respectively [76]. As shown later, the ionic radius approximation works quite well for the current application. Finally, the molar $(m)$ thermodynamic properties of the mixture are determined as

$$
\begin{aligned}
& \bar{E}^{m}=\sum_{k=1}^{n} x_{k} E_{k}^{m} \\
& \bar{S}^{m}=\sum_{k=1}^{n} x_{k} S_{k}^{m}-\hat{R} \sum_{k=1}^{n} x_{k} \ln \left(x_{k}\right) \\
& \bar{A}^{m}=\sum_{k=1}^{n} x_{k} A_{k}^{m}+\hat{R} T \sum_{k=1}^{n} x_{k} \ln \left(x_{k}\right)
\end{aligned}
$$

where $\hat{R}$ is the universal gas constant, and $n$ is the total number of components in the mixture. 


\subsubsection{Molecular Dynamics Simulations}

MD simulations are principally employed for calculating the velocity autocorrelation function. The molecular system typically consists of 1000 ions interacting through a long-ranged electrostatic potential and a Born-Huggins-Mayer (BHM) short-ranged potential. The MD simulations are performed using the LAMMPS software [77] with periodic boundary conditions at zero pressure using a timestep of $1 \mathrm{fs}$. Particle-particle-particle-mesh is used for columbic interactions, and the temperature and pressure are controlled using the Nose-Hoover algorithm. From the velocity data collected from MD simulations, the velocity autocorrelation function (VACF) is constructed, typically with a correlation time of 25 ps, using an overlapped data structure [70]. From the Fourier transform of the VACF, the 2PT thermodynamic properties are computed using an in-house computer program.

\subsubsection{Results and Discussion}

We will first discuss (1) the numerical convergence of the fluidicity parameter with correlation time, followed by (2) a benchmark investigation of 2PT method against published results from thermodynamic integration (TI) method for $\mathrm{KCl}$, (3) computation of the molar free energy and the entropy change across melting in $\mathrm{KCl}$ along with a comparison to NIST data[78], and (4) an assessment of the density of states and thermodynamic properties of a eutectic mixture of $\mathrm{LiCl}-\mathrm{KCl}$ for different temperatures.

\subsubsection{Numerical convergence of the fluidicity parameter}

The fluidicity parameter for each component of the mixture is iteratively solved using Eqn. (3.3.23). The partitioning of the system into solid-like and gas-like states depends on resolving the zerofrequency value of the density of states. To assess the optimal correlation time (for VACF), we have first computed $f_{k}$ using Eqn. (3.3.23) and then compare with the reconstructed $\hat{f}_{k}$ from the equation

$$
\hat{f}_{k}=\frac{\int_{0}^{\infty} d v G_{k}^{\mathrm{HS}}(v)}{\int_{0}^{\infty} d v G_{k}(v)}=\frac{1}{3 N_{k}} \int_{0}^{\infty} d v G_{k}^{\mathrm{HS}}(v)
$$




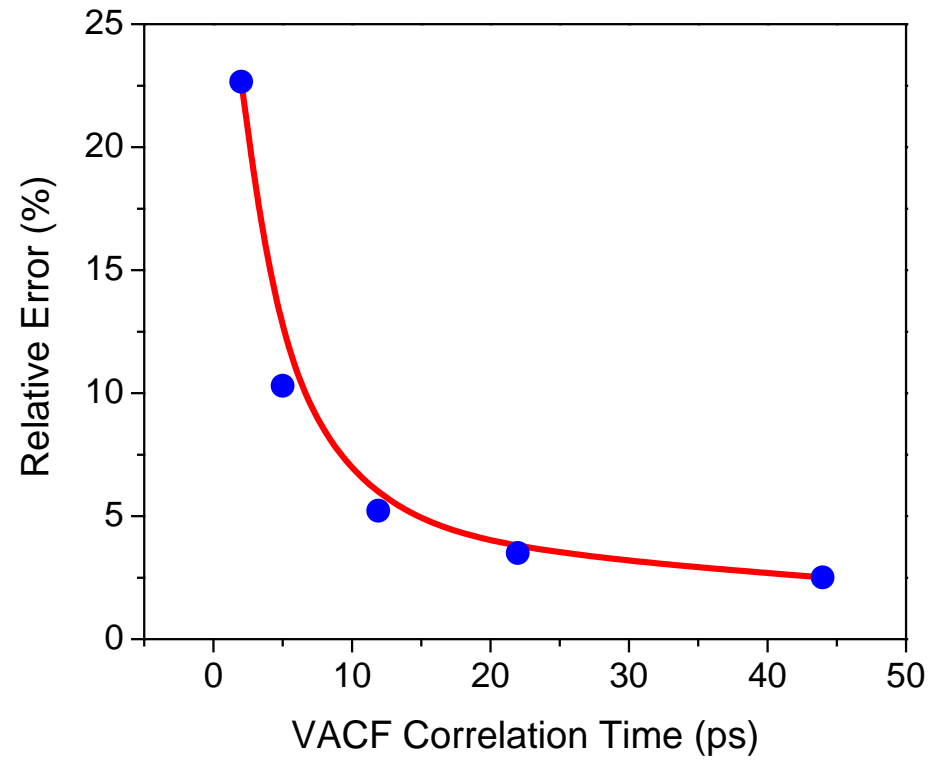

Figure 3.8. Relative error in reconstructing the fluidicity parameter for the $\mathrm{K}^{+}$ions in molten $\mathrm{KCl}$.

Insufficient sampling and numerical errors in the evaluation of VACF and the density of states will be reflected as a difference between $\hat{f}_{k}$ and $f_{k}$. Figure 3.8 shows the error in the reconstruction, expressed as a percentage, for the $\mathrm{K}^{+}$ions in molten $\mathrm{KCl}$ at a temperature of $1100 \mathrm{~K}$ and at a density of $20 \mathrm{~nm}^{-3}$. At short correlation times that are less than $5 \mathrm{ps}$, the numerical errors are significant; they however, reduce almost exponentially with increasing correlation time. It is interesting to note that the rate of decrease of the fluidicity parameter is considerably weaker beyond $\sim 20 \mathrm{ps}$; in our simulations, we have used a fixed correlation time of 25 ps, which generally results in a reconstruction error of 3 to $4 \%$.

\subsubsection{Benchmarking of 2PT free energy with thermodynamic integration (TI) method}

We will now turn to benchmarking the predictions from the 2PT method with published data on free energy of molten $\mathrm{KCl}$ determined from the thermodynamic integration (TI) method. Absolute free energy has been computed by Rodrigues and Fernandes using a coupling TI method using the BHM potential [18]. The Einstein crystal method is used to compute the absolute free energy of the solid state, while a two-step procedure is employed for the liquid state. First the BHM potential is converted to a half-wing repulsive Gaussian potential with the repulsive barrier kept close to that of the original potential. Next the Gaussian potential is converted into a null potential to transition into the ideal gas reference state with known thermodynamic properties. For the liquid states, checks have been made to ensure that the TI paths are reversible and no phase change occurs along them [18]. 


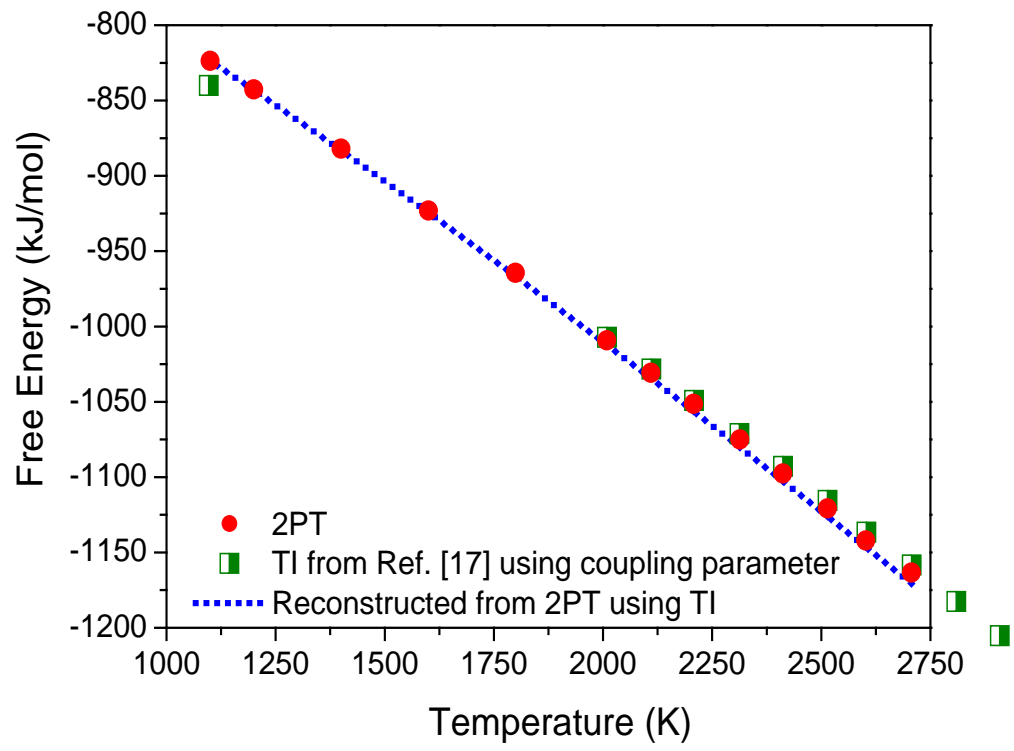

Figure 3.9. Comparison of absolute (molar) free energy of molten $\mathrm{KCl}$ from the 2PT and $\mathrm{TI}$ [18] methods at a temperature of $1100 \mathrm{~K}$ and a density of $20 \mathrm{~nm}^{-3}$.

The free energy computed by the 2PT method (circles) and coupling TI method [18] (squares) shown in Figure 3.9confirm the accuracy of the 2PT method against a well-established method; the relative error between the predictions is typically less than 1\%. Unlike the coupling TI method, the 2PT method requires only modest computing time to derive the free energy with comparable precision. Also shown in the figure is the reconstructed free energy (dotted line) from a thermodynamic integration approach using the internal energy computed from the 2PT method [13]. First the entropy at a certain temperature $\left(T_{2}\right)$ is computed as

$$
S_{k}\left(T_{2}\right)=S_{k}\left(T_{1}\right)+\int_{T_{1}}^{T_{2}} d T \frac{1}{T}\left(\frac{d E_{k}}{d T}\right)_{2 P T}
$$

where $E_{k}$ is the internal energy determined from the 2PT method as shown in Eqn. (3.3.7a) for the $k^{\text {th }}$ component of the mixture. The internal energy is next linearly fitted to temperature which gives a constant derivative for $E_{k}$ (the specific heat at constant volume). Then Eqn. 3.3.28 is integrated (numerically or exactly) to evaluate the entropy at different states, followed by the reconstruction of the free energy as $A_{k}=E_{k}-T S_{k}$. As evident from Figure 3.9, the reconstructed free energy is in close agreement with the original estimate; somewhat better agreement can be obtained from a higher order fit to the energy variation with temperature. 


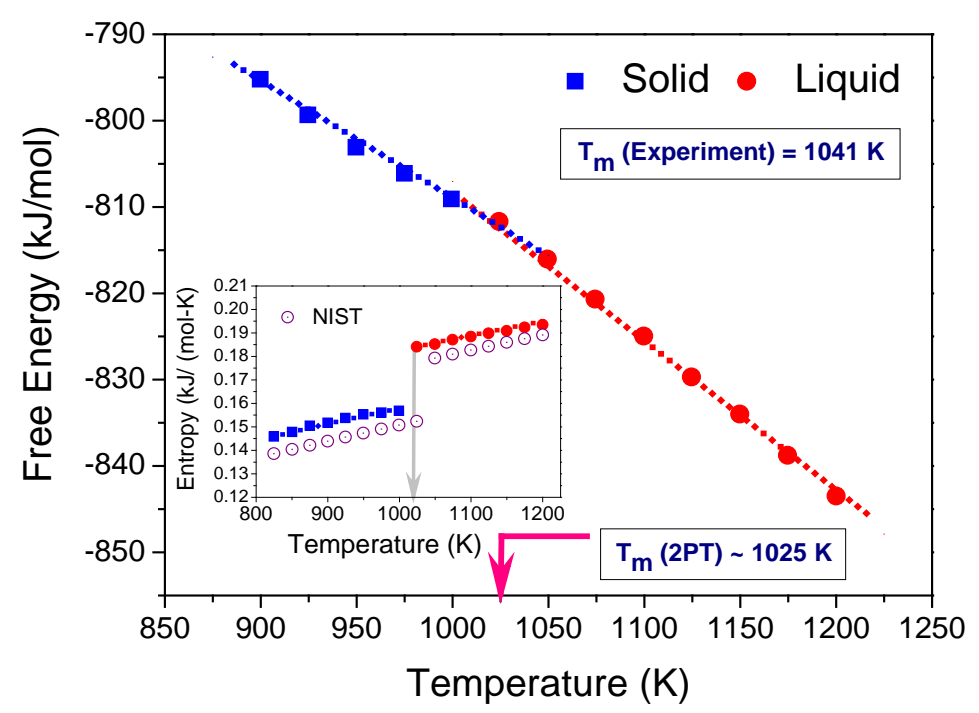

Figure 3.10. Molar free energy change across melting of $\mathrm{KCl}$ with the 2PT method. (Inset) Entropy jump from 2PT and comparison to NIST standard entropy data (open circles) [78].

A first-order phase transition occurs at the thermodynamic melting point, which is exemplified by a slope change for the free energy along with a discontinuity in properties such as entropy. In Figure 3.10, the free energies of the solid and liquid phases are plotted as a function of temperature $-\mathrm{a}$ discernible change in the slope occurs at $1025 \mathrm{~K}$, which corresponds to the melting point of $\mathrm{KCl}$. This 2PT estimate compares favorably with the experimental melting point of $1041 \mathrm{~K}$ with a relative error of $1.5 \%$, approximately. It may be noted that the fluidicity parameter for the solid states are negligible.

The melting point is also measurable from other properties from a MD simulation such as from the change in the structure function; the 2PT method, however, is particularly advantageous as it can compute the properties such as entropy and specific heat which undergo a discontinuity across the phase change. The inset shows an entropy jump of $0.0273 \mathrm{~kJ} /(\mathrm{mol}-\mathrm{K})$ across melting which agrees very well with the NIST data of $0.0269 \mathrm{~kJ} /(\mathrm{mol}-\mathrm{K})$ [78] at standard conditions, incurring a relative error of $1.5 \%$. However, there is a systematic over-prediction by the 2PT method leading to a relative error of 2-3\% between the absolute entropy values for the liquid states and somewhat higher for the solid states; the difference can partly arise from the inaccuracy of the interionic potential. The dotted lines in the inset are the reconstructed entropy values by integrating Eqn. (3.3.28) - the close agreement again highlights the self-consistency of the 2PT methodology.

\subsubsection{Molar free energy and entropy of eutectic $\mathrm{LiCl}-\mathrm{KCl}$ mixture}

Lithium ions in the LiCl-KCl mixtures behave very differently from the potassium or chlorine ions; earlier discussion shows that the molar flux correlations for the $\mathrm{Li}^{+}$group $\left(\mathrm{Li}^{+}-\mathrm{Li}^{+}, \mathrm{Li}^{+}-\mathrm{Cl}^{-}\right.$and $\mathrm{Li}^{+}-\mathrm{K}^{+}$) behave very differently from the $\mathrm{K}^{+}$group [79]. Of particular interest is the formation of long lived dynamical cage for the $\mathrm{Li}^{+}-\mathrm{Li}^{+}$and $\mathrm{Li}^{+}-\mathrm{Cl}^{-}$interactions, even at high temperatures, 
which has been ascribed to the intermediate-ranged ordering of the $\mathrm{Li}^{+}$ions [35, 66]. An incomplete mixing of $\mathrm{LiCl}$ and $\mathrm{KCl}$ has been put forward as a plausible mechanism for the intermediate-ranged ordering, which manifests as a pre-peak in the structure function at $1 \AA$, approximately. In the current study, we investigate the density of states, particularly the partitioning into solid-like and gas-like components, and probe the dynamical characteristics that determine the thermodynamic properties.
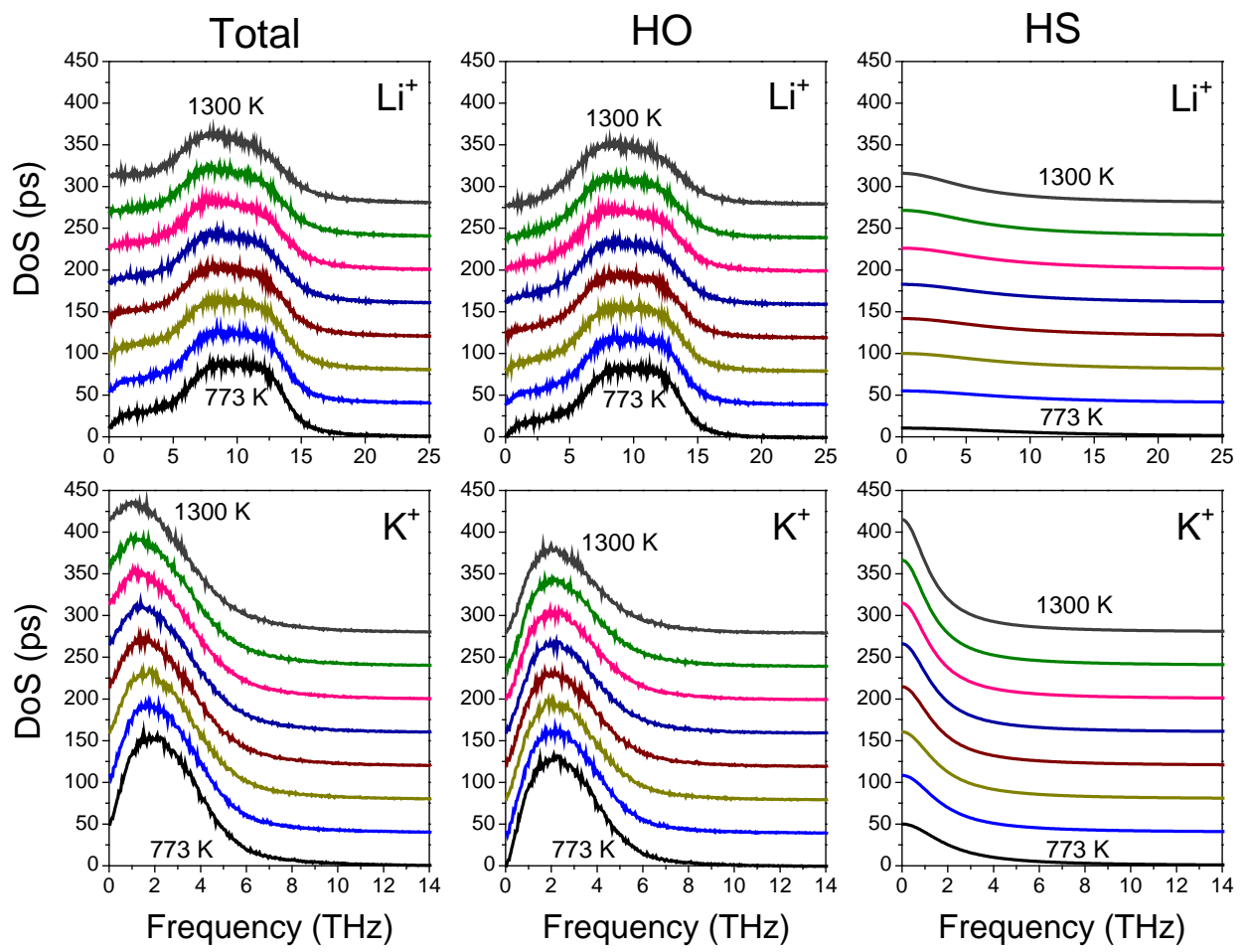

Figure 3.11. Density of states (DoS): total (left column), solid-like (HO, middle column), and gas-like (HS, right column) for $\mathrm{Li}^{+}$(top row) and $\mathrm{K}^{+}$ions (bottom row) at different temperatures (773 K to $1300 \mathrm{~K}$ ). The DoS plots for different temperatures - $773 \mathrm{~K}, 850 \mathrm{~K}, 950 \mathrm{~K}, 1043 \mathrm{~K}, 1100 \mathrm{~K}, 1150 \mathrm{~K}, 1200 \mathrm{~K}, 1300 \mathrm{~K}$ ) - are off-set by 40 ps for visual clarity.

The total DoS for the $\mathrm{K}^{+}$ions is similar to that observed in typical liquids with a discernible zerofrequency component that augments with increasing temperature, and a single peak at a frequency that is $\mathrm{O}(1) \mathrm{THz}$. In contrast the total DoS for the $\mathrm{Li}^{+}$ions portrays a plateau-like peak region spanning a broad range of frequencies, which are well-preserved even at high temperatures. Interestingly, the magnitude of the total $\mathrm{DoS}$ for the $\mathrm{Li}^{+}$ions is almost zero indicating a solid-like behavior, which is further affirmed by the DoS partitions - the HO contribution practically accounts for the total DoS with negligible contribution from the hard spheres. The $\mathrm{Li}^{+}$behavior is consistent with the persistent and prolonged backscattering observed in the molar flux correlations [79], as well as the presence of the intermediate-ranged ordering [66]. The total DoS for the $\mathrm{K}^{+}$ions, on the other hand, is dominated by the contribution from the harmonic oscillators at low temperatures; as temperature increases, the contribution from the hard spheres also increases significantly. Interestingly, the total and partial DoS characteristics for the $\mathrm{Cl}^{-}$ions are very similar 
to those of the $\mathrm{K}^{+}$ions; however, with a more prominent contribution from the hard sphere phase. Thus in the molten LiCl-KCl state, even at high temperatures, $\mathrm{Li}^{+}$ions depict a predominantly solidlike characteristic, while the $\mathrm{K}^{+}$and $\mathrm{Cl}^{-}$ions portray a more conventional fluid-like behavior with a significant contribution from the hard sphere phase.

Next we show the free energy and entropy of LiCl-KCl mixture at eutectic composition for different temperatures in Figure 3.12 (for 1 mole of $\mathrm{LiCl}$ and 1 mole of $\mathrm{KCl}$ ). With the addition of $\mathrm{LiCl}$ to $\mathrm{KCl}$, the melting point reduces from $1043 \mathrm{~K}$ and attains the lowest value of $626 \mathrm{~K}$ at the eutectic composition $\left(x_{\mathrm{KCL}}=0.42\right)$. As expected the entropy increases with increasing temperature and shows a somewhat linear behavior from $\sim 1050 \mathrm{~K}$, which perhaps, is related to the occurrence of melting of pure $\mathrm{KCl}$ around this temperature $(1041 \mathrm{~K})$; the results in the previous section also show an instability in the $\mathrm{K}^{+}-\mathrm{Li}^{+}$Maxwell-Stefan (MS) diffusivity at $1100 \mathrm{~K}$ [79]. Further investigations are needed to ascertain the physical significance, if any, for this behavior.

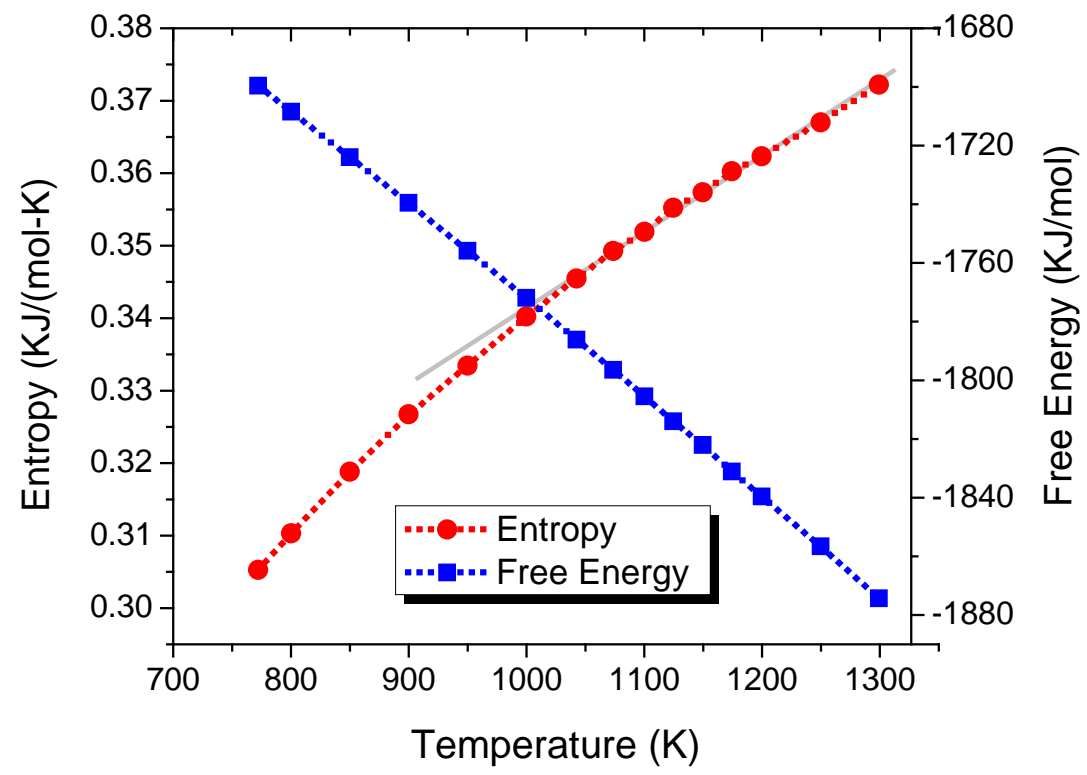

Figure 3.12. Free energy/entropy for LiCl-KCl mixture at eutectic composition $\left(x_{\mathrm{KCL}}=0.42\right)$.

\subsubsection{Concluding Remarks}

The 2PT method is a robust and accurate methodology for determining absolute thermodynamic properties of fluids and fluid mixtures. We have applied this method to calculate the absolute free energy and entropy of molten $\mathrm{KCl}$, and $\mathrm{LiCl}-\mathrm{KCl}$ mixture at eutectic composition. The 2PT predictions show excellent agreement with those from a coupling thermodynamic integration (TI) method; for comparable states, the relative error is typically less than $1 \%$. Further the 2PT method accurately predicts the melting point of molten $\mathrm{KCl}$ and the entropy change across melting with errors not exceeding few percents.

The 2PT method is less reliant on prior judgment - as pointed out [17], the TI method generally benefits from the judicious choice of potential parameters/optimal paths for determining the 
absolute properties, which are not always known, a priori. As shown in this investigation, the 2PT method is robust and can handle complex liquids and mixtures with relative ease. These desirable features make the 2PT method very appealing for chemical and nuclear reprocessing applications where the processes entail a large number of dissolved species. Further there is a satisfying physical basis for the 2PT method, which allows the extraction of dynamical characteristics that determine the thermodynamic properties; as elucidated before, the 2PT method is unique in this aspect.

\subsubsection{Recommendations}

We have shown that the $\mathrm{Li}^{+}$ions in molten $\mathrm{LiCl}-\mathrm{KCl}$ have very dominating solid-like and practically negligible fluid-like characteristics, unlike the $\mathrm{K}^{+}$and $\mathrm{Cl}^{-}$ions. This could have practical implications, in particular, it is easier for the dissolved species in the presence of solid-like ions to change the solvent characteristics, say to highly viscous, glassy states [25]. Thus there may be a trade-off in having a eutectic mixture of LiCl-KCl - on one hand, a significantly lower melting point can be achieved that is of immense value from a practical point of view, while on the other, the presence of solid-like ions, particularly close to the melting point, may not be optimum for reprocessing and waste recovery applications. 


\subsection{Prediction of Properties with Sr/La/U solute}

In this section, we apply the 2PT method to the eutectic $\mathrm{LiCl}-\mathrm{KCl}$ mixture with $\mathrm{Sr}^{2+}$ ions ( $\mathrm{Sr}$ solute), $\mathrm{La}^{3+}$ ions (La solute) and $\mathrm{U}^{3+}$ ions (U solute). For the Sr solute, a BHM potential is used with parameters developed at the University of Wisconsin. For the La and U solute systems, we have chosen the potential developed by Salanne et al. [4] without the polarization term, which is not considered significant. A temperature of $773 \mathrm{~K}$ is chosen for the systems, which is the typical reprocessing temperature. For the $\mathrm{Sr} / \mathrm{LiCl} / \mathrm{KCl}$ mixture, a total of 990 ions are used with $280 \mathrm{Li}^{+}$ ions, $200 \mathrm{~K}^{+}$ions, $500 \mathrm{Cl}^{-}$ions and $10 \mathrm{Sr}^{2+}$ ions, while for the $\mathrm{U} / \mathrm{LiCl} / \mathrm{KCl}$ mixture, 980 ions are employed with $274 \mathrm{Li}^{+}$ions, $196 \mathrm{~K}^{+}$ions, $500 \mathrm{Cl}^{-}$ions and $10 \mathrm{U}^{3+}$ ions. For the La solute ions in the LiCl-KCl mixture, 980 ions are employed with $274 \mathrm{Li}^{+}$ions, $196 \mathrm{~K}^{+}$ions, $500 \mathrm{Cl}^{-}$ions and $10 \mathrm{La}^{3+}$ ions. The ionic radius for $\mathrm{Sr}^{2+}$ and $\mathrm{U}^{3+}$ ions are $0.132 \mathrm{~nm}$ and $0.1165 \mathrm{~nm}$, respectively [76]. Due to the small number of solute ions, 20 independent runs are employed to obtain a respectable statistical average for mass-weighted VACF/DoS.

\subsubsection{Sr Solute}

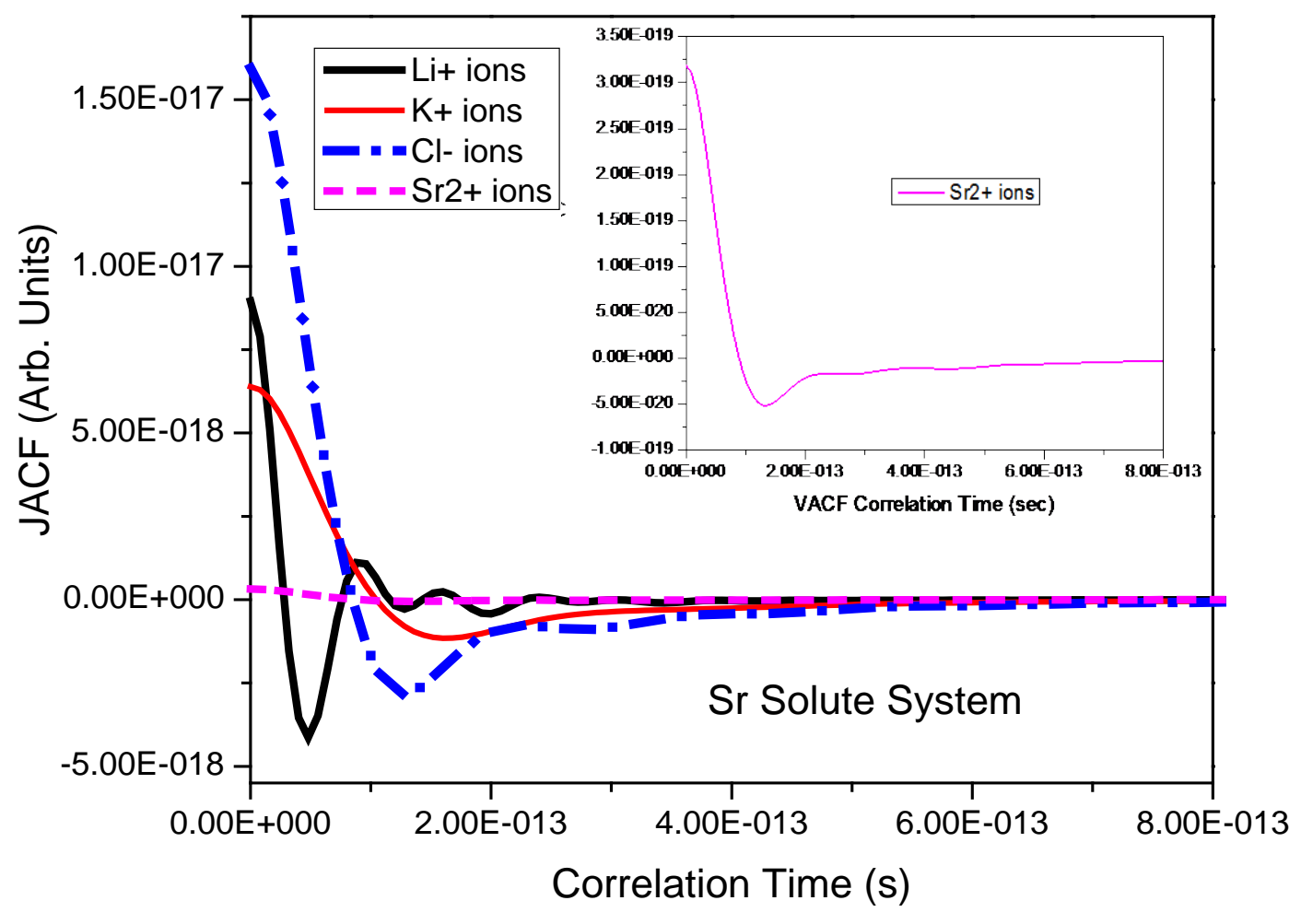

Figure 3.13. Mass-weighted velocity autocorrelation (JACF) for the Sr solute system. (Inset) JACF for $\mathrm{Sr}^{2+}$ ions. A total of 990 ions are used with $280 \mathrm{Li}^{+}$ions, $200 \mathrm{~K}^{+}$ions, $500 \mathrm{Cl}^{-}$ions and $10 \mathrm{Sr}^{2+}$ ions. 
The mass weighted velocity autocorrelation (JACF) for each species in the Sr solute system is shown in Figure 3.13. The density of states (DoS) of the Sr solute system at $773 \mathrm{~K}$ and 0 pressure is shown in Figure 3.14. As expected, the solid-like component for $\mathrm{Li}$ ions is small relative to $\mathrm{K}$ and $\mathrm{Cl}$ ions. Interestingly, the DoS for Sr ions has a significant solid-like component, which outweighs the HS gas-like component. Thus we show that Sr ions, even at very low number/volume fraction, have a significant solid-like dynamic response. In Table 3.1, we depict the absolute thermodynamic properties of the Sr solute system.
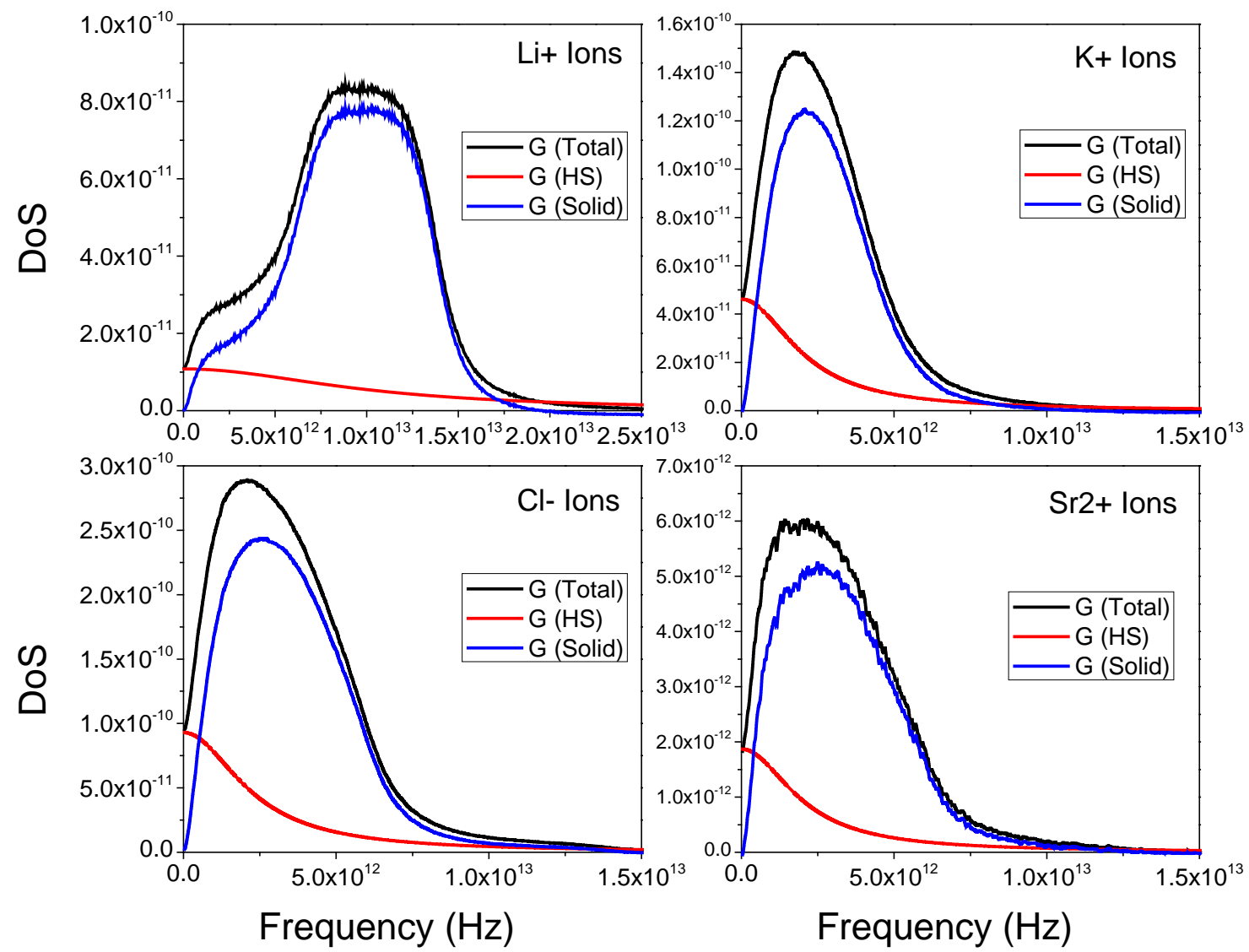

Figure 3.14. The density of states (DoS) of the Sr solute system at $773 \mathrm{~K}$ and 0 pressure. The total DoS $(G)$ is shown in black, the hard sphere phase (HS) in red and the harmonic oscillator (Solid) in blue. A total of 990 ions are used with $280 \mathrm{Li}^{+}$ions, $200 \mathrm{~K}^{+}$ions, $500 \mathrm{Cl}^{-}$ions and $10 \mathrm{Sr}^{2+}$ ions. 
Table 3.1. The 2PT results of Sr solute system at $773 \mathrm{~K}$ and $0 \mathrm{~Pa}$. A total of 990 ions are used with $280 \mathrm{Li}^{+}$ ions, $200 \mathrm{~K}^{+}$ions, $500 \mathrm{Cl}^{-}$ions and $10 \mathrm{Sr}^{2+}$ ions.

\begin{tabular}{|c|c|c|c|c|c|}
\hline Species & $\mathrm{Li}^{+}$ & $\mathrm{K}+$ & $\mathrm{Cl}-$ & $\mathrm{Sr}^{2+}$ & Sr mixture \\
\hline$E(\mathrm{~kJ} / \mathrm{mol})$ & -416.692 & -218.158 & -362.625 & -1178.09 & -356.898 \\
\hline$S(\mathrm{~kJ} / \mathrm{mol}-\mathrm{K})$ & 0.0466 & 0.0796 & 0.0743 & 0.0759 & 0.0764 \\
\hline$A(\mathrm{~kJ} / \mathrm{mol})$ & -452.721 & -279.689 & -420.004 & -1236.75 & -415.969 \\
\hline
\end{tabular}

E: Total energy, S: Entropy, A: Free energy

\subsubsection{La Solute}

In Table 3.2, the absolute thermodynamic properties for the La solute system are delineated. A total of 980 ions are employed with $274 \mathrm{Li}^{+}$ions, $196 \mathrm{~K}^{+}$ions, $500 \mathrm{Cl}^{-}$ions and $10 \mathrm{La}^{3+}$ ions.

Table 3.2. The 2PT results of La solute system at $773 \mathrm{~K}$ and $0 \mathrm{~Pa}$.

\begin{tabular}{|c|c|c|c|c|c|}
\hline Species & $\mathrm{Li}+$ & $\mathrm{K}+$ & $\mathrm{Cl}-$ & $\mathrm{La}^{3+}$ & La mixture \\
\hline$E(\mathrm{~kJ} / \mathrm{mol})$ & -86.234 & -591.321 & -253.024 & -2534.624 & -297.321 \\
\hline$S(\mathrm{~kJ} / \mathrm{mol}-\mathrm{K})$ & 0.028 & 0.049 & 0.043 & 0.053 & 0.049 \\
\hline$A(\mathrm{~kJ} / \mathrm{mol})$ & -107.593 & -629.303 & -286.515 & -2575.665 & -334.739 \\
\hline
\end{tabular}

\subsubsection{U Solute}

In Table 3.3, the absolute thermodynamic properties for the U solute system are delineated. A total of 980 ions are employed with $274 \mathrm{Li}^{+}$ions, $196 \mathrm{~K}^{+}$ions, $500 \mathrm{Cl}^{-}$ions and $10 \mathrm{U}^{3+}$ ions.

Table 3.3. The 2PT results of U solute system at $773 \mathrm{~K}$ and $0 \mathrm{~Pa}$.

\begin{tabular}{|c|c|c|c|c|c|}
\hline Species & $\mathrm{Li}+$ & $\mathrm{K}+$ & $\mathrm{Cl}-$ & $\mathrm{U}^{3+}$ & $\mathrm{U}$ mixture \\
\hline$E(\mathrm{~kJ} / \mathrm{mol})$ & -422.517 & -121.56 & -252.595 & -2541.09 & -297.241 \\
\hline$S(\mathrm{~kJ} / \mathrm{mol}-\mathrm{K})$ & 0.0278 & 0.049 & 0.043 & 0.061 & 0.050 \\
\hline$A(\mathrm{~kJ} / \mathrm{mol})$ & -443.934 & -159.435 & -286.131 & -2588.48 & -334.738 \\
\hline
\end{tabular}

\subsubsection{Recommendations}

A small number/volume fraction of actinides generally results in augmenting the solid-like dynamic characteristics of the base LiCl-KCl mixture. Since Li ions also have a dominant solid-like response, it is possible that pure $\mathrm{KCl}$ will be more suitable at higher temperatures for actinide recovery. 


\section{Technical Report from the University of Wisconsin-Madison (Task 1 \& 2)}

Researchers: Amelia Bengtson, Hyo On Nam, Saumitra Saha, Ridwan Sakidja, Katharina Vörtler and Dane Morgan

This section reports on results from Tasks 1 and 2

\subsection{Introduction}

In this research, we firstly explore the applicability of first-principles molecular dynamics (FPMD) simulations to the prediction of properties of $\mathrm{LiCl}-\mathrm{KCl}$ molten salt system with various compositions (mole fraction of $\mathrm{LiCl}=1,0.58$, and 0 ). In particular we seek to obtain converged liquids with stable structure factors from FPMD, calculate volumes, coefficients of thermal expansion, bulk moduli, species diffusion, and the Gibbs free energy of mixing as a function of temperature, and determine if error bars (precision) and accuracy are adequate with present simulation tools to guide experiments.

Measurements on molten salts can be limited by costly and demanding experiments and computational simulations provide a low cost alternative to experiments and can rapidly explore many different temperatures and salt compositions. However, FPMD methods have been applied only rarely to direct calculation of salt properties. Detailed calculations, with careful assessment of uncertainties and comparison to experiments, are needed to establish the optimal approaches and their limitations.

We focus the $\mathrm{LiCl}-\mathrm{KCl}$ as it is a system of considerable interest for the nuclear community with both extensive quality data for validation and yet many open questions that can be investigated with the methods being considered here. Eutectic LiCl-KCl (58\% LiCl, $42 \% \mathrm{KCl}$ ), is a common molten salt used in electrorefining of spent nuclear fuel to separate actinides and other fission products [8083]. While the basic salt properties are known and can be used to validate our approaches, the thermokinetics of solutes within $\mathrm{LiCl}-\mathrm{KCl}$ are not well characterized and can be a subject of future modeling [80, 81, 84-86].

The primary goal of the present work is to validate the practicality of direct FPMD approaches for molten salt studies, which are an increasingly viable alternative to the more commonly used interatomic potential molecular dynamics (IPMD) simulations.

In classical IPMD simulations, Newton's equations of motion are solved in a system where interactions between atoms are described by an interatomic potential, which typically contains the physics of ionic repulsion, attraction, dispersion and polarization. IPMD can be used to predict many properties of salts and their solutes [87-91] and is computationally many orders of magnitude faster than FPMD. However, each new salt and each solute and charge state requires fitting a different potential, which can be time consuming and is subject to the inevitable uncertainty of fitted potentials. Furthermore, such potentials do not typically treat the electronic structure explicitly, and therefore cannot easily be used to track electronic properties (e.g., magnetic 
moments) or redox reactions that might occur between species (although relative stabilities of equivalent impurities have been studied [92]).

FPMD simulations are similar to IPMD in that Newton's equations of motion are also solved, but the atomic interactions are calculated directly from first-principles in FPMD calculations. FPMD therefore requires no fitting to experimental or computed data and provides access to the full electronic structure of the material at every step. The main disadvantage is that FPMD calculations are very computationally intensive and therefore limited to much smaller systems and shorter simulation times than IPMD.

FPMD simulations have successfully modeled liquids such as $\mathrm{CaAl}_{2} \mathrm{O}_{4}$ [93], $\mathrm{CaMgSi}_{2} \mathrm{O}_{6}$ [94], $\mathrm{MgSiO}_{3}$ [95], $\mathrm{Ni}$ alloys [96] and ionic liquids [97]. The $\mathrm{NaCl}$ and $\mathrm{KCl}$ molten salt systems were studied with density-functional-based tight-binding methods (DFT-TB) [98]. While DFT-TB methods are similar to FPMD, they are not fully self-consistent. Furthermore, volumes within the work of Hazebroucq, et al. [98] were fixed to experimental values rather than predicted. FPMD simulations were conducted in the liquid Flibe $\left(\mathrm{Li}_{2} \mathrm{BeF}_{4}\right)$ using the Car-Parrinello method [99], but the focus was primarily on diffusion, again at a fixed experimental volume. These limited studies to date show that these methods can be used effectively, but do not provide an extensive enough study of basic properties to establish optimal approach or assess limitations (e.g., in the ability to predict volumes).

Our research is the first full FPMD study on molten salt systems with fully relaxed volumes. Specifically, we will study the LiCl-KCl system and extend previous FPMD work on molten salt systems [99] to include calculations of equilibrium volume, convergence testing on key parameters as a function of unit cell size and simulation time, statistical errors on all values, and rigorous comparison to experiments, when possible. The LiCl-KCl molten salt system has been studied extensively with classical interatomic potential molecular dynamics [87-91] and this data was used for comparison.

With the developed FPMD approaches, behavior of Sr/La/U solutes, which represent actinides (U), lanthanides ( $\mathrm{La}$ ) and fission product $(\mathrm{Sr})$, in $\mathrm{LiCl}-\mathrm{KCl}$ at $500^{\circ} \mathrm{C}$ is investigated. From the FPMD simulations, local structure surrounding solutes is predicted by analyzing the radial distribution function. And self-diffusion a coefficient of solutes in molten $\mathrm{LiCl}-\mathrm{KCl}$ is calculated from the mean-squared displacement (MSD) analysis as performed in case of constituents of the LiCl-KCl. The redox potential for each element, which is important property in partitioning technology of nuclear wastes, was also predicted from the FPMD simulations. 


\subsection{FPMD Calculations for Pure Li, $\mathrm{KCl}$}

\subsubsection{Details of Computational Methods}

A combination of first-principles molecular dynamics (FPMD) and interatomic potential molecular dynamics (IPMD) was used in this research. All liquids were equilibrated with IPMD and the final converged structure was used to start the FPMD calculation. FPMD calculations starting from the IPMD liquid reach equilibration within 0.3 ps. The technique of starting FPMD calculations from the liquid created with IPMD has been used successfully in the literature $[96,100]$. Note that it might seem to defeat at least part of the purpose of using FPMD if a potential needs to be developed for IPMD to initiate the FPMD calculations. However, while we do not demonstrate it here, it is reasonable to expect the FPMD is quite insensitive to the quality of the IPMD initiation, provided it gets qualitative structural features approximately correct. Thus we expect that no significant time will have to be spent on potential development for IPMD simulations provided they are used for no more than initiating a FPMD simulation.

For the pure $\mathrm{LiCl}$ and $\mathrm{KCl}$, we started simulations with crystalline $\mathrm{LiCl}$ and $\mathrm{KCl}$ structure. In case of the $\mathrm{LiCl}-\mathrm{KCl}$ eutectic mixture $(58 \% \mathrm{LiCl}, 42 \% \mathrm{KCl})$, initial configuration of atoms was created from Packmol [101], a code designed to randomly pack atoms into a given volume. While IPMD was typically initiated with a crystalline structure for $\mathrm{LiCl}$ and $\mathrm{KCl}$, a randomly distributed initialization of the $\mathrm{LiCl}$ system from Packmol (see Computational methods section) also shows a converged liquid structure after the IPMD equilibration and showed same radial distribution functions as initialization from a crystal structure. All IMPD simulations were run with the LAMMPS [102] using the Born-Mayer-Huggins potentials for $\mathrm{LiCl}$ and $\mathrm{KCl}$ [88] with a radial cutoff of approximately half the lattice parameter for a given unit cell $(6,7,9,11,15 \AA$ for 64,100 , 216, 400, and 1000 atom unit-cells). Formal charges were used for Li (+1), Cl (-1), and K (+1). Simulations, unless otherwise noted, contained 216 atoms. The timestep was 0.001 ps and thermal data was outputted every 100 steps.

A series of ensembles was used to equilibrate the liquid structures. The NVE ensemble with velocities generated from random numbers was run for 5,000 steps to bring the system to the correct temperature. Next, a crude Berendsen barostat [103] for 50,000 steps brought the system to the target pressure. Then, simulations with the NPT ensemble (using the Nosé-Hoover thermostat and barostat) were run for 50,000 time steps for pure $\mathrm{LiCl}$ and $\mathrm{KCl}(500,000$ for $\mathrm{LiCl}-\mathrm{KCl})$ to equilibrate system at desired pressure and temperature. The lattice was averaged over last quarter of the NPT time steps and used to start the NVT simulation. The NVT ensemble was run for 100,000 time steps for $\mathrm{LiCl}$ and $\mathrm{KCl}(600,000$ for $\mathrm{LiCl}-\mathrm{KCl})$ for a final equilibration. The resulting structure file was resized for multiple volumes around the experimental volume. Each volume was run with the NVT ensemble for an additional 100,000 time steps. The resulting structure and velocities were used to initialize the VASP NVT simulations.

All FPMD simulations were run with the Vienna Ab-Initio Simulation Package VASP version 5.2.11 [100, 104, 105]. PAW-PBE potentials supplied with the VASP package were used for Li (s1p0 17Jan2003), Cl (s2p5 17Jan2003) and K (p6s1 K_sv 06Sep2000). All simulations were run with the canonical ensemble (NVT) using a Nosé thermostat [106] with a Nosé-mass with a period of 40 time steps. Note that the NPT ensemble is not available for VASP versions earlier than 5.3.2, which was recently released. Energy cutoff of $420 \mathrm{eV}$ and a $1 \times 1 \times 1 \mathrm{k}$-point mesh were used. 
Charges were calculated within the VASP code. A time step of 0.002 ps was used, which gave an energy drift of $<1 \mathrm{meV} / \mathrm{atom} / \mathrm{ps}$, a value similar to that seen in other FPMD simulations [96]. Simulations were run for 3,000 time steps (unless noted). Dispersion was added through the semiempirical DFT-D2 method [107]. The DFT-D method has been shown to work well for ionic liquids [97]. Three compositions are considered within the 64-atom unit cell: $\mathrm{LiCl}$ (32 Li atoms, $32 \mathrm{Cl}$ atoms), $\mathrm{KCl}$ (32 K atoms, $32 \mathrm{Cl}$ atoms) and $\mathrm{LiCl}-\mathrm{KCl}$ near the eutectic composition (19 $\mathrm{Li}$ atoms, $32 \mathrm{Cl}$ atoms, $13 \mathrm{~K}$ atoms; $59.4 \% \mathrm{LiCl})$. Within the 216-atom unit cell, the eutectic composition is $63 \mathrm{Li}$ atoms, $108 \mathrm{Cl}$ atoms, and $45 \mathrm{~K}$ atoms $(58.3 \% \mathrm{LiCl})$.

Periodic boundary conditions were used in both IPMD and FPMD and simulations represent an infinite bulk material. The pressure, temperature, volume and energy of the ensemble are the statistical averages over many time steps. The standard deviation in the mean was calculated from the autocovariance function [108]. To calculate the equilibrium volume with the NVT ensemble, calculations were run at multiple fixed volumes. The resulting pressures, volumes and their associated errors were fit with a Murnaghan equation of state [109] to determine the equilibrium volume and bulk modulus.

The pressure at a finite temperature includes both a kinetic energy and virial force term [110]:

$$
P V=N k_{B} T+\langle W\rangle
$$

VASP (versions prior to 5.3.2) does not automatically include the kinetic energy term in the pressure. The kinetic energy term, $\frac{N k_{B} T}{V}$, was added in post-processing to the pressure for each simulation. This term is usually on the order of 0.35-0.52 GPa in the temperature range 800-1096 K for the $\mathrm{LiCl}-\mathrm{KCl}$ system.

Diffusion is related through the slope of the mean squared displacement (MSD) by the Einstein equation $[110,111]$ :

$$
D=\frac{1}{6} \lim _{t \rightarrow \infty} \frac{d}{d t}(M S D)
$$

In practice, $\mathrm{D}$ was determined by fitting a linear function to $\mathrm{MSD}(\mathrm{t})$ for a subset of the times, as described below. The MSD is determined by a multiple time origin average [111]:

$$
\operatorname{MSD}(t)=\left\langle\Delta r^{2}(t)\right\rangle=\frac{1}{N} \frac{1}{n_{t}} \sum_{j=0}^{n_{t}} \sum_{i=0}^{N}\left(r_{i}\left(t_{0 j}+t\right)-r_{i}\left(t_{0 j}\right)\right)^{2}
$$

where $N$ is the number of atoms in the system, $n_{t}$ is the number of time origins, $t$ is time, and $t_{0 j}$ is the initial timestep originating at time $j[110,111]$.

Unless noted, all diffusion simulations were run with FPMD in VASP at the equilibrium volume with the NVT ensemble and 216 atoms for 12 ps. Mean squared displacements were calculated after the first 200 time steps ( $0.4 \mathrm{ps)}$ to avoid any initial equilibration period. The remaining time steps were divided into four equal blocks and the mean squared displacement was calculated as a function 
of time for each block. In this study, the number of time origins $\left(n_{t}\right)$ is fixed to the half of the block size in order to avoid the noise that is otherwised observed at the last part of time steps. The use of half the block size for $n_{t}$ ensures that each MSD values at the time of $(t)$ are obtained from the same length of trajectory data. The diffusion coefficient is calculated for each block by fitting the slope of the MSD vs. time. To obtain a robust linear region of the MSD data in the block, the first 25 time steps, which often show the expected quadratic MSD(t) dependence [110] are excluded. An error on $\mathrm{D}$ from a given block is then estimated from the fitting error in the slope of MSD vs. time following the statistical equations [112]. The final diffusion coefficient is the average of the diffusion coefficients of each block. The error of each diffusion coefficient from the linear fitting is propagated through the averaging over blocks to the final standard deviation of the mean. This block averaging method is similar to that of Rapaport [113].

One approximation made within density functional theory is the treatment of the exchange correlation between electrons. Common choices include the local density approximation (LDA), which considers only the local electron density, and the Generalized Gradient Approximation with the PBE parameterization (PBE), which considers the gradient of the electron density. Additionally, the PBEsol [114] potential improves upon PBE for solids and is effective for alkali metals and alkali halides.

Differences amongst these exchange-correlation functions are often well known in solids, e.g., GGA typically overestimates volumes and LDA underestimates volumes [115]. These differences are not as well established in high-temperature molten salts. Multiple volumes were run for PBE, PBEsol and LDA in LiCl (64 atoms) and the resulting pressure-volume curves are shown in Figure 4.1. Without dispersion, PBE yields the largest volume, the volume with PBEsol is $~ 5 \%$ less than with PBE, and the volume with LDA is almost $30 \%$ less than with PBE. The inclusion of dispersion corrections (see Computational methods section) lowers the pressure by up to $1 \mathrm{GPa}$. For $\mathrm{PBE}$ and PBEsol, adding dispersion decreases the volume by 15-20 \%. In the LiCl-KCl system, the best match to experimental volumes for all compositions is obtained with the PBE exchange correlations run with the inclusion of dispersion (DFT-D2 method). Therefore, unless noted, all future FPMD calculations in this work are done with PBE and DFT-D2 to include dispersion. 

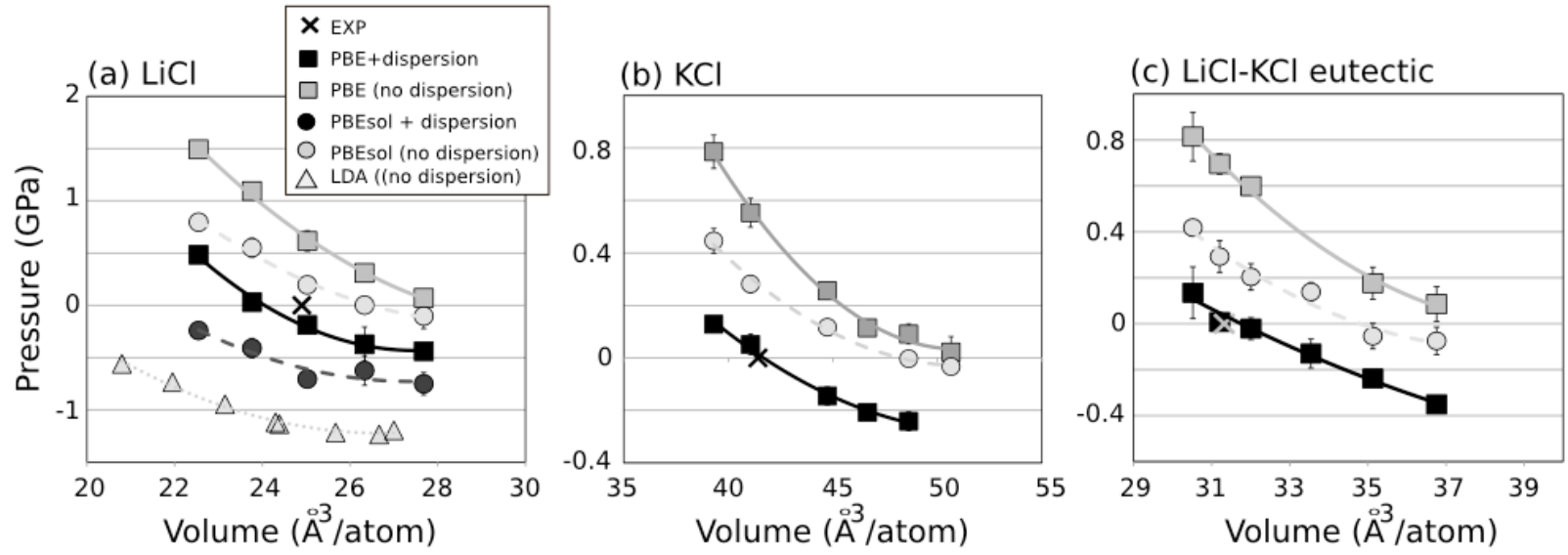

Figure 4.1. Comparison of FPMD computational choices at $1096 \mathrm{~K}$ in VASP. All simulations were run with 64 atoms. PBE, PBEsol and LDA are different choices controlling the exchange correlation within a density functional theory calculation. EXP is experimental data from Janz et al. [116] either at $1096 \mathrm{~K}$ or extrapolated to $1096 \mathrm{~K}$.

\subsubsection{Testing Convergence for FPMD Simulations}

\subsubsection{Creating converged liquid structures}

Equilibrating a liquid molten salt from a solid, crystalline structure was not feasible within timescales of up to 18 ps at 1000K with FPMD (Figure 4.2(a)). Radial distribution functions (RDFs) confirm that FPMD simulations starting from a solid structure still retain long-range order (Figure 4.2(a)). While more disordered starting conditions and higher temperatures could certainly accelerate the convergence, we chose to simply initiate the FPMD results with IPMD data. IPMD simulations were used to equilibrate all liquid structures by the approach discussed in the Computational methods section and showed converged stable liquid RDFs (Figure 4.2(b)) after equilibration following the approach described in the Computational methods section. The resulting equilibrated IPMD liquid was used as the starting structure for the FPMD calculations and stable liquid RDFs were obtained with FPMD (Figure 4.2(c)). RDFs show the same liquid structure in IPMD and FPMD and longer simulations in the FPMD do not significantly alter the RDFs (although they do get smoother). The slight variations in $\mathrm{Li}-\mathrm{Li}$ interactions between IPMD and FPMD are probably due to the differences between interatomic potentials and the full density functional theory of FPMD. 

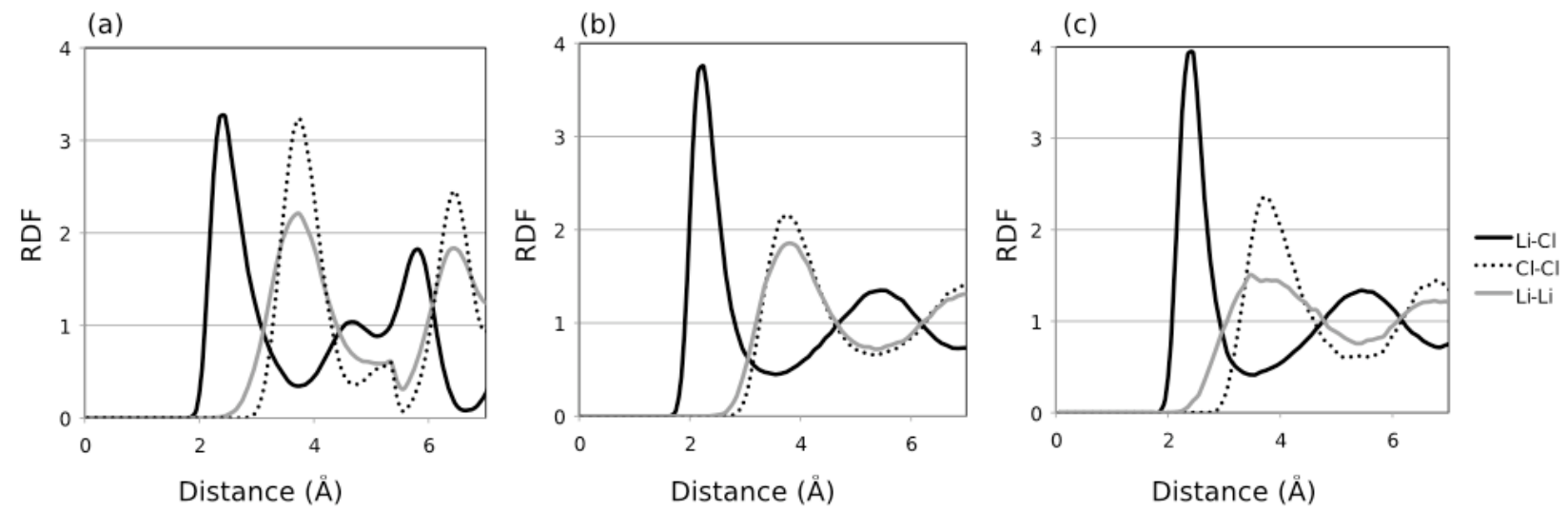

Figure 4.2. Structural parameters for the 64-atom $\mathrm{LiCl}$ structure. (a) FPMD calculations started from solid, crystalline structure and run at $1000 \mathrm{~K}$ for $18 \mathrm{ps}$. Long-range order is not lost, suggesting the material is not molten. (b) Classical IPMD simulations using the LAMMPS code run for 100 ps. (c) FPMD calculations starting from liquid structure equilibrated in LAMMPS from part (b) run for 6 ps.

\subsubsection{Testing convergence with simulation time}

One concern of running FPMD for only 5-10 ps is that the time scales are not long enough to yield converged results for temperature, volumes, energies, pressures, and diffusivities. To test the convergence of these properties with simulation time, IPMD NVT simulations were started from the same converged IPMD simulation that would be used to start a VASP simulation (represented by $\mathrm{t}=0$ in Figure 4.3). Simulations with 64 atoms at $1096 \mathrm{~K}$ were run with IPMD for 6, 12, 20, 50, 100 and 200 ps (Figure 4.3). We assess convergence by considering if the predicted value and errors at some smaller time are consistent with the value at the longest time, which we take to be essentially the exact prediction. This approach does not mean the shorter simulation has a small error bar, only that the values and errors are estimated robustly enough that they are consistent with the exact prediction.

Starting from an equilibrated liquid, a simulation time of 6 ps is long enough to obtain temperatures and energy values that are within the standard deviation of the mean of the values at 200 ps. The pressure at $6 \mathrm{ps}$ is not within one standard deviation of the pressure at $200 \mathrm{ps}$; the pressure at $6 \mathrm{ps}$ is slightly higher than the pressure at 200 ps by $0.07 \pm 0.05 \mathrm{GPa}$. To obtain diffusion coefficients that are within a standard deviation of the diffusion coefficients at $50 \mathrm{ps}$, a simulation time of at least 12 ps is needed, especially for $\mathrm{Cl}$ (Figure 4.3(d)). The standard deviation of the diffusion coefficient is still $\sim 10 \%$ or more of its value of the self-diffusion coefficient, even after 50 ps. 
Figure 4.3. Convergence of (a) temperature (b) pressure (c) total energy and (d) self-diffusion coefficients with simulation time. Simulations were run for 6, 12, 20, 50, 100, and 200 ps. All simulations were run as NVT with IPMD (using LAMMPS) for LiCl with 64 atoms at $1096 \mathrm{~K}$ with a timestep of $2 \mathrm{fs}$. All simulations started from the same converged IPMD simulation that would be used to start a VASP (FPMD) simulation. Desired simulation times for VASP are 5-10 ps. Errors were calculated from the autocovariance function. Averaging started after 0.5 ps so as to use only the equilibrated region.

\subsubsection{Testing convergence with unit cell size}

Additional convergence testing was run in IPMD to test if unit cells of 50-200 atoms are large enough to give the same results as up to 1000-atom unit cells during the reasonable simulation time (100 ps) (Figure 4.4). Simulations for 64, 100, 216, 400, and 1000 atoms were equilibrated in IPMD using NVE (crude barostat), NPT ( $\mathrm{P}=0$ ) for 100,000 time steps, and the NVT for 100,000 time steps. Volumes and errors in Figure 4.4(a) were obtained from NPT and pressure (b), energy (c) and temperature (d) were obtained from NVT simulations. Properties estimated using a unit cell size of 64 atoms and 100 atoms have larger error bars compared to the values at a bigger cell. Volume and pressure values estimated from a cell size of 64 atoms deviate $\sim 1 \%$ and $0.04 \mathrm{GPa}$, respectively, from the 1000-atom values. Estimated energies using a smaller cell also deviate $\sim 5 \mathrm{meV} /$ atom from the 1000-atom value. A unit cell of at least 216 atoms is therefore needed for accurate energies on this scale. Temperature changes little with unit cell size however, the standard deviation decreases as the unit cell size increases. As can be seen in Figure 4.4, a unit cell size of at least 216 atoms is preferable to obtain results that are converged with respect to larger unit cells. However, given a 
speed-up of an almost 8 times for a 64-atom unit cell over a 216-atom unit cell, 64-atom unit cells can be used for quicker testing without sacrificing significant accuracy.

(a)

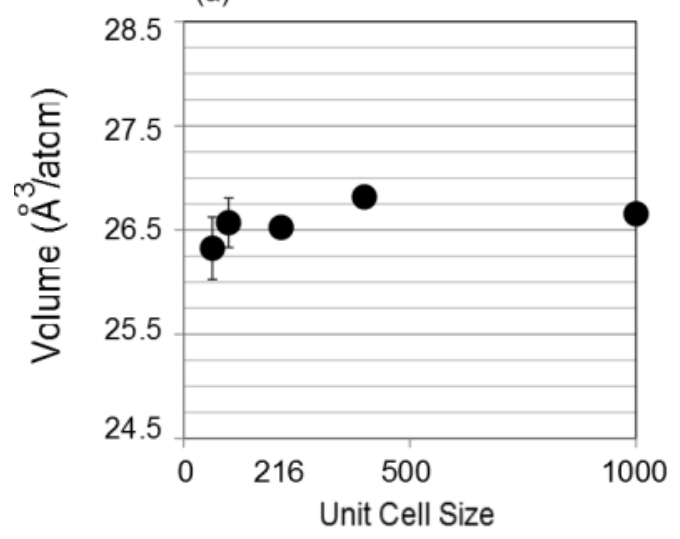

(c)

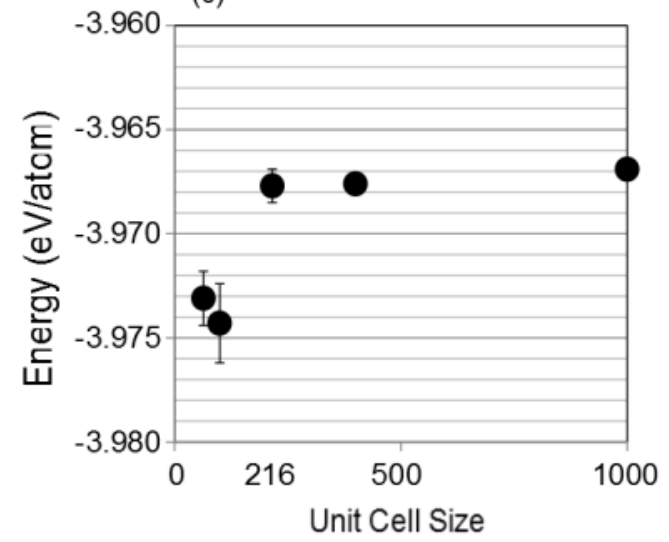

(b)

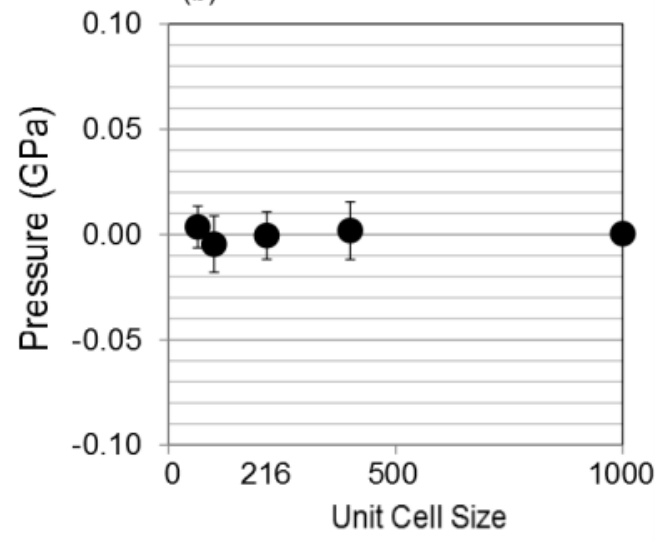

(d)

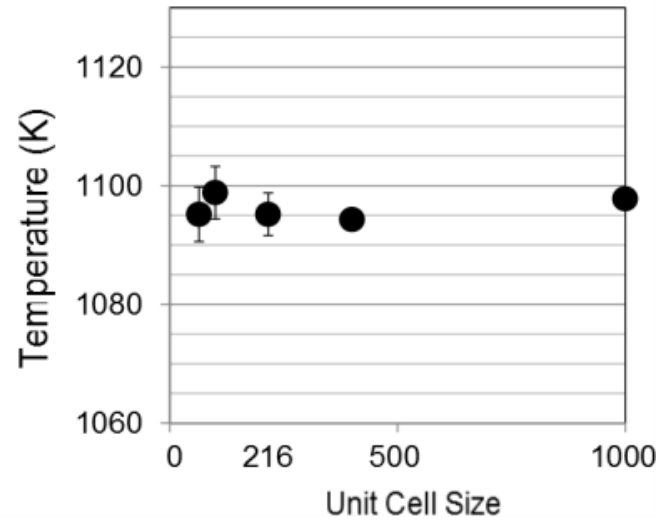

Figure 4.4. Convergence of (a) volume (b) pressure (c) energy and (d) temperature with unit cell size in LiCl. Simulations were run with IPMD with the method outlined in the Computational methods section (NVE, crude barostat, NPT (P=0) for 100,000 steps, NVT for 100,000 steps). Volumes and errors in (a) come from NPT simulation analyzed over last $90 \%$ of data set. (b, c, d) results are from NVT simulations where the last $90 \%$ of simulation was analyzed. Errors are from analytical method.

\subsubsection{Results for Pure $\mathrm{LiCl}-\mathrm{KCl}$ Salts}

\subsubsection{Equilibrium volumes, thermal expansion, bulk modulus}

In order to determine equilibrium volume $\left(\mathrm{V}_{0}\right)$ from NVT simulations, multiple volumes were run for $\mathrm{LiCl}, \mathrm{KCl}$ and the eutectic composition at multiple temperatures (Figure 4.5). Experimental data are plotted as open markers for reference. The resulting pressure-volume data and resulting errors were fit to the Murnaghan equation of state to obtain $\mathrm{V}_{0}$ and the equation of state properties (Table 4.1). In LiCl-KCl eutectic, the equilibrium volumes agree well with available experimental data for all temperatures, but equilibrium volumes are slightly underestimated or overestimated for $\mathrm{LiCl}$ and $\mathrm{KCl}$ (Figure 4.5, Figure 4.6, and Table 4.1).

Coefficients of thermal expansion [96, 117]: 
$\alpha_{V}=\frac{1}{V}\left(\frac{\partial V}{\partial T}\right)_{P}$

at a specific volume were calculated for each composition and temperature from the slope of the volume vs. temperature plot (Figure 4.6 and Table 4.2). Volumes as a function of temperature from FPMD are shown in comparison to experimental data (Figure 4.6). Experimental data in the LiCl$\mathrm{KCl}$ eutectic composition has the most variability. Calculated volumes for the eutectic composition fall within the error bars of the experimental data (dashed line). Volumes for $\mathrm{LiCl}$ are underestimated by about $4-5 \%\left(\sim 1 \AA^{3} /\right.$ atom $)$ and volumes for $\mathrm{KCl}$ are overestimated by about 2-3\% ( $\sim 1 \AA^{3} /$ atom) by the calculations compared to the experiments.

Bulk modulus (B) decreases with increasing temperature (Figure 4.7), consistent with Garai and Laugier [118]. $\mathrm{LiCl}$ has highest $B ; \mathrm{KCl}$ has the lowest $B$. The eutectic $B$ is between $\mathrm{LiCl}$ and $\mathrm{KCl}$, as would be expected. The bulk modulus for these liquids is quite low ( $<8 \mathrm{GPa}$ ) compared to solid $\mathrm{LiCl}$ ( $B \sim 30 \mathrm{GPa}$ at room temperature) [119] and solid $\mathrm{KCl}$ ( $B \sim 17 \mathrm{GPa}$ at room temperature) [118]. $B$ ', the change in $B$ with pressure, was difficult to constrain with values ranging from 4-12 and uncertainties of up to about \pm 2 .

Table 4.1. Equilibrium volume $\left(\mathrm{V}_{0}\right)$ from FPMD with 216 atoms. Simulations were run at multiple volumes for the NVT ensemble. $\mathrm{V}$, $\mathrm{P}$ were fit to a Murnaghan equation of state to obtain $\mathrm{V}_{0}$ (Experimental $\mathrm{V}_{0}$ : Janz et al. (1979) [116] (extrapolated for some temperatures))

\begin{tabular}{|c|c|c|c|c|c|c|}
\hline & $\begin{array}{c}\% \\
\mathrm{LiCl}\end{array}$ & $\begin{array}{c}\mathrm{T} \\
(\mathrm{K})\end{array}$ & $\begin{array}{c}\mathrm{V}_{0} \\
\text { Experiment } \\
\left(\AA^{3} / \mathrm{atom}\right)\end{array}$ & $\mathrm{V}_{0}\left(\AA^{3} /\right.$ atom $)$ & B (GPa) & $\mathrm{B}^{\prime}$ \\
\hline \multirow{3}{*}{$\mathrm{LiCl}$} & \multirow{3}{*}{100} & 1096 & 24.93 & $23.73 \pm 0.005$ & $4.95 \pm 0.02$ & $9.30 \pm 0.16$ \\
\hline & & 1000 & 24.25 & $23.03 \pm 0.001$ & $5.41 \pm 0.19$ & $7.42 \pm 1.35$ \\
\hline & & 900 & 23.54 & $22.39 \pm 0.052$ & $7.01 \pm 0.28$ & $12.32 \pm 1.70$ \\
\hline \multirow{2}{*}{$\mathrm{KCl}$} & \multirow{2}{*}{0} & 1200 & 43.10 & \multicolumn{3}{|c|}{ Could not fit with EOS* } \\
\hline & & 1096 & 41.42 & $42.52 \pm 0.167$ & $2.79 \pm 0.18$ & $6.90 \pm 1.91$ \\
\hline \multirow{3}{*}{$\begin{array}{c}\text { LiCl- } \\
\text { KCl } \\
\text { eutectic }\end{array}$} & \multirow{3}{*}{58.3} & 1096 & 31.32 & $31.72 \pm 0.064$ & $3.64 \pm 0.07$ & $5.88 \pm 0.90$ \\
\hline & & 1000 & 30.54 & $30.89 \pm 0.037$ & $4.67 \pm 0.11$ & $3.69 \pm 0.51$ \\
\hline & & 800 & 28.67 & \multicolumn{3}{|c|}{ Could not fit with EOS* } \\
\hline
\end{tabular}

* Due to the lack of $\mathrm{P}(\mathrm{V})$ data, EOS fit could not be made

Table 4.2. Coefficient of thermal expansion, $\alpha_{V}$ at $1096 \mathrm{~K}$ from FPMD with 216 atoms.

\begin{tabular}{|c|c|c|c|c|}
\hline & $\% \mathrm{LiCl}$ & Temperature (K) & $\alpha_{\mathrm{V}}$ FPMD $(1 / \mathrm{K}) \times 10^{-4}$ & $\alpha_{\mathrm{V}}$ Experiment $(1 / \mathrm{K}) \times 10^{-4}$ \\
\hline $\mathrm{LiCl}$ & 100 & 1096 & 2.87 & $2.94 \pm 0.00$ \\
\hline $\mathrm{KCl}$ & 0 & 1096 & - & $3.94 \pm 0.02$ \\
\hline $\mathrm{LiCl}-\mathrm{KCl}$ & 58.3 & 1096 & 2.73 & $2.93 \pm 0.05$ \\
\hline
\end{tabular}


(a) $\mathrm{LiCl}$

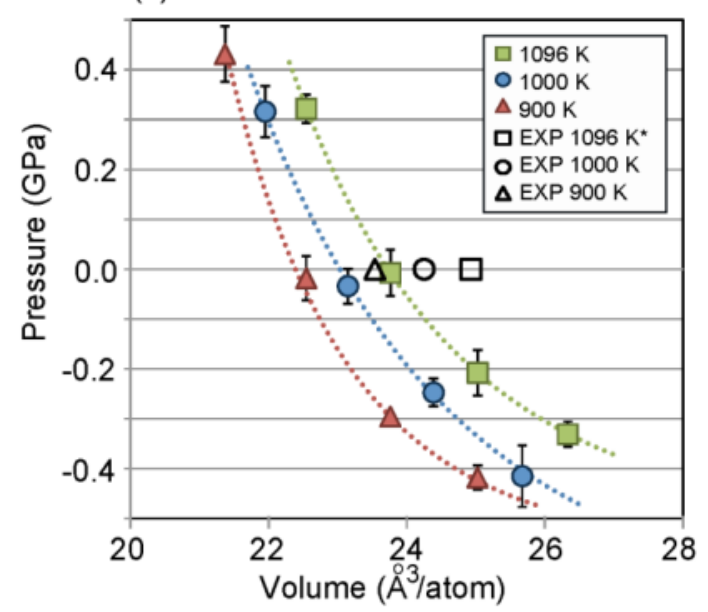

(b) $\mathrm{KCl}$

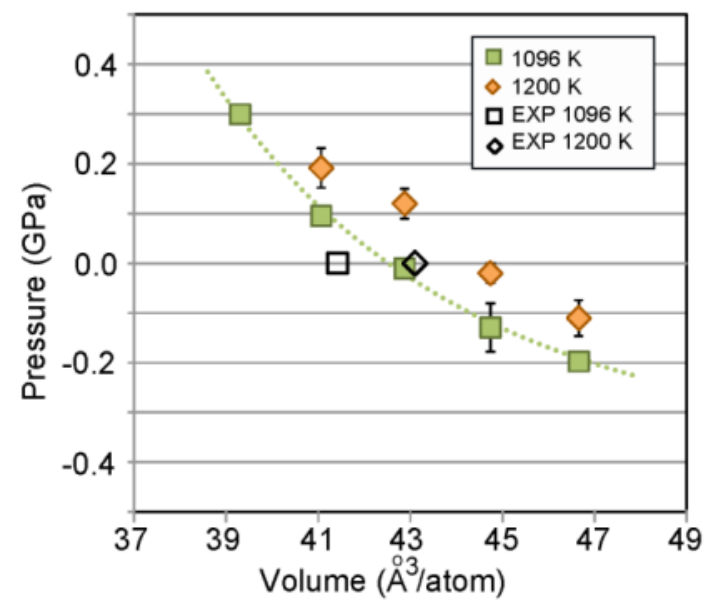

(c) $\mathrm{LiCl}-\mathrm{KCl}$ eutectic

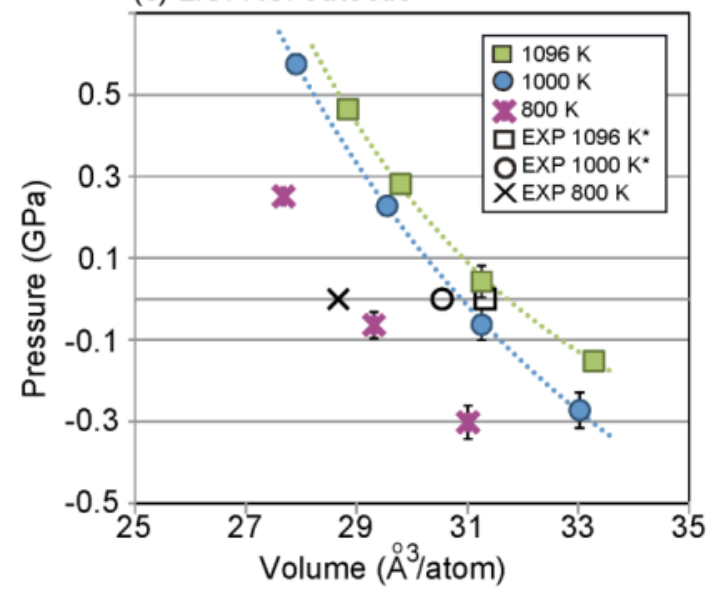

Figure 4.5. Pressure-volume curves from VASP FPMD simulations with the NVT ensemble for of (a) LiCl (b) $\mathrm{KCl}$ and (c) $\mathrm{LiCl}-\mathrm{KCl}$ with the eutectic composition. Experimental volumes are from (*extrapolated from) Janz et al. [116]. 

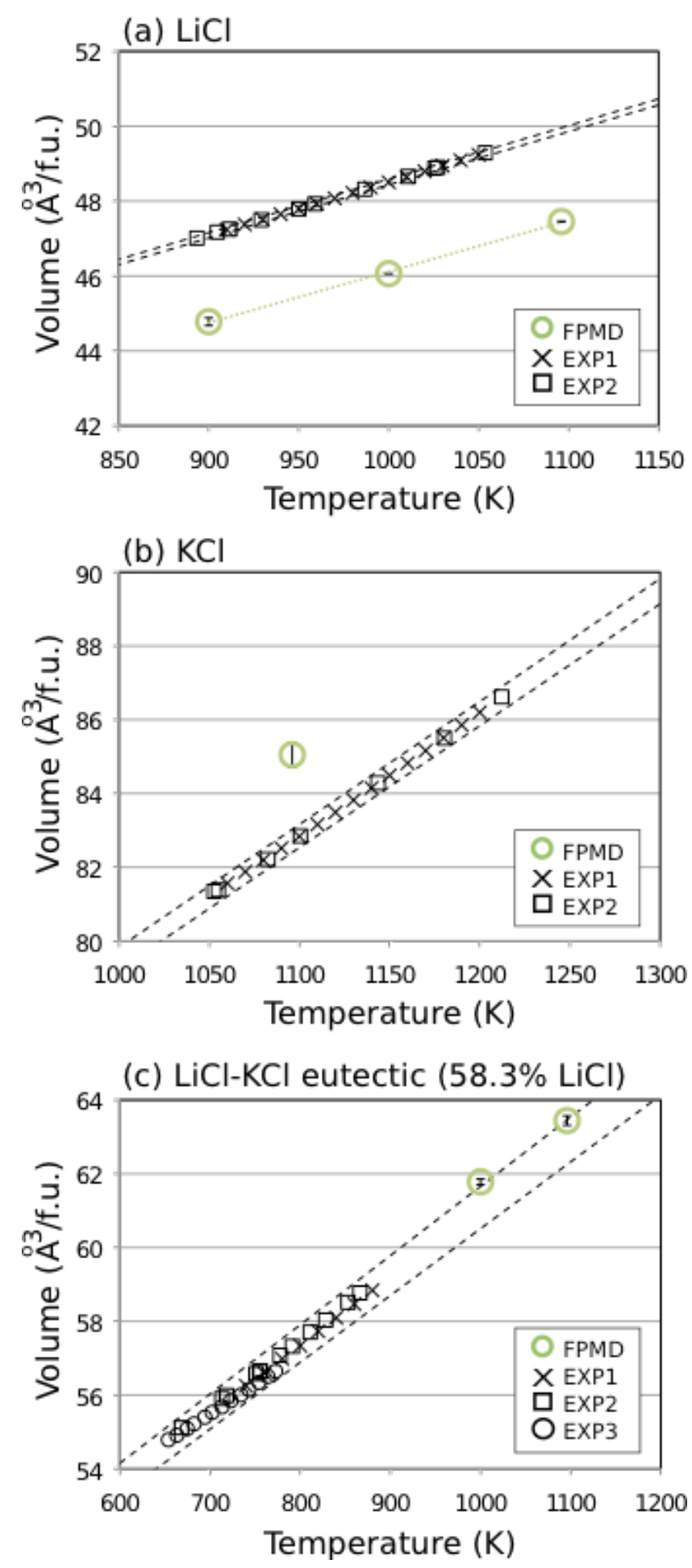

Figure 4.6. Volume vs. temperature for (a) $\mathrm{LiCl}$ (b) $\mathrm{KCl}$ and (c) $\mathrm{LiCl}-\mathrm{KCl}$ with the eutectic composition at multiple temperatures. LiCl-KCl volumes are plotted per Cl atom. EXP1 = Janz et al. (1979) [116]. EXP2 = Van Artsdalen and Yaffe (1955) [120], EXP3 = Ito et al. (2001) [121]. Dashed lines indicate the experimental error between the experimental datasets. 


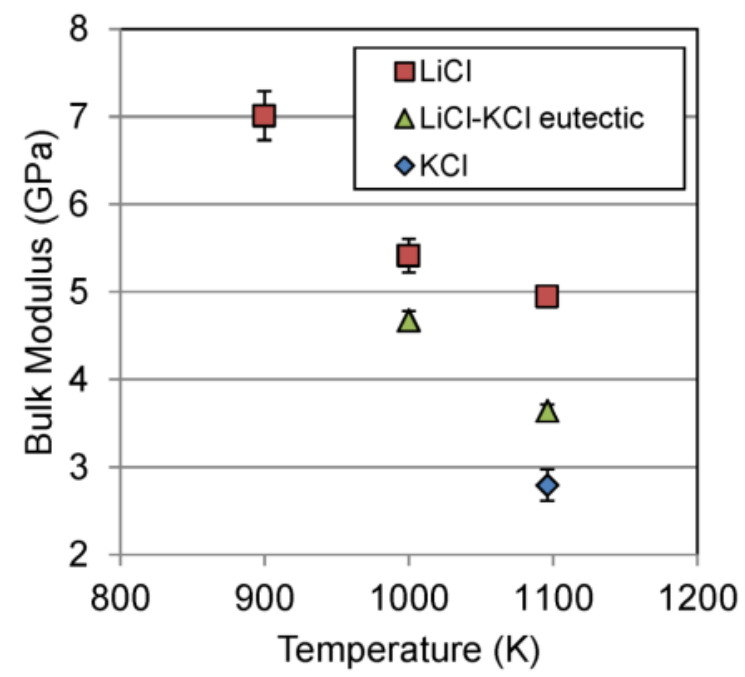

Figure 4.7. Bulk modulus vs. temperature for the LiCl-KCl system.

\subsubsection{Self-diffusion coefficients}

The self-diffusion coefficients were calculated for $\mathrm{Li}, \mathrm{Cl}$ and $\mathrm{K} \mathrm{in} \mathrm{LiCl}, \mathrm{KCl}$ and $\mathrm{LiCl}-\mathrm{KCl}$ with the eutectic composition (Figure 4.8, Figure 4.9 and Table 4.3). Experimental and IPMD computational data exists in the literature for comparison in both $\mathrm{LiCl}[88,116,122,123]$ and $\mathrm{KCl}[88,89,98$, 123, 124], but only IPMD computational data exists for self-diffusion in eutectic LiCl-KCl [88, 89].

Thre are a range of values in the experimental self-diffusion coefficients, with different experimental studies showing up to one order of magnitude difference (Morgan and Janz in Figure 4.8 (c) and (d)). For Li diffusivity in LiCl, the FPMD diffusion coefficients fall between the experimental diffusivity and IPMD (literature) results. Cl diffusivity in LiCl calculated from FPMD is slightly lower than the experimental values by Janz et al. [116] and previous IPMD calculations, and show a somewhat larger slope vs. temperature with temperature than data with the widest temperature range [122]. $\mathrm{K}$ and $\mathrm{Cl}$ diffusion in $\mathrm{KCl}$ from FPMD calculations are near experimental and previous IPMD results. The experimental data from Janz et al. [116] in KCl is significantly lower than any other study.

FPMD self-diffusion coefficients for $\mathrm{Li}, \mathrm{Cl}$ and $\mathrm{K}$ in eutectic $\mathrm{LiCl}-\mathrm{KCl}$ (Figure 4.9) agree well with previous IPMD simulations [88, 89], although trends with temperature for Li are slightly steeper than the IPMD results. No error bars were reported in the IPMD simulations. 
Table 4.3. Self-diffusion coefficients for $\mathrm{Li}, \mathrm{Cl}$ and $\mathrm{K}$ in $\mathrm{LiCl}, \mathrm{KCl}$ and eutectic $\mathrm{LiCl}-\mathrm{KCl}$. Mean squared displacement (MSD) were calculated at the volume closest to equilibrium volume from the set of FPMD simulations for the EOS analysis. Each simulation was performed with the NVT ensemble and 216 atoms for 6 ps. MSD analysis started after the first 200 time steps (for equilibration). The remaining time steps were divided into 4 equal blocks. The MSD vs. time curve was fit for all but the initial 25 steps of each block in order to avoid the quadratic region.

\begin{tabular}{|c|c|c|c|c|c|c|}
\hline & $\begin{array}{c}\% \\
\mathrm{LiCl}\end{array}$ & $\begin{array}{l}\text { Volume } \\
\AA^{3} / \text { atom }\end{array}$ & Temp (K) & $\begin{array}{l}\text { Diffusion Li } \\
\mathrm{cm}^{2} / \mathrm{s} \times 10^{-5}\end{array}$ & $\begin{array}{l}\text { Diffusion K } \\
\mathrm{cm}^{2} / \mathrm{s} \times 10^{-5}\end{array}$ & $\begin{array}{l}\text { Diffusion Cl } \\
\mathrm{cm}^{2} / \mathrm{s} \times 10^{-5}\end{array}$ \\
\hline \multirow{3}{*}{$\mathrm{LiCl}$} & \multirow{3}{*}{100} & 23.76 & 1096 & $14.04 \pm 0.66$ & - & $7.18 \pm 0.58$ \\
\hline & & 23.15 & 1000 & $11.58 \pm 0.39$ & - & $5.26 \pm 0.27$ \\
\hline & & 22.55 & 900 & $8.10 \pm 0.30$ & - & $3.77 \pm 0.20$ \\
\hline \multirow{2}{*}{$\mathrm{KCl}$} & \multirow[t]{2}{*}{0} & 44.74 & 1200 & - & $12.11 \pm 0.39$ & $11.38 \pm 0.74$ \\
\hline & & 42.88 & 1096 & - & $8.39 \pm 0.36$ & $8.6 \pm 0.25$ \\
\hline \multirow{3}{*}{$\begin{array}{c}\text { LiCl- } \\
\text { KCl } \\
\text { eutectic }\end{array}$} & \multirow{3}{*}{59.4} & 31.26 & 1096 & $10.23 \pm 0.84$ & $9.78 \pm 0.63$ & $7.99 \pm 0.68$ \\
\hline & & 31.26 & 1000 & $8.83 \pm 0.40$ & $6.93 \pm 0.24$ & $6.29 \pm 0.16$ \\
\hline & & 29.31 & 800 & $4.37 \pm 0.27$ & $4.89 \pm 0.50$ & $3.70 \pm 0.08$ \\
\hline
\end{tabular}


(a) $\mathrm{Li}$ in $\mathrm{LiCl}$

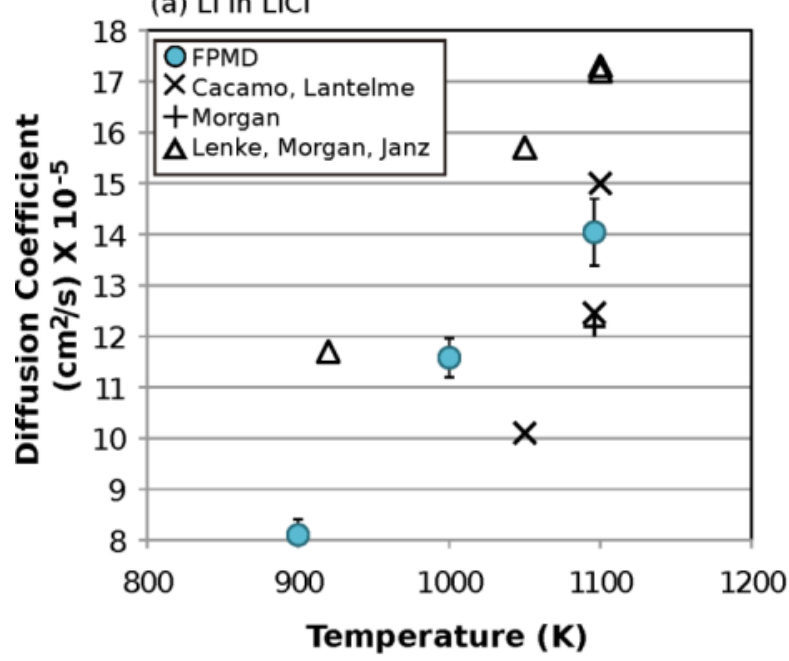

(c) $\mathrm{K}$ in $\mathrm{KCl}$

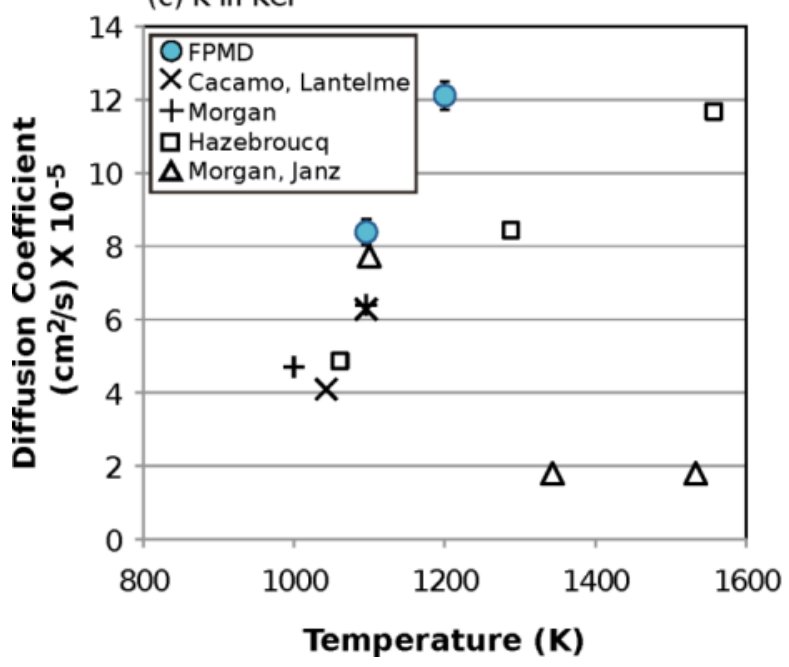

(b) $\mathrm{Cl}$ in $\mathrm{LiCl}$

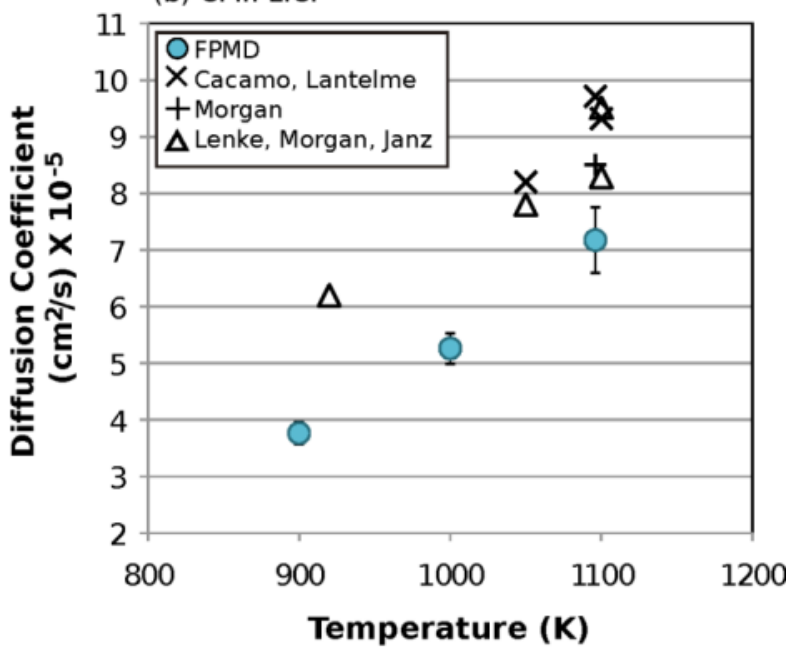

(d) $\mathrm{Cl}$ in $\mathrm{KCl}$

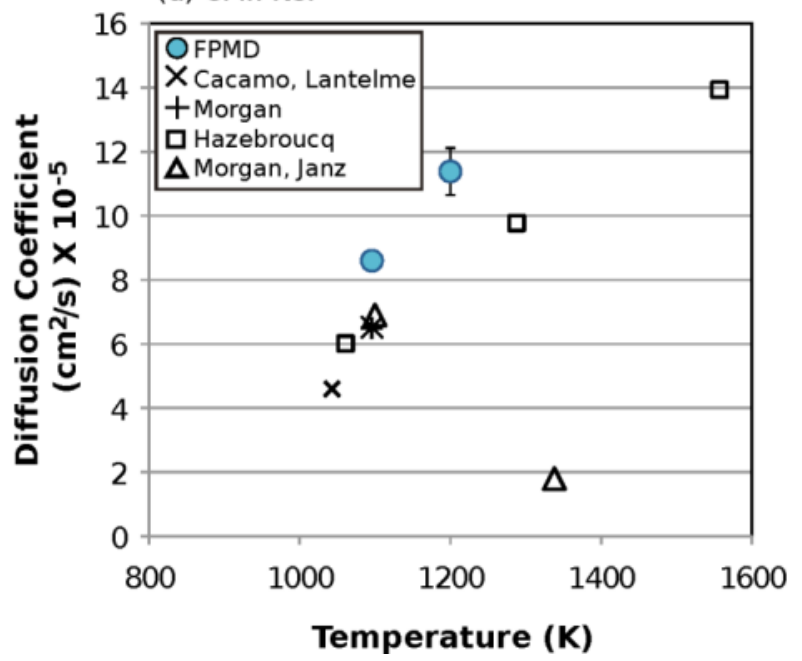

Figure 4.8. Self-diffusion coefficients from FPMD simulations for (a) $\mathrm{Li}$ in $\mathrm{LiCl}$ (b) $\mathrm{Cl}$ in $\mathrm{LiCl}$ (c) $\mathrm{K}$ in $\mathrm{KCl}$ and (d) $\mathrm{Cl}$ in $\mathrm{KCl}$. Experimental values for $\mathrm{LiCl}$ are from Lenke et al. [122] and Janz et al. [116] and references within Lantelme and Turq [88] and Morgan and Madden [123]. Computational IPMD values for $\mathrm{LiCl}$ are taken from Lantelme and Turq [88], Caccamo and Dixon [89], and Morgan and Madden [123] and references within. Experimental values for $\mathrm{KCl}$ are from Morgan and Madden [123] and Janz [124] and references within. Computational IPMD values for $\mathrm{KCl}$ are from Lantelme and Turq [88], Caccamo and Dixon [89], Hazebroucq et al. [98], and Morgan and Madden [123]. All simulations were run with FPMD in VASP at the volume closest to equilibrium (LiCl: $23.76 \AA^{3}$ /atom at $1096 \mathrm{~K}, 23.15 \AA^{3}$ /atom at $1000 \mathrm{~K}, 22.55$ $\AA^{3}$ /atom at $900 \mathrm{~K} ; \mathrm{KCl}: 44.74 \AA^{3}$ /atom at $1200 \mathrm{~K}, 42.88 \AA^{3}$ /atom at $1096 \mathrm{~K}$ ) with the NVT ensemble and 216 atoms for $6 \mathrm{ps}^{1}$. Analysis started after the first 200 time steps (for equilibration). The remaining time steps were divided into 4 equal blocks. The MSD vs. time curve was fit for all but the initial 25 steps of each block in order to avoid the quadratic region.

\footnotetext{
${ }^{1}$ Simulations in $\mathrm{KCl}$ were run for $5.4 \mathrm{ps}$ (2689 time steps) at $1200 \mathrm{~K}$ and $7.3 \mathrm{ps}$ (3641 time steps) at 1096K.
} 

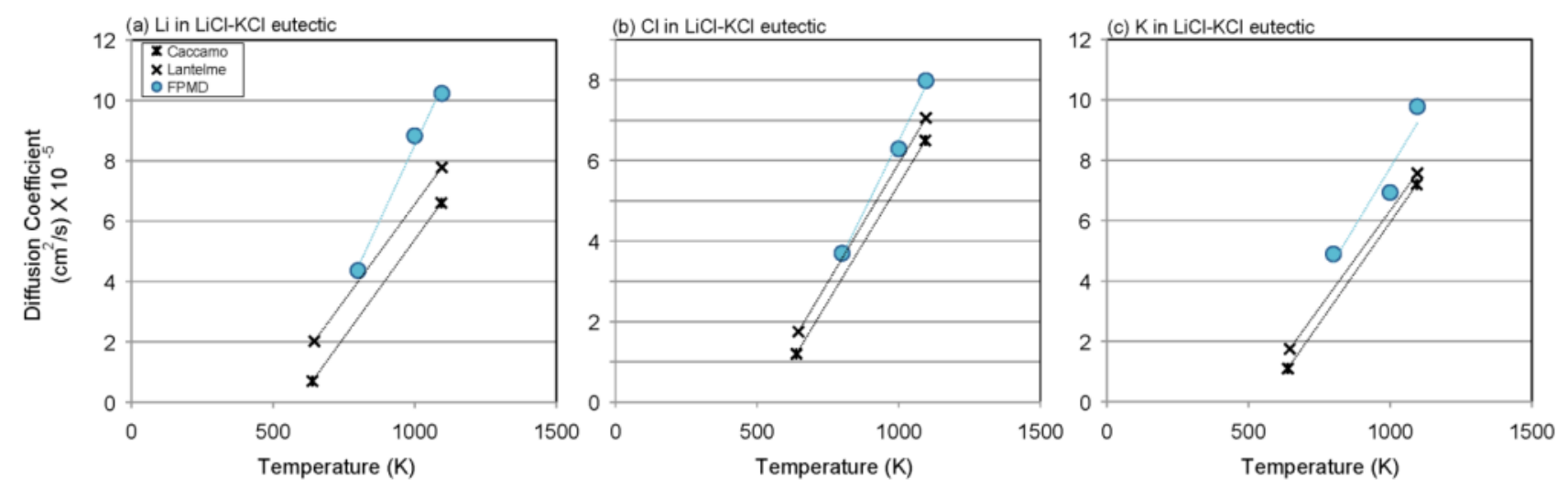

Figure 4.9. Self-diffusion coefficients from FPMD simulations for (a) $\mathrm{Li}$, (b) $\mathrm{Cl}$ and (c) $\mathrm{K}$ in eutectic LiCl$\mathrm{KCl}$. All simulations were run with FPMD in VASP at the volume closest to equilibrium $\left(31.26 \AA^{3} /\right.$ atom at $1096 \mathrm{~K}, 31.26 \AA^{3}$ /atom at $1000 \mathrm{~K}$, and $29.31 \AA^{3}$ /atom at $800 \mathrm{~K}$ ) with the NVT ensemble and 216 atoms for 6 $\mathrm{ps}^{2}$. Analysis started after the first 200 time steps (for equilibration). The remaining time steps were divided into 4 equal blocks. The MSD vs. time curve was fit for all but the initial 25 steps of each block in order to avoid the quadratic region. Results are plotted with IPMD simulations from the literature: Lantelme and Turq [88] and Caccamo and Dixon [89] .

\subsubsection{Gibbs free energy of mixing}

The Gibbs free energy of mixing $\left(\Delta G_{m i x}\right)$ at a given temperature can be estimated from an ideal solution model as [117]:

$$
\begin{aligned}
& \Delta G_{m \bar{x}}=E_{x L i C l(1-x) K C l}-x E_{L E C l}-(1-x) E_{K C l}-T S_{m i x} \\
& =E_{x L I C L I(1-x) K C l}-x E_{L I C l}-(1-x) E_{K C l}+R T[x \ln x+(1-x) \ln (1-x)]
\end{aligned}
$$

where $x$ is the concentration of $\mathrm{LiCl}$. Total energy values from FPMD with 64-atom unit cell (19 Li, $32 \mathrm{Cl}, 13 \mathrm{~K}$; $\mathrm{x}=0.594$ ) were used to estimate the free energy of mixing at the eutectic composition at $1096 \mathrm{~K} . \Delta G_{\text {mix }}$ from FPMD is in good agreement with the experimental fit (Figure 4.10). The FPMD data is only lower than experimental fit by $\sim 0.9 \mathrm{~kJ} / \mathrm{mol}(<10 \mathrm{meV} / \mathrm{Cl}$ atom), which is within our error tolerance for the FPMD simulations. This shows that the excess entropy of mixing for this molten salt system is close to zero at high temperature and the free energy of mixing can be estimated from the ideal solution model.

\footnotetext{
${ }^{2}$ Simulations at $800 \mathrm{~K}$ were only run for 5.5 ps (2744 time steps).
} 
perimental fit

$x$ of $\mathrm{LiCl}$ in $\mathrm{LiCl}-\mathrm{KCl}$

Figure 4.10. Gibbs free energy at $1096 \mathrm{~K}$. The fit to experimental data (dashed line) comes from the FTsalt FACT salt database (FactSage) [125].

\subsubsection{Discussion and Conclusions}

FPMD calculations were used to calculate the equilibrium volume at multiple temperatures, thermal expansion, bulk modulus, and self-diffusion coefficients for $\mathrm{LiCl}, \mathrm{KCl}$ and eutectic $\mathrm{LiCl}-\mathrm{KCl}$ molten salts as well as the Gibbs free energy of mixing. Convergence testing proved unit cells of 216 atoms and simulation times of 6-12 ps are sufficient for adequately converged results that overall agree well with experimental data and classical IPMD simulations. Convergence errors on FPMD data with these settings are small enough to provide useful information on salt properties while still being practical.

The results show that FPMD calculations are able to reliably calculate the equilibrium volume, using NVT simulations, with reasonable agreement with experiments (agreement to within $5 \%$ for $\mathrm{LiCl}$, agreement to within $3 \%$ for $\mathrm{KCl}$ and $1 \% \mathrm{LiCl}-\mathrm{KCl}$ ). Statistical convergence errors on FPMD volumes are typically less than $0.1 \AA^{3}$ /atom (less than $0.5 \%$ ). Previous simulations with IPMD and DFT-TB fixed the volume to experimental values [88, 89, 98, 99]. The Tosi-Fumi potentials used in IPMD simulations [88, 89] overestimate the experimental volume by 6-7 \% (Figure 4.1(a)) corresponding to a pressure of $0.31 \mathrm{GPa}$ in the simulations [88]. The FPMD simulations, which calculate the equilibrium volume independent of experiments, can be extended to predict volumes and densities in systems where little or no experimental data exists.

Calculations can provide guidance when experimental measurements have a wide range of value, as in the self-diffusion coefficients shown in Figure 4.8. Our FPMD results with 216-atoms have good agreement with most of the experimental measurements $[88,116,122,123]$ and the IPMD calculations $[88,89,98,123]$. Although the results are not provided, even with just a 64-atom unit cell and simulations times of only 12 ps, FPMD can calculate diffusion coefficients with errors of less than $10 \%$.

FPMD simulations are a useful predictive tool for properties like bulk modulus, where no experimental data exists on the LiCl-KCl system. Even more complex thermodynamics, such as the Gibbs free energy of mixing, can be estimated with a simple ideal solution model, which makes aspects of phase stability accessible with FPMD. 
With the exception of finding equilibrium volumes, the FPMD simulations shown here do not significantly improve upon IPMD simulations. Given the significant speedup of IPMD simulations relative to FPMD, IPMD simulations are most efficient for systems where quality potentials can be developed. The real benefit of FPMD simulations is for use in systems where accurate potential fitting proves problematic or there is interest in electronic properties, like redox chemistry or magnetic moment, that are difficult to simulate without full electronic structure methods. The validation shown in this work of FPMD simulations on LiCl-KCl system provides confidence in applying the same techniques to additional systems, including impurities in the LiCl-KCl system or entirely different salts. 


\subsection{FPMD Calculations for Sr/La/U Solutes}

The first principles molecular dynamics (FPMD) modeling of Sr, La and U solutes in the LiCl-KCl eutectic molten salt at $773 \mathrm{~K}\left(500^{\circ} \mathrm{C}\right)$ has conducted, because the $\mathrm{Sr}$, La and $\mathrm{U}$ represent fission product, lanthanides and actinides, respectively. In order to ionize the solute, we put $\mathrm{SrCl}_{2}, \mathrm{LaCl}_{3}$, $\mathrm{UCl}_{3}$, and $\mathrm{UCl}_{4}$ molecules in the 64 atoms of $\mathrm{LiCl}-\mathrm{KCl}$ molten salt $(19 \mathrm{Li}$ atoms, $13 \mathrm{~K}$ atoms, $32 \mathrm{Cl}$ atoms; $59.4 \% \mathrm{LiCl}$ ). From the FPMD simulations, local structure surrounding solutes is predicted by analyzing the radial distribution function. And self-diffusion a coefficient of solutes in molten $\mathrm{LiCl}-\mathrm{KCl}$ is calculated from the mean-squared displacement (MSD) analysis as performed in case of constituents of the LiCl-KCl. The redox potential for each element, which is important property in partitioning technology of nuclear wastes, was also predicted from the FPMD simulations.

For better understanding of $\mathrm{Sr} / \mathrm{La} / \mathrm{U}$ behaviors in the $\mathrm{LiCl}-\mathrm{KCl}$, the FPMD simulations for additional solutes (Eu, $\mathrm{Nd}$, Ce and $\mathrm{Zr}$ ) were also conducted.

\subsubsection{Local Structure Surrounding Solutes in $\mathrm{LiCl}-\mathrm{KCl}$}

\subsubsection{Local structure analysis for $\mathrm{Sr} / \mathrm{La} / \mathrm{U}$ solutes}

Local structure of Sr, La, U ions in the LiCl-KCl molten salts were studied by analyzing the FPMD simulation trajectory. VMD [126] is used to visualize the trajectory of FPMD simulation. Figure 4.11 shows snapshots of FPMD trajectory of LiCl-KCl salt with the dissolved solutes.

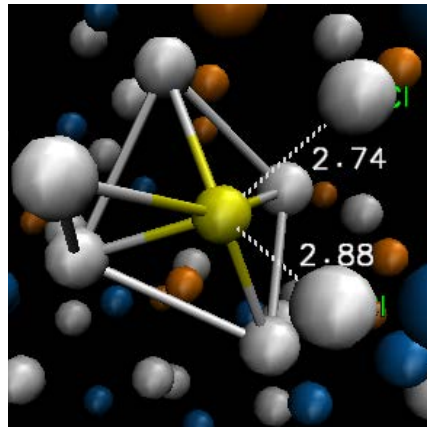

(a)

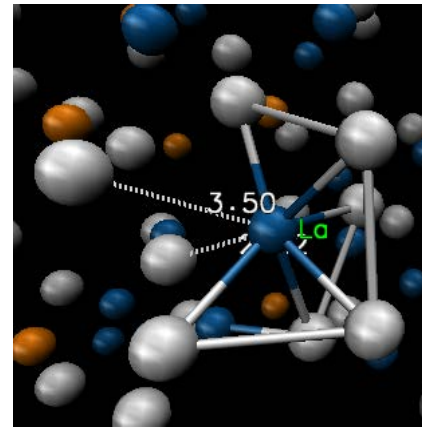

(b)

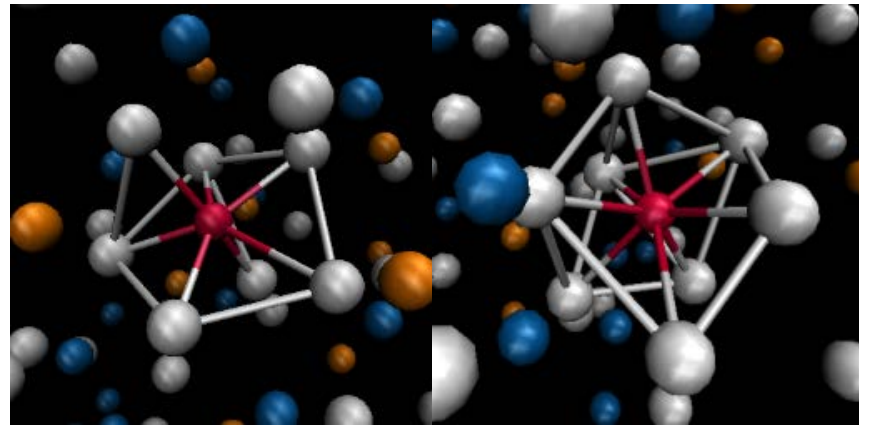

(d)

Figure 4.11. Snapshots of molecular dynamics simulation trajectory, (a) FPMD simulation of the LiCl-KCl with $\mathrm{SrCl}_{2}$ showing bonding between $\mathrm{Sr}$ and $\mathrm{Cl}$ ions; (b) local structure of La from the FPMD simulation of the $\mathrm{LiCl}-\mathrm{KCl}$ with $\mathrm{LaCl}_{3}$; (c) local structure of $\mathrm{U}$ from the FPMD simulation of the $\mathrm{LiCl}-\mathrm{KCl}$ with $\mathrm{UCl}_{3}$; (d) local structure of $\mathrm{U}$ from the FPMD simulation of the $\mathrm{LiCl}-\mathrm{KCl}$ with $\mathrm{UCl}_{4}$

Figure 4.11(a) and (b) were captured from the FPMD trajectory of the $\mathrm{LiCl}-\mathrm{KCl}$ with $\mathrm{SrCl}_{2}$ and $\mathrm{LaCl}_{3}$, respectively. Blue balls are Li ions and orange balls are $\mathrm{K}$ ions. White balls, yellow ball, and red ball are $\mathrm{Cl}$, Sr and $\mathrm{U}$ ions, respectively. Figure 4.11(c) is the snapshot of local structure of $\mathrm{U}^{3+}$ in the $\mathrm{LiCl}-\mathrm{KCl}$. Figure 4.11(d) is showing the local structure of $\mathrm{U}^{4+}$ in the salt.

To quantify the local coordination of solutes with surrounding chlorine ions, RDF (radial distribution function) analysis were conducted. Figure 4.12 shows RDFs of the LiCl-KCl with $\mathrm{SrCl}_{2}$, $\mathrm{LaCl}_{3}, \mathrm{UCl}_{3}$ and $\mathrm{UCl}_{4}$, respectively. When solute has higher oxidation states, it will make more bonds with $\mathrm{Cl}$ ions and the first peak is greater. Coordination number of solute with surrounding $\mathrm{Cl}$ 
ions can be estimated by integrating the radial distribution function (RDF) from zero to the first minimum. Calculated coordination numbers of $\mathrm{Sr}(2+), \mathrm{La}(3+), \mathrm{U}\left(3^{+}\right)$and $\mathrm{U}(4+)$ are about 6.9, 7.4, 7.0 and 7.1, respectively.

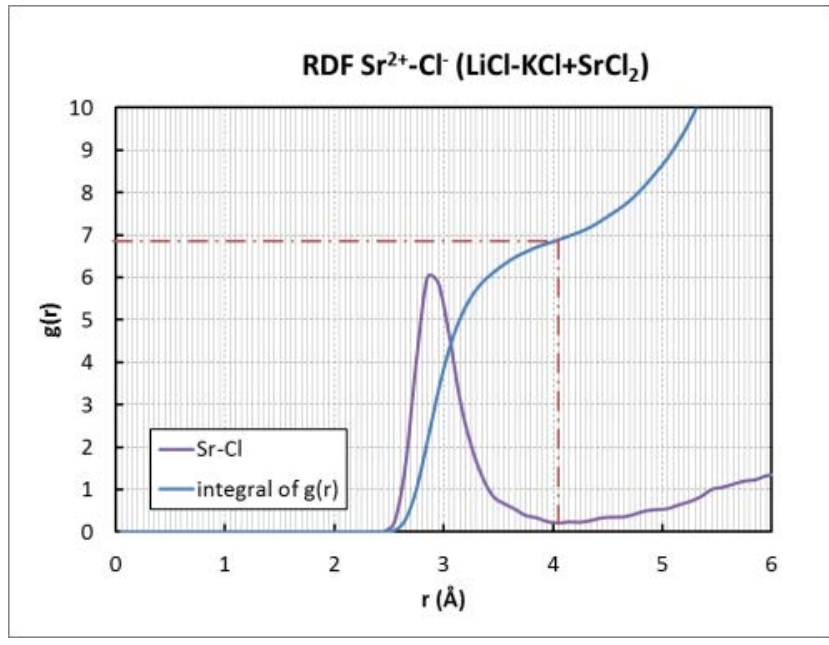

(a)

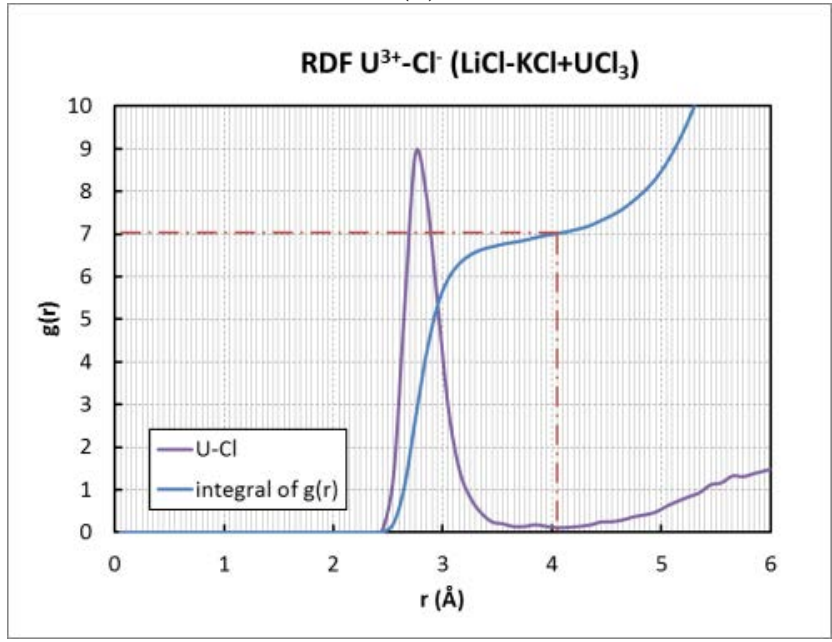

(c)

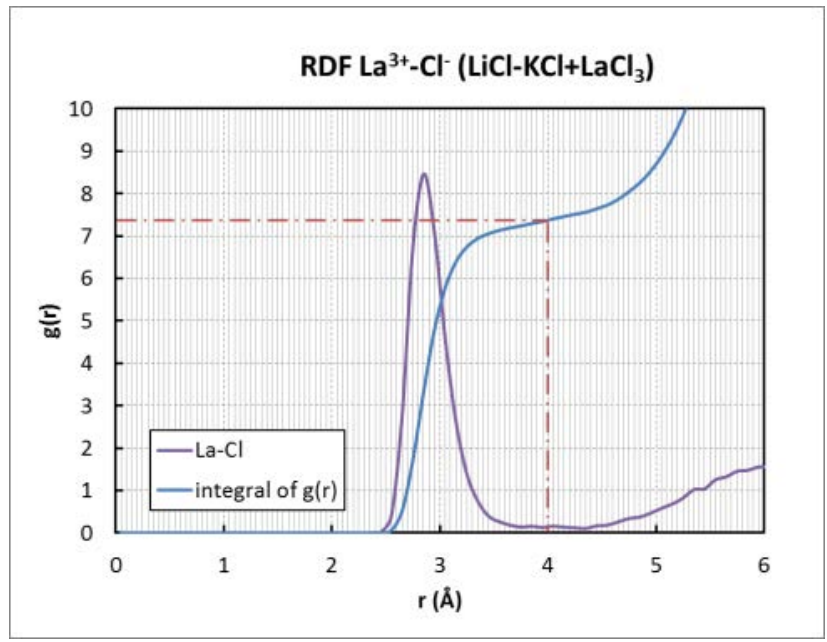

(b)

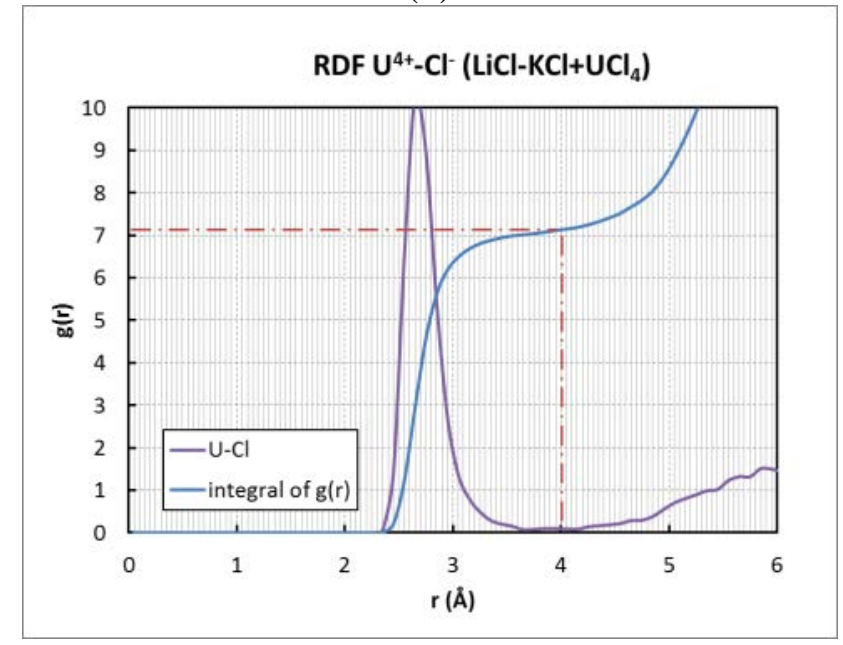

(d)

Figure 4.12. Radial distribution functions (RDF) of the $\mathrm{LiCl}-\mathrm{KCl}$ (64 atoms) with (a) $\mathrm{SrCl}_{2}$; (b) $\mathrm{LaCl}_{3}$; (c) $\mathrm{UCl}_{3}$; (d) $\mathrm{UCl}_{4}$ between each solute and $\mathrm{Cl}$ ions.

\subsubsection{Local structure analysis for other solutes (Eu/Nd/Ce/Zr)}

Local structure of $\mathrm{Eu}, \mathrm{Nd}, \mathrm{Ce}$ and $\mathrm{Zr}$ ions in the $\mathrm{LiCl}-\mathrm{KCl}$ molten salts were also studied by analyzing the FPMD simulation trajectory. Figure 4.13 shows snapshots of FPMD trajectory of $\mathrm{LiCl}-\mathrm{KCl}$ salt with the dissolved solutes.

Figure 4.13(a)-(d) were captured from the FPMD trajectory of the $\mathrm{LiCl}-\mathrm{KCl}$ with $\mathrm{EuCl}_{3}, \mathrm{NdCl}_{3}$, $\mathrm{CeCl}_{3}$ and $\mathrm{ZrCl}_{4}$, respectively. Blue, orange and white balls are $\mathrm{Li}, \mathrm{K}$ and $\mathrm{Cl}$ ions. As each solute dissolved in the salt is cation, it makes bonds with surrounding anions, i.e. $\mathrm{Cl}^{-}$ions. Although bonding behavior of solutes can be observed from the trajectory movies, radial distribution function (RDF) is used to analyze the bonding behavior quantitatively. 


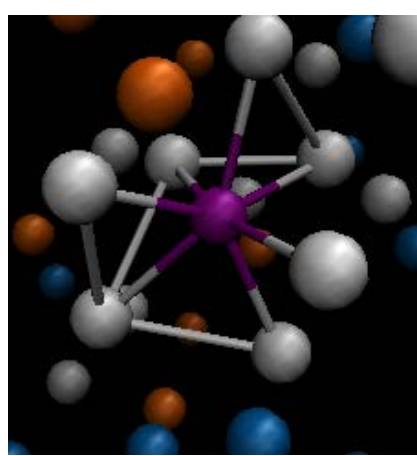

(a)

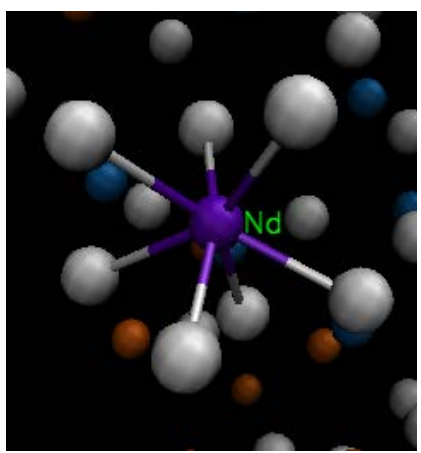

(b)

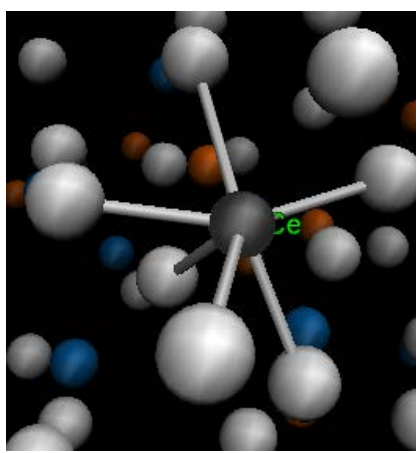

(c)

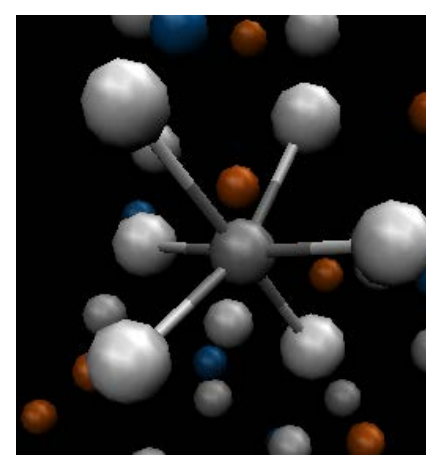

(d)

Figure 4.13. Snapshots of molecular dynamics simulation trajectory, (a) FPMD simulation of the LiCl-KCl with $\mathrm{EuCl}_{3}$ showing bonding between $\mathrm{Eu}$ and $\mathrm{Cl}$ ions; (b) local structure of $\mathrm{Nd}$ from the FPMD simulation of the $\mathrm{LiCl}-\mathrm{KCl}$ with $\mathrm{NdCl}_{3}$; (c) local structure of Ce from the FPMD simulation of the $\mathrm{LiCl}-\mathrm{KCl}$ with $\mathrm{CeCl}_{3}$; and (d) local structure of $\mathrm{Zr}$ from the FPMD simulation of the $\mathrm{LiCl}-\mathrm{KCl}$ with $\mathrm{ZrCl}_{4}$

Figure 4.14 shows radial distribution functions (RDFs) of the $\mathrm{LiCl}-\mathrm{KCl}$ with $\mathrm{EuCl}_{3}, \mathrm{CeCl}_{3}, \mathrm{NdCl}_{3}$, and $\mathrm{ZrCl}_{4}$, respectively. In general, the solutes with higher oxidation state make more bonds with $\mathrm{Cl}$ ions and the first peak is greater. However, we observed that the coordination number of $\operatorname{Zr}(4+)$ ion is smaller than the $\mathrm{Eu}(3+), \mathrm{Ce}\left(3^{+}\right)$and $\mathrm{Nd}\left(3^{+}\right)$ions. The coordination number of each solutes $\left(\mathrm{Eu}\left(3^{+}\right), \mathrm{Ce}\left(3^{+}\right), \mathrm{Nd}\left(3^{+}\right)\right.$and $\left.\mathrm{Zr}(4+)\right)$ with surrounding $\mathrm{Cl}$ ions is about 7.2, 7.1, 7.1 and 6.2, respectively. As can be seen in Figure 4.14(d), integral of g(r) shows plateau which represents the strong $\mathrm{Zr}(4+)-\mathrm{Cl}(1-)$ bonds. Typical octahedral coordination geometry between $\mathrm{Zr}$ and $\mathrm{Cl}$ were rigidly maintained during the simulation. 


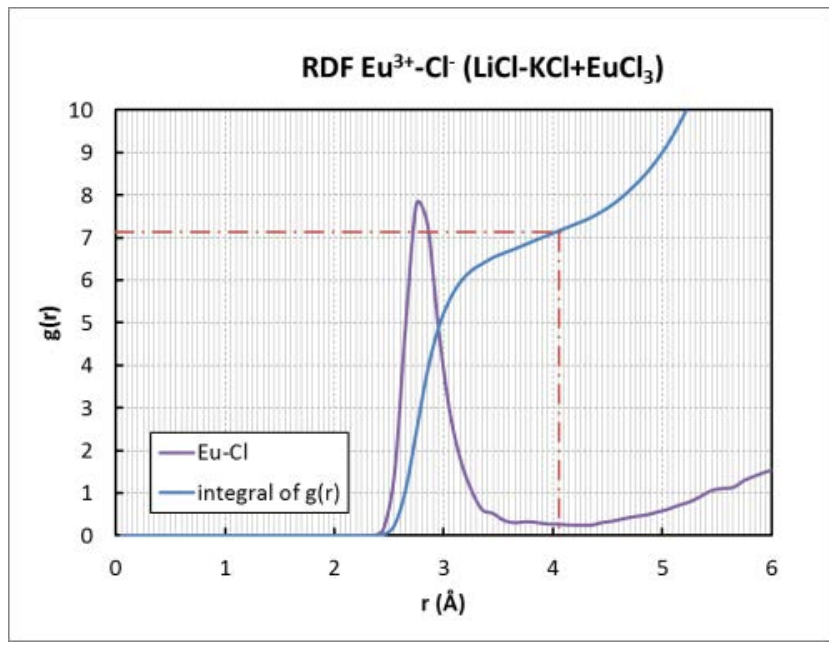

(a)

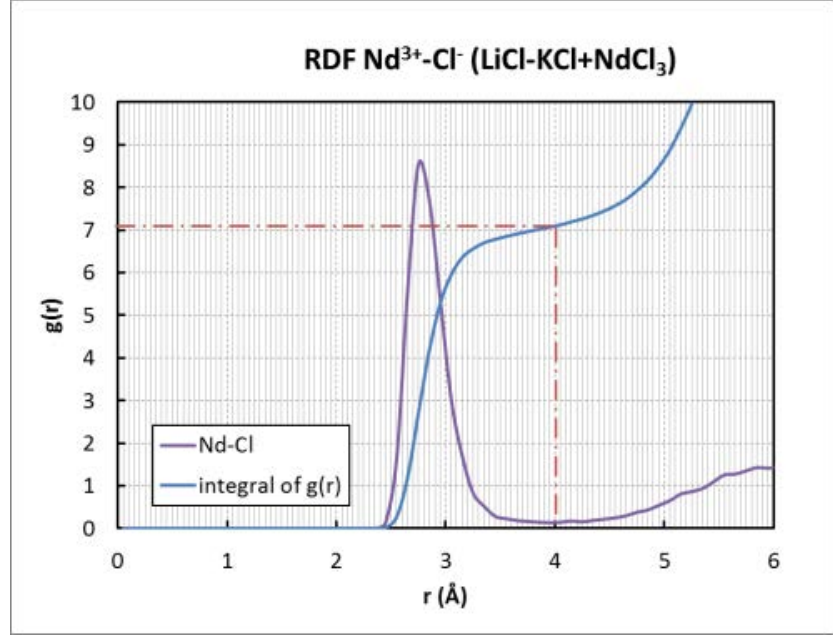

(C)

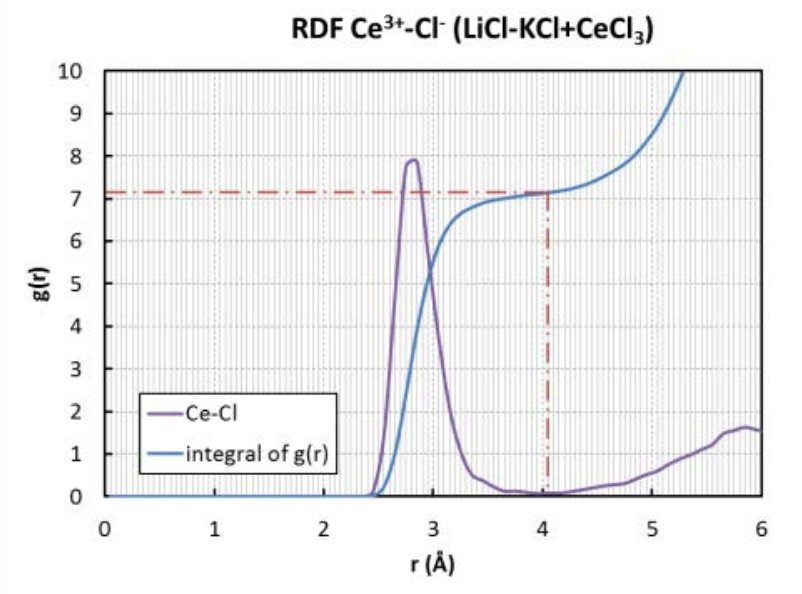

(b)

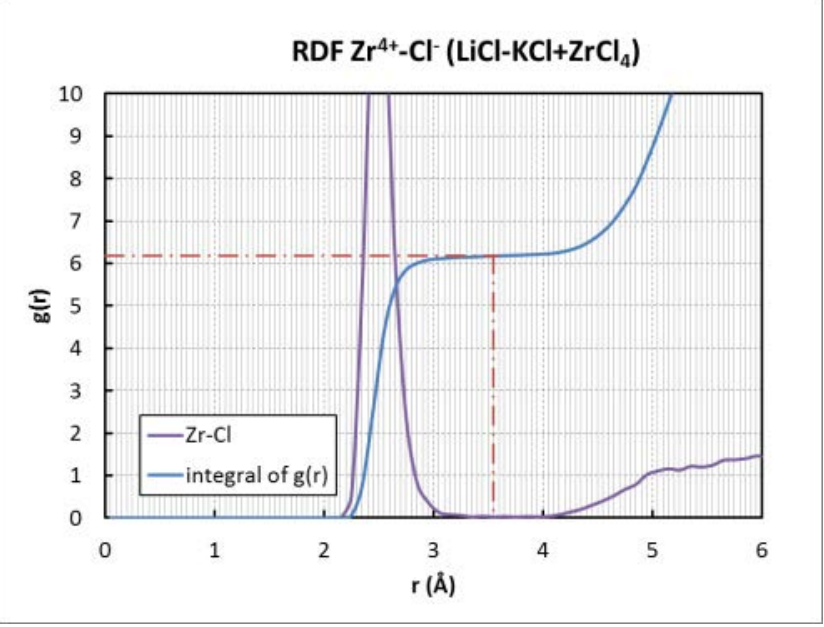

(d)

Figure 4.14 Radial distribution functions (RDF) of the $\mathrm{LiCl}-\mathrm{KCl}$ with (a) $\mathrm{EuCl}_{3}$; (b) $\mathrm{CeCl}_{3}$; (c) $\mathrm{NdCl}_{3}$; (d) $\mathrm{ZrCl}_{4}$ between each solute and $\mathrm{Cl}$ ions.

Table 4.4. First-peak radius and height, and first-shell coordination numbers for solutes and F pairs in LiCl$\mathrm{KCl}$.

\begin{tabular}{|c|c|c|c|}
\hline Ion pair & First-peak radius $(\AA)$ & First-peak height & Coordination number \\
\hline $\mathrm{Sr}^{2+}-\mathrm{F}$ & 2.89 & 6.2 & 6.9 \\
\hline $\mathrm{La}^{3+}-\mathrm{F}$ & 2.85 & 8.5 & 7.4 \\
\hline $\mathrm{U}^{3+}-\mathrm{F}$ & 2.77 & 9.0 & 7.0 \\
\hline $\mathrm{U}^{4+}-\mathrm{F}$ & 2.67 & 10.4 & 7.1 \\
\hline $\mathrm{Eu}^{2+}-\mathrm{F}$ & 2.85 & 6.3 & 6.8 \\
\hline $\mathrm{Eu}^{3+}-\mathrm{F}$ & 2.78 & 7.9 & 7.2 \\
\hline $\mathrm{Nd}^{3+}-\mathrm{F}$ & 2.77 & 8.7 & 7.1 \\
\hline $\mathrm{Ce}^{3+}-\mathrm{F}$ & 2.80 & 8.0 & 6.2 \\
\hline $\mathrm{Zr}^{4+}-\mathrm{F}$ & 2.49 & 13.1 & \\
\hline
\end{tabular}




\subsubsection{Diffusion Coefficient of Solutes}

Self-diffusion coefficients of solutes (Sr, Eu, La, Nd, Ce, U and Zr) in the LiCl-KCl at 773K were analyzed and compiled together. As the accuracy is limited by the number of solute atoms in the cell and simulation time, longer simulation time is needed for more precise answers. Nine separate simulations for 4ps (4,000 timesteps) were conducted for each solute system. The mean-squared displacement (MSD) analysis is used to calculate the diffusion coefficient. The diffusivity values, which were determined from the slope of the MSD for each simulation, are averaged to determine the diffusion coefficient of solute ions for the total simulation time of 36ps.

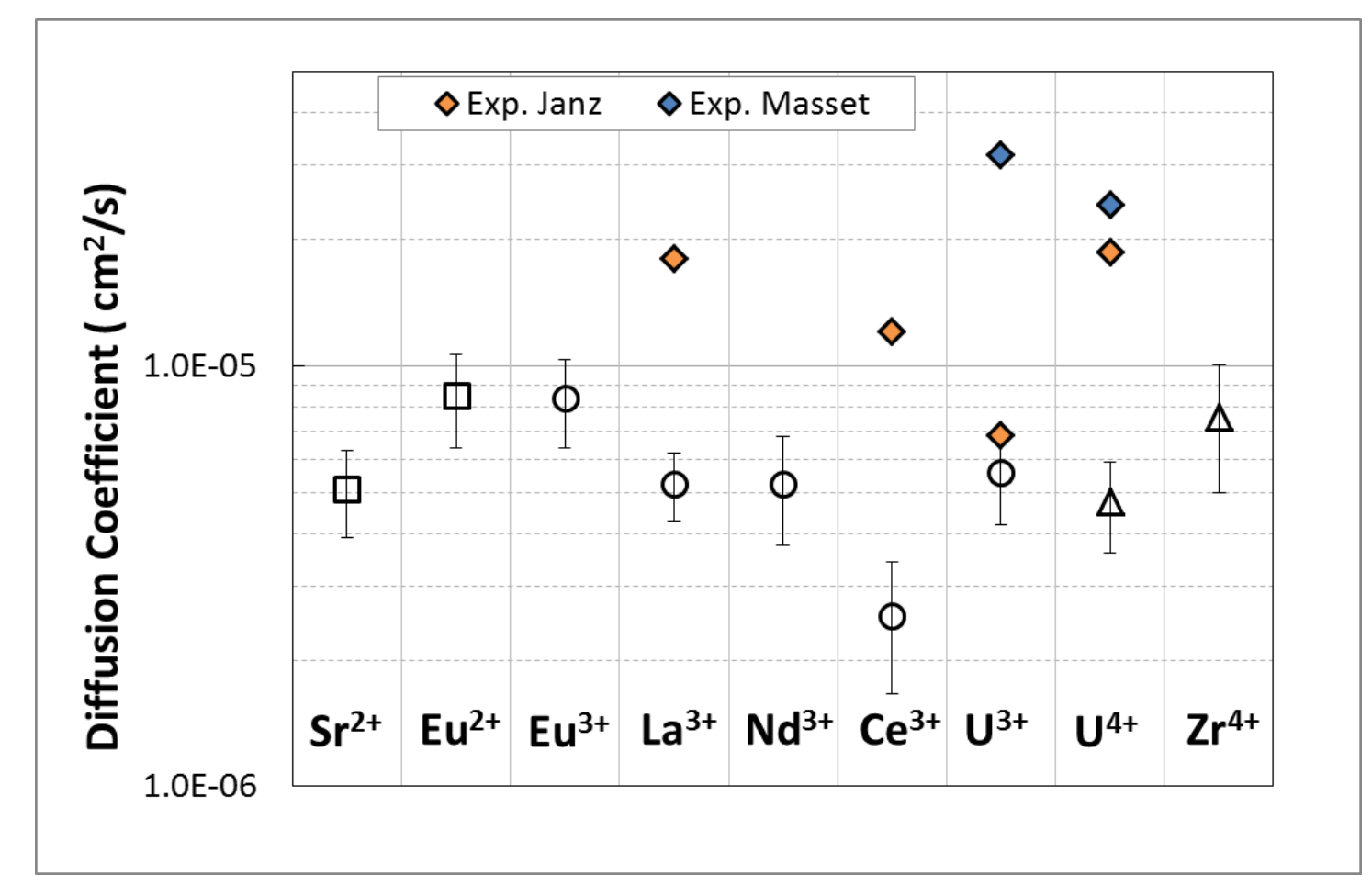

Figure 4.15. Estimated diffusion coefficients of solutes in the $\mathrm{LiCl}-\mathrm{KCl}$ at $773 \mathrm{~K}$. Square marks are 2+, circles are 3+, and triangles are 4+ ions. Experimental diffusion coefficients (diamonds) are also shown for comparison. Error bars represent one sigma standard error of the mean from 9 simulations.

Figure 4.15 shows the estimated diffusion coefficients of various solutes in the $\mathrm{LiCl}-\mathrm{KCl}$ at $773 \mathrm{~K}$ from FPMD simulations (Table 4.5). It includes the experimentally measured diffusion coefficients for $\mathrm{La}, \mathrm{Ce}$ and $\mathrm{U}$ for comparison $[127,128]$. As shown in measured $\mathrm{U}\left(3^{+}\right)$diffusivities from Janz and Masset, experimental data can contain uncertainty. However, calculated diffusion coefficients of solutes are generally about five times smaller than experimental measurements. 
Table 4.5. Estimated diffusion coefficients of solutes in the LiCl-KCl at 773K from FPMD simulations. Error ranges represent one sigma standard error of the mean from 9 simulations.

\begin{tabular}{|c|c|c|c|c|c|c|c|c|c|}
\hline $\begin{array}{c}\mathrm{D} \times 10^{6} \\
\left(\mathrm{~cm}^{2} / \mathrm{sec}\right) \\
\end{array}$ & $\mathrm{Sr}^{2+}$ & $\mathrm{Eu}^{2+}$ & $\mathrm{Eu}^{3+}$ & $\mathrm{La}^{3+}$ & $\mathrm{Nd}^{3+}$ & $\mathrm{Ce}^{3+}$ & $\mathrm{U}^{3+}$ & $\mathrm{U}^{4+}$ & $\mathrm{Zr}^{4+}$ \\
\hline $\mathrm{XCl}_{\mathrm{n}}$ & $\begin{array}{r}5.09 \\
\pm 1.20\end{array}$ & $\begin{array}{r}8.49 \\
\pm 2.12\end{array}$ & $\begin{array}{r}8.37 \\
\pm 1.99\end{array}$ & $\begin{array}{r}5.24 \\
\pm 9.52\end{array}$ & $\begin{array}{r}5.25 \\
\pm 1.52\end{array}$ & $\begin{array}{r}2.54 \\
\pm 0.88\end{array}$ & $\begin{array}{r}5.59 \\
\pm 1.40\end{array}$ & $\begin{array}{r}4.76 \\
\pm 1.16\end{array}$ & $\begin{array}{r}7.53 \\
\pm 2.54\end{array}$ \\
\hline
\end{tabular}

This underestimation of solute diffusivity can potentially be explained by the effect of solute concentration. Martin and Sridharan et al. observed that the diffusion coefficient drops off significantly with lanthanide chloride concentration [129]. The concentration of $\mathrm{LaCl}_{3}$ in our simulation cell with 64 atoms of $\mathrm{LiCl}-\mathrm{KCl}$ is about 120,000 ppm and our data shows excellent agreement with the experimental diffusivity data measured at higher concentration of lanthanide as shown in Figure 4.16. However, the simulations seem to show little or no increase in D with lower concentration, although agreement at lower concentrations of $\mathrm{La}$ is less clear due to large uncertainties in the simulations. The concentration dependence of the diffusivity is an area that wants more detailed study.

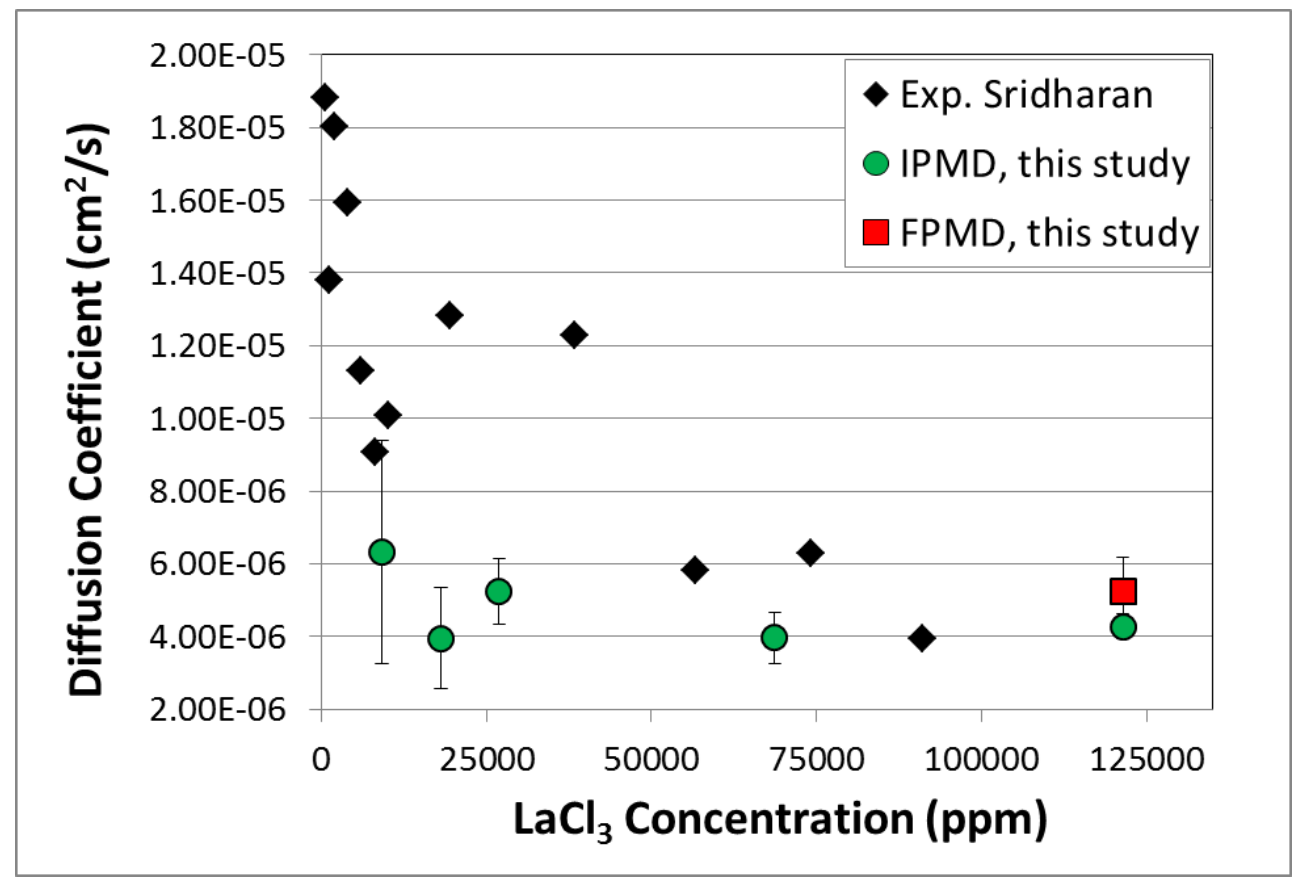

Figure 4.16. Comparison of diffusion coefficient of $\mathrm{La}\left(3^{+}\right)$from FPMD (Red rectangular with standard error) with the experimental measurements [129].

\subsubsection{Standard Redox Potential for Solutes in Molten LiCl-KCl Eutectic Salt}

Partitioning process of spent nuclear fuels in eutectic $\mathrm{LiCl}-\mathrm{KCl}$ has been developed to recycle the spent fuels and reduce the toxicity/volume of disposable nuclear waste. Most countries are now pursuing closed fuel cycle and hence the importance of pyroprocessing research is growing. For the modeling of partitioning process, it is essential to start with accurate data on the thermochemical properties of solutes in molten salt. However, the desired data is often not available, either due to 
there being no experimental studies or the existing studies showing a large scatter due to the different experimental conditions.

For the modeling of solute behavior in molten LiCl-KCl salt, we employed quantum mechanical first principles molecular dynamics (FPMD), which do not rely on experimental input and can generate essential thermokinetic information for solutes. We formulated the equation to estimate the redox potential from the ensemble average of energy from FPMD and a proper choice of reference state. The predicted redox potentials are then compared with measured or theoretical redox potentials that can be predicted from the free energy of formation for pure solid phase chlorides.

We calculated the standard redox potential for various redox couples from FPMD modeling following the general electrochemical referencing approach developed by Nørskov and collaborators [130]. The original target was the redox reaction for $\mathrm{Sr}(\mathrm{II}) / \mathrm{Sr}(0), \mathrm{La}(\mathrm{III}) / \mathrm{La}(0)$, $\mathrm{U}(\mathrm{IV}) / \mathrm{U}(\mathrm{III})$, and $\mathrm{U}(\mathrm{III}) / \mathrm{U}(0)$ couples in molten $\mathrm{LiCl}-\mathrm{KCl}$ eutectic salt at $773 \mathrm{~K}\left(500{ }^{\circ} \mathrm{C}\right)$, which is the operating temperature of the electro-refining system. In addition, we further simulate the systems for the $\mathrm{Li}(\mathrm{I}) / \mathrm{Li}(0), \mathrm{Zr}(\mathrm{IV}) / \mathrm{Zr}(0), \mathrm{Eu}(\mathrm{III}) / \mathrm{Eu}(\mathrm{II}), \mathrm{Eu}(\mathrm{II}) / \mathrm{Eu}(0), \mathrm{Nd}(\mathrm{III}) / \mathrm{Nd}(0)$, and $\mathrm{Ce}(\mathrm{III}) / \mathrm{Ce}(0)$ couples at $773 \mathrm{~K}$ in order to get more data to compare with the measurements and theoretical redox potentials.

We formulated the equation to calculate the redox potential from FPMD based on the redox reaction. For example, corresponding chemical reactions for the redox couple of $\mathrm{U}(\mathrm{IV}) / \mathrm{U}(\mathrm{III})$ is shown below;

$2 \mathrm{UCl}_{4}$ dissolved in salt $=2 \mathrm{UCl}_{3}$ dissolved in salt $+\mathrm{Cl}_{2}(\mathrm{~g})$

Based on the above reactions, we estimate the redox potential for U(IV)/U(III) using Eq. (4.2) [131],

$$
\left\langle V_{\text {redox }}\right\rangle_{U C l_{4} / U C l_{3}}\left(\text { vs. } C l_{2} / C l^{-}\right)=\frac{\left[\left\langle E\left(U C l_{4}+M S\right)\right\rangle-\left\langle E\left(U C l_{3}+M S\right)\right\rangle-0.5 \cdot G\left(C l_{2}\right)\right]+C^{S}}{1 \cdot e}
$$

where $<E>$ is the ensemble average of total energy as calculated from FPMD, $e$ the absolute value of the electron charge, $G\left(\mathrm{Cl}_{2}\right)$ the free energy of a chlorine gas [132], and $C^{S}$ the entropy correction term [133] as shown in Eq. (4.3),

$$
C^{S}=-\left(T \cdot S_{U C l_{4}}^{\text {pure }}-T \cdot S_{U C l_{3}}^{\text {pure }}\right) / F
$$

where $T$ is the temperature and $F$ the Faraday constant. This term compensate the entropy term for the dissolved chlorides because the energy from the FPMD do not include the entropic contributions in it.

In order to obtain a reliable statistical average for the energy of the solute system, nine independent FPMD simulations for 4 8 ps were performed for each solute. All simulations were run with the 64 atoms of pure $\mathrm{LiCl}-\mathrm{KCl}$ simulation cell $(19 \mathrm{Li}, 13 \mathrm{~K}$, and $32 \mathrm{Cl}$ atoms). For the modeling of solute ion (e.g. for $\mathrm{Sr}(\mathrm{II})$ in $\mathrm{LiCl}-\mathrm{KCl}$ ), $\mathrm{SrCl}_{2}$ molecule was introduced into the 64 atoms of $\mathrm{LiCl}-\mathrm{KCl}$ to ionize the $\operatorname{Sr}(0)$ to $\operatorname{Sr}(\mathrm{II})$. 
Figure 4.17 shows the comparison of calculated redox potential of several redox couples in molten $\mathrm{LiCl}-\mathrm{KCl}$ salt at $773 \mathrm{~K}$ by FPMD with the (a) experimentally measured redox potential ( $\mathrm{vs} \mathrm{Cl}_{2} / \mathrm{Cl}^{-}$ reference) and the (b) predicted redox potential for solid form of chlorides [133]. Table 4.6 summarizes the calculated values of redox potential for those couples in molten LiCl-KCl salt at 773K. Due to the scarcity of reliable redox potential measurements for solutes in $\mathrm{LiCl}-\mathrm{KCl}$ at $773 \mathrm{~K}$, only four redox points are compared. As the predicted redox potential is obtained from the chloridizing reaction of pure solid phase substance, the values can be different with the actual measurements of redox reaction in the molten salt. However, these predicted redox values are useful for comparison because they are generally quite close to the potentials measured in the salt.

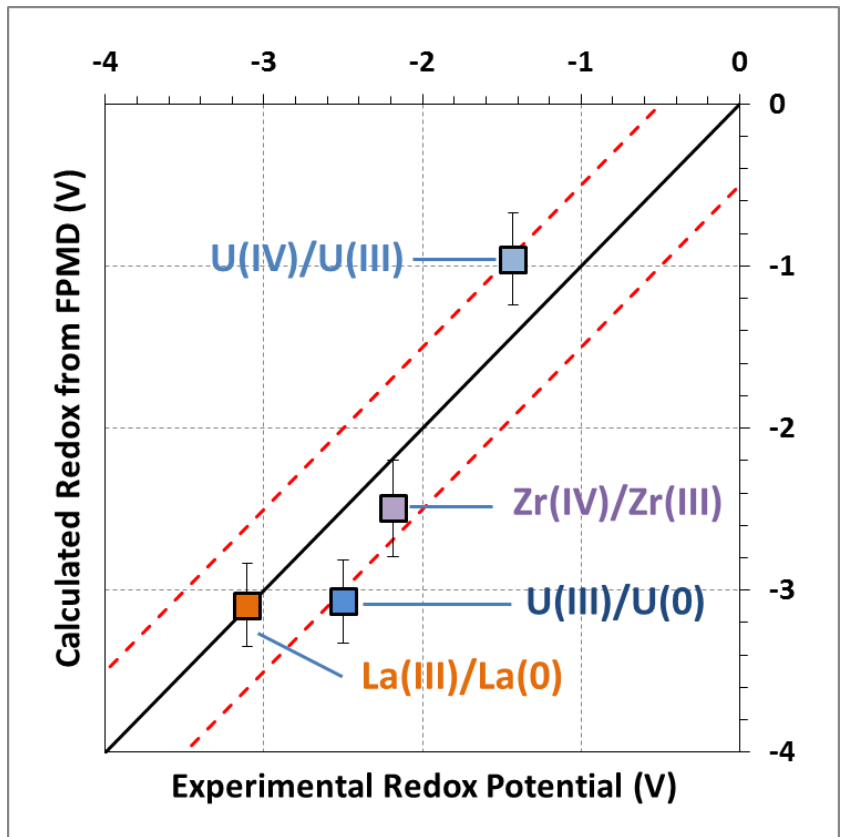

(a)

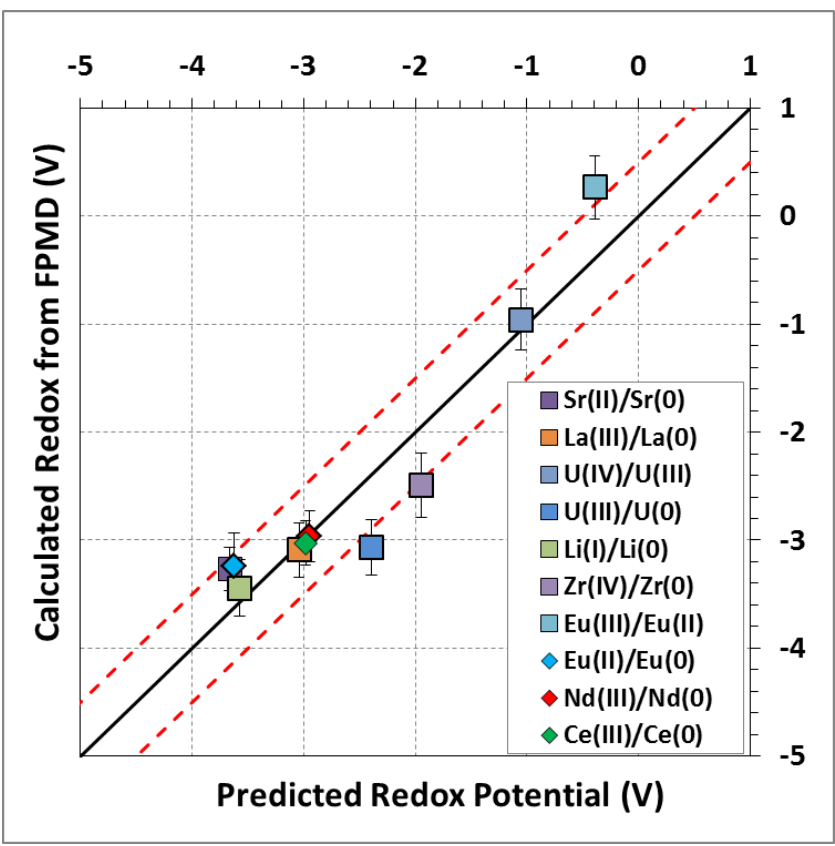

(b)

Figure 4.17 Comparison of calculated redox potential of several redox couples in molten LiCl-KCl salt at 773K by FPMD (y-axis) with the (a) experimental redox potential and the (b) predicted redox potential from the thermodynamic database for pure solid form of chlorides [133]. Red dashed lines are showing $\pm 0.5 \mathrm{~V}$ range from the guide line. The error bars represent the two sigma standard error of the mean.

Although calculated redox potential for solutes are not perfectly matched with the measurements and the prediction, most of the calculated redox potentials are within the range of $\pm 0.5 \mathrm{~V}$ from the experimental data. Various reasons can be proposed to explain the difference between the calculation and references (measurements or prediction), including DFT errors, statistical issues in FPMD, and several approximations that we have made in the approach to keep the study practical. In this approach, we took energy from the FPMD simulations instead of enthalpy in the redox formulation (assuming $\mathrm{P}=0$ ). And it is assumed that the entropy difference between solutes $(\mathrm{X})$ in $\mathrm{LiCl}-\mathrm{KCl}$ and chlorides $\left(\mathrm{XCl}_{\mathrm{n}}\right)$ in $\mathrm{LiCl}-\mathrm{KCl}$ is same with the difference of entropy between solid phase $\mathrm{X}$ and solid phase $\mathrm{XCl}_{\mathrm{n}}$. We believe that further refinement is possible with exploration of additional first-principles exchang-correlation functionals as well as more extensive simulation employing larger simulation cells, longer simulations, more sampling of simulation runs, and running simulation at the most accurate equilibrium volume for solute systems. 
Table 4.6 Calculated redox potential of several redox couples in molten $\mathrm{LiCl}-\mathrm{KCl}$ salt at $773 \mathrm{~K}$ with the predicted redox potential from the thermodynamic database [133].

\begin{tabular}{|c|c|c|c|}
\hline Redox couples & $\begin{array}{c}\text { Prediction [133] } \\
(\mathrm{eV})\end{array}$ & $\begin{array}{c}\text { FPMD (this study) } \\
(\mathrm{eV})\end{array}$ & $\begin{array}{c}\text { 2 Sigma Std. Error } \\
(\mathrm{eV})\end{array}$ \\
\hline $\mathrm{Sr}(\mathrm{II}) / \mathrm{Sr}(0)$ & -3.663 & -3.266 & 0.204 \\
\hline $\mathrm{La}(\mathrm{III}) / \mathrm{La}(0)$ & -3.043 & -3.091 & 0.255 \\
\hline $\mathrm{U}(\mathrm{IV}) / \mathrm{U}(\mathrm{III})$ & -1.056 & -0.958 & 0.284 \\
\hline $\mathrm{U}(\mathrm{III}) / \mathrm{U}(0)$ & -2.393 & -3.069 & 0.258 \\
\hline $\mathrm{Li}(\mathrm{I}) / \mathrm{Li}(0)$ & -3.574 & -3.445 & 0.261 \\
\hline $\mathrm{Zr}(\mathrm{IV}) / \mathrm{Zr}(0)$ & -1.946 & -2.494 & 0.299 \\
\hline $\mathrm{Eu}(\mathrm{III}) / \mathrm{Eu}(\mathrm{II})$ & -0.394 & 0.265 & 0.296 \\
\hline $\mathrm{Eu}(\mathrm{II}) / \mathrm{Eu}(0)$ & -3.625 & -3.241 & 0.312 \\
\hline $\mathrm{Nd}(\mathrm{III}) / \mathrm{Nd}(0)$ & -2.946 & -2.967 & 0.238 \\
\hline $\mathrm{Ce}(\mathrm{III}) / \mathrm{Ce}(0)$ & -2.983 & -3.029 & 0.204 \\
\hline
\end{tabular}




\subsection{Interatomic Potential Development for Sr/La/U Solutes}

We have discovered that the interatomic potential for $\mathrm{La}$ and $\mathrm{U}$ in the $\mathrm{LiCl}-\mathrm{KCl}$ was already developed in the literature [1]. In this project, therefore, we focused on the development of interatomic potential for Sr solute in the $\mathrm{LiCl}-\mathrm{KCl}$.

The force-matching algorithm as implemented in POTFIT [134] was used to generate the interatomic pair potential for $\mathrm{Sr}$ in the molten $\mathrm{LiCl}-\mathrm{KCl}$. The potential has the Tosi-Fumi (or BornHuggins-Meyer) form [135, 136] as shown below:

$U(i, j)=p[1] \exp \left[\frac{p[3]-r_{i j}}{p[2]}\right]-\frac{p[4]}{r_{i j}^{6}}+\frac{p[5]}{r_{i j}^{8}}$

whereby the five parameters are used for each pair potential with the inclusion of electrostatic potential.

\subsubsection{Fitting of Potential Parameters}

As part of the effort to yield a transferable pair potential model for the LiCl-KCl molten salt that incorporates Sr, we generate the parameterized potential model based on the results from the firstprinciple molecular dynamics calculations. In particular, we utilized the potential fitting code (POTFIT) to extract the Hellman-Feynman Cartesian force acting on each atom, the stress tensor on the simulated MD cell and the cohesive energy obtained from the results from $a b$-initio-based equation of state (EOS) calculations. For this purpose, a weighted target function $\mathrm{Z}(\xi)$ was defined and minimized to quantify the deviations of the forces, stresses and energies from the (ab-initiobased) reference values:

$$
\begin{aligned}
& Z(\xi)=Z_{F}(\xi)+Z_{C} \\
& Z_{F}(\xi)=\sum_{k=1}^{m} u_{k}\left(F_{k}(\xi)-F_{k}^{0}\right)^{2} \\
& Z_{C}(\xi)=\sum_{r=1}^{N_{C}} w_{r}\left(A_{k}(\xi)-A_{k}^{0}\right)^{2}
\end{aligned}
$$

where $\mathrm{Z}_{\mathrm{F}}$ represents the contributions of the forces $\mathrm{F}_{\mathrm{k}}$, and $\mathrm{Z}_{\mathrm{C}}$ those of the collective quantities i.e. the total stress tensors and cohesive energies. $F_{k}^{0}$ is the reference component value for the forces on each atom whereas $A_{k}^{0}$ is the reference value for the collective quantities. The force weighting factor $\boldsymbol{u}_{\boldsymbol{k}}$ was set to account for the relative rather than absolute deviations from the reference forces whereas $w$,were the weights of the different collective terms. The optimization process implements a least square minimization module, using a combination of a deterministic conjugate gradient algorithm and a stochastic simulated annealing algorithm. 
As an initial stage of the potential fitting, we implemented a simpler form of Morse potential and utilized the data from both the LiCl-KCl (64 atoms total in each cell) and Sr-doped LiCl-KCl (217 atoms including $1 \mathrm{Sr}$ atom in each simulated MD cell) FPMD simulations. As exemplified in Figure 4.18, there is a very good agreement between the forces (acting on the potassium atoms) calculated from $a b$-initio molecular dynamics and those resulted from the fitting potential obtained by POTFIT.

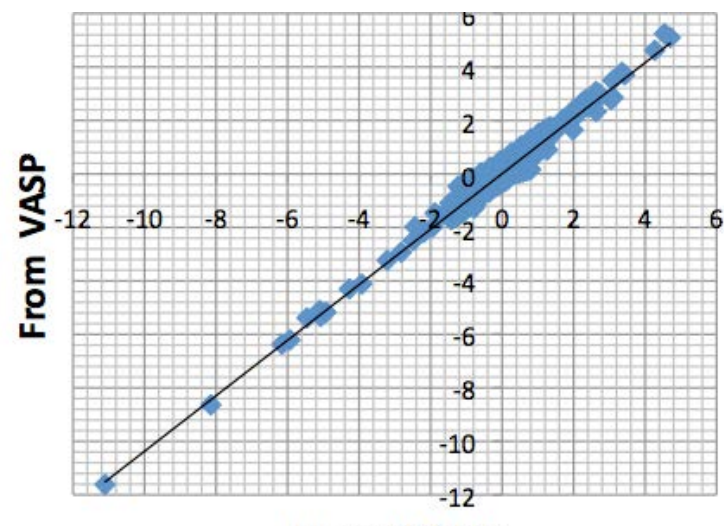

From POTFIT

Figure 4.18. Comparison between Cartesian force components on individual $\mathrm{K}$ atoms (unit is meV/Å).

While there is a reasonable comparison that can be made to the case of $\mathrm{Sr}$ forces as shown in Figure 4.19, due to the limited number of Sr atoms used in the simulated MD cell currently, the matching is not as robust.

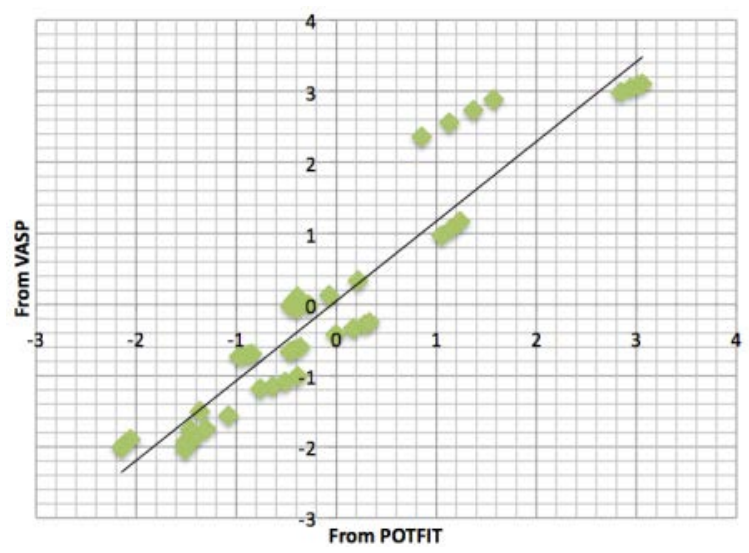

Figure 4.19. Comparison between Cartesian force components on individual Sr atoms (unit is meV/Å).

The preliminary results show that the approach we employed to generate $a b$-initio based potential is quite promising and applicable to the molten salt systems. To improve the Sr potential model, we conducted additional $a b$-initio MD simulations that incorporate higher percentage of Sr dopants to 
better model the Sr-Sr short intercations. In addition, a more realistic interaction potential of the Tosi-Fumi form for the molten salt was explored (see Sec 4.4.2).

\subsubsection{Interatomic Potential for Sr in LiCl-KCl}

We fit a Tosi-Fumi pair potentialfor Sr-X using the previously optimized parameters [1] for the LiCl-KCl molten salt system (Table 4.7). For the validation of Sr potential, IPMD simulation was conducted with the same cell size (67 atoms) of FPMD simulation. For more sampling of Sr in the LiCl-KCl, longer simulation (400ps) was conducted than the FPMD (36ps).

Table 4.7. Parameters for Tosi-Fumi potential for the $\mathrm{LiCl}-\mathrm{KCl}$ system with $\mathrm{Sr}$

\begin{tabular}{|c|c|c|l|l|l|}
\hline & $\mathrm{p} 1$ & \multicolumn{1}{|c|}{$\mathrm{p} 2$} & \multicolumn{1}{c|}{$\mathrm{p} 3$} & \multicolumn{1}{c|}{$\mathrm{p} 4$} & \multicolumn{1}{c|}{$\mathrm{p} 5$} \\
\hline \hline $\mathrm{Li}^{+}-\mathrm{Li}^{+}$ & 0.26091 & 0.33706 & 1.800 & 0.04780 & -0.01673 \\
\hline $\mathrm{Li}^{+}-\mathrm{K}^{+}$ & 0.21767 & 0.33706 & 2.420 & 0.83058 & -0.53546 \\
\hline $\mathrm{Li}^{+}-\mathrm{Cl}$ & 0.17292 & 0.33922 & 2.570 & 1.24886 & -1.50597 \\
\hline $\mathrm{Li}^{+}-\mathrm{Sr}^{2+}$ & 0.10463 & 0.36958 & 3.615 & 1.00938 & -15.67553 \\
\hline $\mathrm{K}^{+}-\mathrm{K}^{+}$ & 0.19015 & 0.33922 & 3.040 & 15.1656 & -14.97603 \\
\hline $\mathrm{K}^{+}-\mathrm{Cl}^{-}$ & 0.13741 & 0.34141 & 3.190 & 29.9606 & -45.56394 \\
\hline $\mathrm{K}^{+}-\mathrm{Sr}^{2+}$ & 0.48670 & 0.22208 & 2.271 & 38.24447 & -19.75263 \\
\hline $\mathrm{Cl}^{-}-\mathrm{Cl}^{-}$ & 0.17412 & 0.34587 & 3.340 & 132.80918 & -1247.52831 \\
\hline $\mathrm{Cl}^{-}-\mathrm{Sr}^{2+}$ & 0.66094 & 0.34694 & 2.851 & 4.06901 & -29.72559 \\
\hline $\mathrm{Sr}^{2+}-\mathrm{Sr}^{2+}$ & 0.17457 & 0.26387 & 2.842 & 100.29902 & -182.67930 \\
\hline
\end{tabular}

The comparison of Sr-X RDFs between IPMD (LAMMPS) and FPMD (VASP) are shown in Figure 4.20. Solid and dashed lines are RDFs obtained from the IPMD and FPMD, respectively. RDFs show a reasonable agreement between the results from the FPMD and the IPMD. As only one $\mathrm{Sr}$ atom exists in the cell, the RDFs between Sr-X are not smooth, especially the RDFs from the FPMD (dashed). As can be seen in Figure 4.20, even the RDF between $\mathrm{Li}$ and $\mathrm{Li}$ (19 atoms in the cell) is not perfectly superimposed onto each other between the IPMD and the FPMD. The disagreements may be caused by the small differences in the cell size which were used in each IPMD (lattice

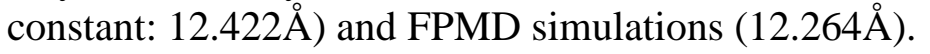




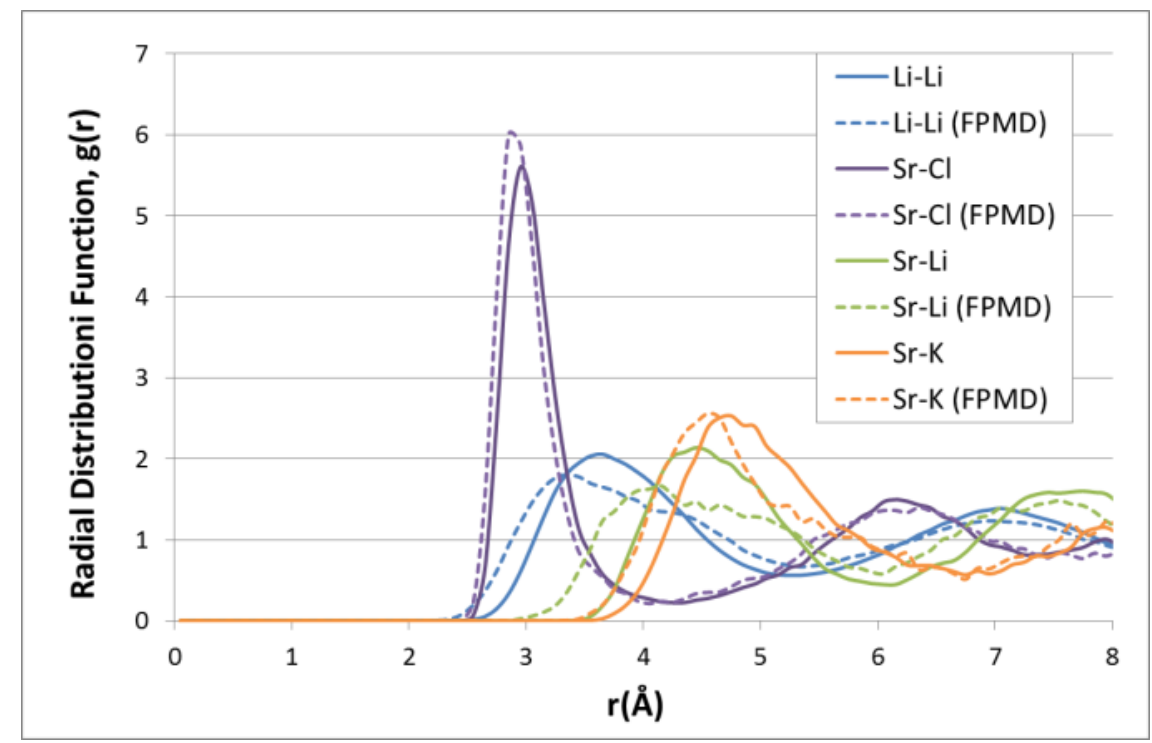

Figure 4.20. Comparison of RDFs of Sr-X showing a reasonable agreement between the results from the IPMD (solid line) and the FPMD (dashed line)

We further compared the self-diffusion coefficients of Li, K, Cl and solute Sr ions (Figure 4.21). Calculated IPMD diffusivities are shown as square marks and the diffusivities from FPMD are shown as open circles with standard errors. As longer simulations were conducted, the diffusivities from IPMD have smaller errors than the diffusivities from FPMD. The comparison of diffusivities shows a reasonable agreement.

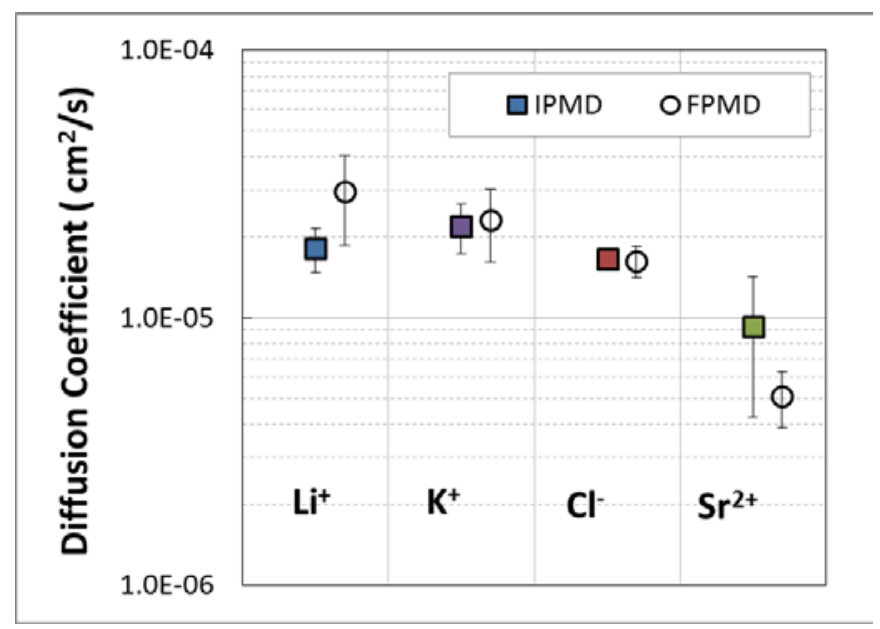

Figure 4.21. Comparison of estimated diffusion coefficients from IPMD (squares) and the FPMD (circles). The error represents the one sigma standard error

We should note that, as has also been found by other studies, the $\mathrm{p}$ [4] and $\mathrm{p}$ [5] parameters may be sensitive to the concentration of $\mathrm{LiCl}-\mathrm{KCl}$ molten salt as well as the concentration of the solutes and the sizes of the simulation cells. 


\section{References}

1. Salanne, M., et al., Calculation of Activities of Ions in Molten Salts with Potential Application to the Pyroprocessing of Nuclear Waste. The Journal of Physical Chemistry B, 2008. 112(4): p. 1177-1183.

2. Kato, T., et al., Separation behaviors of actinides from rare-earths in molten salt electrorefining using saturated liquid cadmium cathode. J. Nucl. Mater., 2006. 357(1-3): p. 105-114.

3. Katz, J.J., G.T. Seaborg, and L.R. Morss, The Chemistry of the Actinide Elements (II ed.). Vol. 1. 1986, New York: Chapman and Hall.

4. Salanne, M., et al., Calculation of Activities of Ions in Molten Salts with Potential Application to the Pyroprocessing of Nuclear Waste. J. Phys. Chem. B, 2008. 112(4): p. 1177-1183.

5. Sakamura, Y., et al., Separation of actinides from rare earth elements by electrorefining in LiCl-KCl eutectic salt. Journal of Nuclear Science and Technology, 1998. 35: p. 49.

6. Li, S.X., et al., Actinide Recovery Experiments with Bench-Scale Liquid Cadmium Cathode in Real Fission Product-Laden Molten Salt. Nuclear Technology, 2009. 165: p. 190.

7. $\quad$ Masset, P., et al., Thermochemical properties of lanthanides $(L n=L a, N d)$ and actinides $(A n=U, N p, P u, A m)$ in the molten LiCl-KCl eutectic. J. Nucl. Mater., 2005. 344(1-3): p. 173-179.

8. Rollet, A.-L. and M. Salanne, Studies of the local structures of molten metal halides. Annual Reports Section "C" (Physical Chemistry), 2011. 107(0): p. 88-123.

9. Pascal, T.A., et al., On the absolute thermodynamics of water from computer simulations: A comparison of first-principles molecular dynamics, reactive and empirical force fields. J. Chem. Phys., 2012. 137(24): p. 244507-7.

10. Debnath, A., et al., Entropy and dynamics of water in hydration layers of a bilayer. J. Chem. Phys., 2010. 133(17): p. 174704-14.

11. Schilling, T. and F. Schmid, Computing absolute free energies of disordered structures by molecular simulation. J. Chem. Phys., 2009. 131(23): p. 231102-4.

12. Lin, S.-T., P.K. Maiti, and W.A. Goddard, Two-Phase Thermodynamic Model for Efficient and Accurate Absolute Entropy of Water from Molecular Dynamics Simulations. J. Phys. Chem. B, 2010. 114(24): p. 81918198.

13. Huang, S.-N., et al., Absolute Entropy and Energy of Carbon Dioxide Using the Two-Phase Thermodynamic Model. J. Chem. Theory Comput., 2011. 7(6): p. 1893-1901.

14. Ovchinnikov, V., M. Cecchini, and M. Karplus, A Simplified Confinement Method for Calculating Absolute Free Energies and Free Energy and Entropy Differences. J. Phys. Chem. B, 2013. 117(3): p. 750-762.

15. Teweldeberhan, A.M. and S.A. Bonev, Structural and thermodynamic properties of liquid Na-Li and Ca-Li alloys at high pressure. Phys. Rev. B, 2011. 83(13): p. 134120.

16. Nayhouse, M., et al., Simulation of fluid-solid coexistence via thermodynamic integration using a modified cell model. J. Phys.: Condens. Matter, 2012. 24: p. 155101.

17. Anwar, J., D. Frenkel, and M.G. Noro, Calculation of the melting point of NaCl by molecular simulation. J. Chem. Phys., 2003. 118(2): p. 728-735. 
18. Rodrigues, P.C.R. and F.M.S.S. Fernandes, Phase diagrams of alkali halides using two interaction models: A molecular dynamics and free energy study. J. Chem. Phys., 2007. 126(2): p. 024503-10.

19. Habershon, S. and D.E. Manolopoulos, Thermodynamic integration from classical to quantum mechanics. J. Chem. Phys., 2011. 135(22): p. 224111-6.

20. Katayama, Y., et al., A first-order liquid-liquid phase transition in phosphorus. Nature, 2000. 403(6766): p. 170-173.

21. Mallamace, F., et al., Evidence of the existence of the low-density liquid phase in supercooled confined water. Proc. Nat. Acad. Sci., 2007. 104: p. 424.

22. Beye, M., et al., The liquid-liquid phase transition in silicon revealed by snapshots of valence electrons. Proc. Nat. Acad. Sci., 2010. 107(39): p. 16772-16776.

23. Greaves, G.N., et al., Detection of First-Order Liquid/Liquid Phase Transitions in Yttrium Oxide-Aluminum Oxide Melts. Science, 2008. 322(5901): p. 566-570.

24. Mei, X. and J. Eapen, Dynamic transitions in molecular dynamics simulations of supercooled silicon. Phys. Rev. B, 2013. 87: p. 134206.

25. Eapen, J., J. Li, and S. Yip, Beyond the Maxwell limit: Thermal conduction in nanofluids with percolating fluid structures. Phys. Rev. E, 2007. 76(6): p. 062501.

26. Lin, S.-T., M. Blanco, and W.A. Goddard, The two-phase model for calculating thermodynamic properties of liquids from molecular dynamics: Validation for the phase diagram of Lennard-Jones fluids. J. Chem. Phys., 2003. 119: p. 11792.

27. Pascal, T.A., S.-T. Lin, and W.A. Goddard, Thermodynamics of liquids: standard molar entropies and heat capacities of common solvents from 2PT molecular dynamics. Phys. Chem. Chem. Phys., 2011. 13(1): p. 169181.

28. Zhang, C., L. Spanu, and G. Galli, Entropy of Liquid Water from Ab Initio Molecular Dynamics. J. Phys. Chem. B, 2011. 115(48): p. 14190-14195.

29. Lai, P.-K., C.-M. Hsieh, and S.-T. Lin, Rapid determination of entropy and free energy of mixtures from molecular dynamics simulations with the two-phase thermodynamic model. Phys. Chem. Chem. Phys., 2012. 14(43): p. 15206-15213.

30. $\quad$ Pascal, T.A., et al., Role of Specific Cations and Water Entropy on the Stability of Branched DNA Motif Structures. J. Phys. Chem. B, 2012. 116(40): p. 12159-12167.

31. Carnahan, N.F. and K.E. Starling, Thermodynamic Properties of a Rigid-Sphere Fluid. J. Chem. Phys., 1970. 53(2): p. 600-603.

32. Chemla, M. and I. Okada, Ionic mobilities of monovalent cations in molten salt mixtures. Electrochimica Acta, 1990. 35(11-12): p. 1761-1776.

33. Okada, I., The Chemla effect ---from the separation of isotopes to the modeling of binary ionic liquids. Journal of Molecular Liquids, 1999. 83(1-3): p. 5-22.

34. Ribeiro, M.C.C., On the Chemla effect in molten alkali nitrates. The Journal of Chemical Physics, 2002. 117(1): p. 266-276.

35. Ribeiro, M.C.C., Chemla Effect in Molten LiCl/KCl and LiF/KF Mixtures. J. Phys. Chem. B, 2003. 107(18): p. 4392-4402. 
36. Morgan, B. and P.A. Madden, Ion mobilities and microscopic dynamics in liquid (Li,K)Cl. The Journal of Chemical Physics, 2004. 120(3): p. 1402-1413.

37. Hutchinson, F., et al., The "ionic" to "molecular" transitions in AlCl[sub 3] and FeCl[sub 3] as predicted by an ionic interaction model. The Journal of Chemical Physics, 1999. 110(12): p. 5821-5830.

38. East, A.L.L. and J. Hafner, Short-Range Order in Liquid Aluminum Chloride: Ab Initio Molecular Dynamics Simulations and Quantum-Chemical Calculations. The Journal of Physical Chemistry B, 2007. 111(19): p. 5316-5321.

39. deGroot, S.R. and P. Mazur, Nonequilibrium thermodynamics1984, New York: Dover Publications, Inc.

40. Hanley, H.J.M., Transport Phenomena in Fluids, ed. H.J.M. Hanley1969, New York: Marcel Dekker.

41. Sarou-Kanian, V., et al., Diffusion coefficients and local structure in basic molten fluorides: in situ NMR measurements and molecular dynamics simulations. Physical Chemistry Chemical Physics, 2009. 11(48): p. 11501-11506.

42. Rollet, A.-L., V. Sarou-Kanian, and C. Bessada, Measuring Self-Diffusion Coefficients up to 1500 K: A Powerful Tool to Investigate the Dynamics and the Local Structure of Inorganic Melts. Inorganic Chemistry, 2009. 48(23): p. 10972-10975.

43. Wambui Mutoru, J. and A. Firoozabadi, Form of multicomponent Fickian diffusion coefficients matrix. The Journal of Chemical Thermodynamics, 2011. 43(8): p. 1192-1203.

44. $\quad$ Curtiss, C.F. and R.B. Bird, Multicomponent Diffusion. Industrial \& Engineering Chemistry Research, 1999. 38(7): p. 2515-2522.

45. Krishna, R. and J.A. Wesselingh, The Maxwell-Stefan approach to mass transfer. Chemical Engineering Science, 1997. 52(6): p. 861-911.

46. Bothe, D., On the Maxwell-Stefan Approach to Multicomponent Diffusion Parabolic Problems, J. Escher, et al., Editors. 2011, Springer Basel. p. 81-93.

47. Liu, X., T.J.H. Vlugt, and A. Bardow, Predictive Darken Equation for Maxwell-Stefan Diffusivities in Multicomponent Mixtures. Industrial \& Engineering Chemistry Research, 2011. 50(17): p. 10350-10358.

48. Bardow, A., et al., Prediction of multicomponent mutual diffusion in liquids: Model discrimination using NMR data. Fluid Phase Equilibria, 2009. 278(1-2): p. 27-35.

49. Guevara-Carrion, G., J. Vrabec, and H. Hasse, Prediction of Transport Properties of Liquid Ammonia and Its Binary Mixture with Methanol by Molecular Simulation. International Journal of Thermophysics, 2012. 33(3): p. 449-468.

50. Rehfeldt, S. and J. Stichlmair, Measurement and prediction of multicomponent diffusion coefficients in four ternary liquid systems. Fluid Phase Equilibria, 2010. 290(1-2): p. 1-14.

51. Liu, X., T.J.H. Vlugt, and A. Bardow, Maxwell-Stefan diffusivities in liquid mixtures: Using molecular dynamics for testing model predictions. Fluid Phase Equilibria, 2011. 301(1): p. 110-117.

52. Annunziata, O., D. Buzatu, and J.G. Albright, Protein Diffusiophoresis and Salt Osmotic Diffusion in Aqueous Solutions. The Journal of Physical Chemistry B, 2012.

53. Kushner, M.J., Mechanisms for Power Deposition in Ar/SiH4 Capacitively Coupled RF Discharges. Plasma Science, IEEE Transactions on, 1986. 14(2): p. 188-196. 
54. Liu, X., A. Bardow, and T.J.H. Vlugt, Multicomponent Maxwell-Stefan Diffusivities at Infinite Dilution. Industrial \& Engineering Chemistry Research, 2011. 50(8): p. 4776-4782.

55. Krishna, R. and J.M. van Baten, The Darken Relation for Multicomponent Diffusion in Liquid Mixtures of Linear Alkanes: An Investigation Using Molecular Dynamics (MD) Simulations. Industrial \& Engineering Chemistry Research, 2005. 44(17): p. 6939-6947.

56. Liu, X., et al., Fick Diffusion Coefficients of Liquid Mixtures Directly Obtained From Equilibrium Molecular Dynamics. The Journal of Physical Chemistry B, 2011. 115(44): p. 12921-12929.

57. Liu, X., et al., Fick Diffusion Coefficients in Ternary Liquid Systems from Equilibrium Molecular Dynamics Simulations. Industrial \& Engineering Chemistry Research, 2012. 51(30): p. 10247-10258.

58. Kirkwood, J.G. and F.P. Buff, The Statistical Mechanical Theory of Solutions. I. J. Chem. Phys., 1951. 19(6): p. 774-777.

59. Wheeler, D.R. and J. Newman, Molecular Dynamics Simulations of Multicomponent Diffusion. 1. Equilibrium Method. The Journal of Physical Chemistry B, 2004. 108(47): p. 18353-18361.

60. $\quad$ Onsager, L., Reciprocal Relations in Irreversible Processes. I. Physical Review, 1931. 37(4): p. 405.

61. $\quad$ Onsager, L., Reciprocal Relations in Irreversible Processes. II. Physical Review, 1931. 38(12): p. 2265.

62. Kraaijeveld, G. and J.A. Wesselingh, Negative Maxwell-Stefan diffusion coefficients. Industrial \& Engineering Chemistry Research, 1993. 32(4): p. 738-742.

63. Todorov, I.T. and W. Smith, DL POLY 3: the CCP5 national UK code for molecular-dynamics simulations. Phil. Trans. R. Soc. Lond. A, 2004. 362: p. 1835.

64. Tosi, M.P. and F.G. Fumi, Ionic sizes and born repulsive parameters in the NaCl-type alkali halides-II: The generalized Huggins-Mayer form. J. Phys. Chem. Solids, 1964. 25(1): p. 45-52.

65. Lantelme, F. and P. Turq, Ionic dynamics in the LiCl--KCl system at liquid state. J. Chem. Phys., 1982. 77(6): p. 3177-3187.

66. Salanne, M., et al., Intermediate range chemical ordering of cations in simple molten alkali halides. J. Phys. Condens. Matter, 2008. 20: p. 332101.

67. Ohtori, N., M. Salanne, and P.A. Madden, Calculations of the thermal conductivities of ionic materials by simulation with polarizable interaction potentials. J. Chem. Phys., 2009. 130(10): p. 104507-13.

68. Salanne, M. and P.A. Madden, Polarization effects in ionic solids and melts. Mol. Phys., 2011. 109(19): p. 2299-2315.

69. Galamba, N., C.A.N.d. Castro, and J.F. Ely, Thermal conductivity of molten alkali halides from equilibrium molecular dynamics simulations. J. Chem. Phys., 2004. 120: p. 8676.

70. Rapaport, D.C., The Art of Molecular Dynamics Simulation, II ed.2004, Cambridge: Cambridge University Press.

71. Standart, G.L., R. Taylor, and R. Krishna, The Maxwell-Stefan formulation of irreversible thermodynamics for simultaneous heat and mass transfer. Chemical Engineering Communications, 1979. 3(4-5): p. 277-289.

72. Matuszak, D. and M.D. Donohue, Inversion of multicomponent diffusion equations. Chemical Engineering Science, 2005. 60(15): p. 4359-4367. 
73. McQuarrie, D.A., Statistical Mechanics 2000, California: University Science Books.

74. Berens, P.H., et al., Thermodynamics and quantum corrections from molecular dynamics for liquid water. J. Chem. Phys., 1983. 79(5): p. 2375-2389.

75. Boon, J.P. and S. Yip, Molecular Hydrodynamics1991, New York: Dover.

76. Shannon, R.D., Revised effective ionic radii and systematic studies of interatomic distances in halides and chalcogenides. Acta Cryst., 1976. A32: p. 751.

77. $\quad$ Plimpton, S.J., Fast Parallel Algorithms for Short-Range Molecular Dynamics. J. Comp. Phys., 1995. 117: p. $1-19$.

78. $\quad$ NIST, http://webbook.nist.gov/chemistry/, 2013.

79. Chakraborty, B., J. Wang, and J. Eapen, Multicomponent diffusion in molten LiCl-KCl: Dynamical correlations and divergent Maxwell-Stefan diffusivities. Phys. Rev. E, 2013. 87(5): p. 052312.

80. Willit, J.L., W.E. Miller, and J.E. Battles, ELECTROREFINING OF URANIUM AND PLUTONIUM - A LITERATURE-REVIEW. Journal of Nuclear Materials, 1992. 195(3): p. 229-249.

81. Sakamura, Y., et al., Separation of actinides from rare earth elements by electrorefining in LiCl-KCl eutectic salt. Journal of Nuclear Science and Technology, 1998. 35(1): p. 49-59.

82. Sakamura, Y., et al., Measurement of standard potentials of actinides (U, Np, Pu, Am) in LiCl-KCl eutectic salt and separation of actinides from rare earths by electrorefining. Journal of Alloys and Compounds, 1998. 271: p. 592-596.

83. Li, S.X., et al., ACTINIDE RECOVERY EXPERIMENTS WITH BENCH-SCALE LIQUID CADMIUM CATHODE IN REAL FISSION PRODUCT-LADEN MOLTEN SALT. Nuclear Technology, 2009. 165(2): p. 190-199.

84. Yamada, D., et al., Diffusion behavior of actinide and lanthanide elements in molten salt for reductive extraction. Journal of Alloys and Compounds, 2007. 444: p. 557-560.

85. Eun, H.C., et al., Recycling of LiCl-KCl eutectic based salt wastes containing radioactive rare earth oxychlorides or oxides. Journal of Nuclear Materials, 2012. 420(1-3): p. 548-553.

86. Vandarkuzhali, S., et al., Electrochemical behaviour of LaCl3 at tungsten and aluminium cathodes in LiCl-KCl eutectic melt. Electrochimica Acta, 2012. 59: p. 245-255.

87. Aguado, A. and P.A. Madden, Molecular dynamics simulations of the liquid-vapor interface of a molten salt. III. Size asymmetry effects and binary mixtures. Journal of Chemical Physics, 2002. 117(16): p. 7659-7668.

88. Lantelme, F. and P. Turq, IONIC DYNAMICS IN THE LICL-KCL SYSTEM AT LIQUID-STATE. Journal of Chemical Physics, 1982. 77(6): p. 3177-3187.

89. Caccamo, C. and M. Dixon, MOLTEN ALKALI-HALIDE MIXTURES - A MOLECULAR-DYNAMICS STUDY OF LI-KCL MIXTURES. Journal of Physics C-Solid State Physics, 1980. 13(10): p. 1887-1900.

90. Ribeiro, M.C.C., Chemla effect in molten LiCl/KCl and LiF/KF mixtures. Journal of Physical Chemistry B, 2003. 107(18): p. 4392-4402.

91. Mendes, E., et al., On the electrochemical formation of Pu-Al alloys in molten LiCl-KCl. Journal of Nuclear Materials, 2012. 420(1-3): p. 424-429. 
92. Salanne, M., et al., Calculation of activities of ions in molten salts with potential application to the pyroprocessing of nuclear waste. Journal of Physical Chemistry B, 2008. 112(4): p. 1177-1183.

93. Cristiglio, V., et al., Local structure of liquid $\mathrm{CaAl}(2) \mathrm{O}(4)$ from ab initio molecular dynamics simulations. Journal of Non-Crystalline Solids, 2008. 354(47-51): p. 5337-5339.

94. Sun, N., et al., First principles molecular dynamics simulations of diopside (CaMgSi2O6) liquid to high pressure. Geochimica Et Cosmochimica Acta, 2011. 75(13): p. 3792-3802.

95. Karki, B.B. and L.P. Stixrude, Viscosity of MgSiO3 Liquid at Earth's Mantle Conditions: Implications for an Early Magma Ocean. Science, 2010. 328(5979): p. 740-742.

96. Woodward, C., et al., Ab initio simulations of molten Ni alloys. Journal of Applied Physics, 2010. 107(11).

97. Zahn, S. and B. Kirchner, Validation of dispersion-corrected density functional theory approaches for ionic liquid systems. Journal of Physical Chemistry A, 2008. 112(36): p. 8430-8435.

98. Hazebroucq, S., et al., Density-functional-based molecular-dynamics simulations of molten salts. Journal of Chemical Physics, 2005. 123(13).

99. Klix, A., A. Suzuki, and T. Terai, Study of tritium migration in liquid Li2BeF4 with ab initio molecular dynamics. Fusion Engineering and Design, 2006. 81(1-7): p. 713-717.

100. Kresse, G. and J. Hafner, Ab-initio molecular-dynamics for liquid-metals. Physical Review B, 1993. 47(1): p. 558-561.

101. Martínez, L., et al., PACKMOL: A package for building initial configurations for molecular dynamics simulations. Journal of Computational Chemistry, 2009. 30(13): p. 2157-2164.

102. Plimpton, S., FAST PARALLEL ALGORITHMS FOR SHORT-RANGE MOLECULAR-DYNAMICS. Journal of Computational Physics, 1995. 117(1): p. 1-19.

103. Berendsen, H.J.C., et al., MOLECULAR-DYNAMICS WITH COUPLING TO AN EXTERNAL BATH. Journal of Chemical Physics, 1984. 81(8): p. 3684-3690.

104. Kresse, G. and J. Furthmüller, Efficiency of ab-initio total energy calculations for metals and semiconductors using a plane-wave basis set. Computational Materials Science, 1996. 6(1): p. 15-50.

105. Kresse, G. and J. Furthmüller, Efficient iterative schemes for ab initio total-energy calculations using a planewave basis set. Physical Review B, 1996. 54(16): p. 11169-11186.

106. Nosé, S., A UNIFIED FORMULATION OF THE CONSTANT TEMPERATURE MOLECULAR-DYNAMICS METHODS. Journal of Chemical Physics, 1984. 81(1): p. 511-519.

107. Grimme, S., Semiempirical GGA-type density functional constructed with a long-range dispersion correction. Journal of Computational Chemistry, 2006. 27(15): p. 1787-1799.

108. Alexopoulos, C., A comprehensive review of methods for simulation output analysis, in Proceedings of the 38th conference on Winter simulation2006, Winter Simulation Conference: Monterey, California. p. 168-178.

109. Murnaghan, F.D., The Compressibility of Media under Extreme Pressures. Proceedings of the National Academy of Sciences, 1944. 30(9): p. 244-247.

110. Allen, M.P. and D.J. Tildesley, Computer Simulation of Liquids1987: Clarendon Press, Oxford 
111. Croteau, T. and G.N. Patey, Structures and rearrangements of LiCl clusters. Journal of Chemical Physics, 2006. 124(24).

112. Taylor, J.R., An Introduction to Error Analysis: The Study of Uncertainties in Physical Measurements1997: University Science Books; 2nd edition (March 10, 1997). 327 pages.

113. Rapaport, D.C., The Art of Molecular Dynamics. 2nd ed2004. 564.

114. Perdew, J.P., et al., Restoring the density-gradient expansion for exchange in solids and surfaces. Physical Review Letters, 2008. 100(13).

115. Csonka, G.I., et al., Assessing the performance of recent density functionals for bulk solids. Physical Review B, 2009. 79(15).

116. Janz, G.J., et al., Physical Properties Data Compilations Relevant to Energy Storage. II. Molten Salts: Data on Single and Multi-Component Salt Systems, 1979, National Bureau of Standards: U.S. Department of Commerce.

117. DeHoff, R.T., Thermodynamics in Materials Science, Second Edition2006, University of Florida, Gainesville, USA: CRC Press. 624.

118. Garai, J. and A. Laugier, The temperature dependence of the isothermal bulk modulus at 1 bar pressure. Journal of Applied Physics, 2007. 101(2): p. 023514-4.

119. Kohler, U., P.G. Johannsen, and W.B. Holzapfel, Equation-of-state data for CsCl-type alkali halides. Journal of Physics-Condensed Matter, 1997. 9(26): p. 5581-5592.

120. Van Artsdalen, E.R. and I.S. Yaffe, Electrical Conductance and Density of Molten Salt Systems: KCl-LiCl, $\mathrm{KCl}-\mathrm{NaCl}$ and KCl-KI. The Journal of Physical Chemistry, 1955. 59(2): p. 118-127.

121. Ito, H., Y. Hasegawa, and Y. Ito, Densities of eutectic mixtures of molten alkali chlorides below $673 \mathrm{~K}$. Journal of Chemical and Engineering Data, 2001. 46(5): p. 1203-1205.

122. Lenke, R., Uebelhac.W, and A. Klemm, SELF-DIFFUSSION IN MOLTEN LICL. Zeitschrift Fur Naturforschung Section a-a Journal of Physical Sciences, 1973. 28(6): p. 881-884.

123. Morgan, B. and P.A. Madden, Ion mobilities and microscopic dynamics in liquid (Li,K)Cl. Journal of Chemical Physics, 2004. 120(3): p. 1402-1413.

124. Janz, G.J., Molten Salts Handbook1967: Academic Press.

125. FactSage. KCl-LiCl Phase Diagram from FTsalt databases. Available from: http://www.crct.polymtl.ca/fact/phase_diagram.php?file=KCl-LiCl.jpg\&dir=FTsalt

126. Humphrey, W., A. Dalke, and K. Schulten, VMD: Visual molecular dynamics. Journal of Molecular Graphics, 1996. 14(1): p. 33-38.

127. Masset, P., et al., Electrochemistry of Uranium in Molten LiCl-KCl Eutectic. Journal of The Electrochemical Society, 2005. 152(6): p. A1109-A1115.

128. Janz, G.J. and N.P. Bansal, Molten Salts Data: Diffusion Coefficients in Single and Multi-Component Salt Systems. Journal of Physical and Chemical Reference Data, 1982. 11(3): p. 505-693.

129. Martin, S., et al. Physical and Electrochemical Properties of Molten LiCl-KCl Salt for Used Fuel Reprocessing. in International Pyroprocessing Research Conference. 2012. Fontana, WI. 
130. Nørskov, J.K., et al., Origin of the Overpotential for Oxygen Reduction at a Fuel-Cell Cathode. The Journal of Physical Chemistry B, 2004. 108(46): p. 17886-17892.

131. Chevrier, V.L., et al., Hybrid density functional calculations of redox potentials and formation energies of transition metal compounds. Physical Review B, 2010. 82(7): p. 075122.

132. Lee, Y.-L., et al., Ab initio energetics of LaBO_\{3\}(001) (B=Mn, Fe, Co, and Ni) for solid oxide fuel cell cathodes. Physical Review B, 2009. 80(22): p. 224101.

133. HSC Chemistry(R) 6.0, 2002, Outokumpu.

134. Peter, B. and G. Franz, Potfit: effective potentials from ab initio data. Modelling and Simulation in Materials Science and Engineering, 2007. 15(3): p. 295.

135. Fumi, F.G. and M.P. Tosi, Ionic sizes and born repulsive parameters in the NaCl-type alkali halides-I: The Huggins-Mayer and Pauling forms. Journal of Physics and Chemistry of Solids, 1964. 25(1): p. 31-43.

136. Lantelme, F. and P. Turq, Ionic dynamics in the LiCl-- $\mathrm{KCl}$ system at liquid state. The Journal of Chemical Physics, 1982. 77(6): p. 3177-3187. 